\title{
LIBRO DE RESÚMENES \\ IV LATIN AMERICAN CONFERENCE ON RESIDENCY EDUCATION \\ LACRE 2019
}

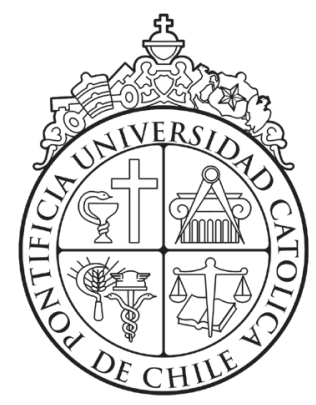

29, 30 y 31 de Mayo de 2019

Santiago de Chile 


\title{
Congreso Latinoamericano de Educación de Residentes: LACRE 2019 Latin American Conference on Residency Education: LACRE 2019
}

\author{
Trinidad Hoyl ${ }^{1}$, Arnoldo Riquelme², Linda Snell ${ }^{3}$.
}

\begin{abstract}
Profesor Asociado, Departamento de Medicina Interna, Directora de Postgrado Escuela de Medicina, Co-Chair Congreso LACRE1,3; Profesor Titular Departamento de Gastroenterología, Centro de Educación Médica y Ciencias de la Salud (CEMCIS), Director Departamento de Ciencias de la Salud, Comité Científico Congreso LACRE2; Pontificia Universidad Católica de Chile 1,2 ; Profesor de Medicina, Centro de Educación Médica, Universidad de McGill, Educadora Clínica Senior para CanMEDS en Royal College of Physicians and Surgeons de Canadá; Royal College of Physicians and Surgeons de Canadá, Universidad de McGill3.
\end{abstract}

Autor de correspondencia :mthoyl@uc.cl

Desde su primera versión en 2013, el Congreso Latinoamericano de Educación de Residentes (Latin American Conference on Residency Education: LACRE) ha tenido un gran impacto en la educación médica de postgrado en Chile y Latinoamérica, contribuyendo a aportar herramientas concretas, innovadoras y fácilmente replicables para la docencia de especialistas.

EI Congreso LACRE es organizado en forma bianual, por la Escuela de Medicina de la Pontificia Universidad Católica de Chile en conjunto con el Royal College of Physicians and Surgeons of Canada, y cuenta con expositores Canadienses y Latinoamericanos de reconocimiento y liderazgo internacional.

El Congreso LACRE se caracteriza por ser un espacio de encuentro entre docentes clínicos, jefes de programas de especialidades médicas y de ciencias de la salud, educadores, directores de postgrado, residentes, investigadores en educación médica y todos aquellos actores interesados en mejorar la educación de postgrado de especialistas en Chile y el mundo. En este Congreso se comparten ideas y soluciones a problemas comunes, lo que promueve le mejora continua en los programas de residencia, y así impacta positivamente en el mejor funcionamiento de los servicios de salud y centros formadores para cumplir con el fin último, que es la atención de los pacientes.

La principal motivación del primer congreso LACRE en el año 2013, fue el gran desarrollo de los programas de especialidades médicas desde los inicios del siglo XXI, con el advenimiento de nuevas tecnologías y la necesidad de mayor conocimiento y entrenamiento en el manejo específico de enfermedades complejas. El desarrollo de estos programas se basa en la capacidad de transmisión de conocimientos, destrezas clínicas relacionadas con el cuidado del paciente incluyendo procedimientos y actitudes profesionales de parte de especialistas altamente calificados en el manejo de pacientes, pero que por lo general, no tienen una formación profesional en los aspectos relacionados con la docencia. La capacitación docente requerida en este desarrollo del postgrado incluye un amplio espectro de actividades que las instituciones usan para apoyar a los facultativos en el adecuado desempeño de roles relacionados con la enseñanza, investigación y administración. En este contexto, los aspectos distintivos de la formación de especialistas y sub-especialistas suelen ser suplidos con las capacidades individuales del docente, pero en la medida que los programas crecen en complejidad y número de residentes, para cumplir con la alta demanda de especialistas en países en desarrollo y en áreas falentes, surge la necesidad de implementar sistemas de capacitación docente y acreditación de programas, que aseguren el adecuado funcionamiento de los mismos y que a su vez ofrezcan profesionales con todas las competencias necesarias para el ejercicio de la especialidad.

A nivel internacional existen varias iniciativas relacionadas con la docencia de postgrado y probablemente la conferencia internacional en educación de residentes (International Conference on Residency Education - ICRE) es el congreso con más prestigio a nivel mundial y es organizado por el Royal College of Physicians and Surgeons of Canada. A nivel latinoamericano no existían iniciativas a nivel de postgrado por lo que, por la Escuela de Medicina de la Pontificia Universidad Católica de Chile, el Royal College of Physicians and Surgeons of Canada, y la Organización Panamericana de Salud (OPS) conformaron un comité de trabajo para la organización del primer Congreso LACRE el año 2013. Luego de un largo proceso de más de 2 años, se logró plasmar este sueño, con el valioso patrocinio de la Sociedad Chilena de Educación en Ciencias de la Salud (SOEDUCSA), el Ministerio de Salud de Chile (MINSAL), la Agencia acreditadora de los programas universitarios de especialidades médicas (APICE-Chile) y la Asociación de Facultades de Medicina 
de Chile (ASOFAMECH). Ese primer congreso contó con más de 400 asistentes provenientes de 17 países, y se presentaron alrededor de 50 trabajos libres en sesión de posters.

En las versiones 2015 y 2017, el congreso mantuvo sus lineamientos generales, con una estructura participativa y potenciando la Educación Basada en Competencias, entorno a los roles CanMEDS. La versión 2017 del congreso LACRE reunió a 433 participantes, entre residentes de diferentes programas de especialidades médicas, y académicos de 14 países de la región. En ese LACRE se presentaron, luego de la plenaria Inaugural inolvidable dictada por Yvonne Steinert, experta mundial en el área de capacitación docente/Faculty development, 8 simposios, 21 talleres interactivos, y 30 trabajos orales además de 107 posters. Destacamos la primera versión del ICE Summit en español, iniciativa de la International Clinician Educator (ICE) Network, en la cual participan educadores clínicos de los 5 continentes, con reuniones en los principales congresos en educación médica del mundo, y que por primera vez se realizó en español, en el contexto del LACRE, co-dirigido por la Dra. Linda Snell del Royal College of Physicians and Surgeons of Canada, y de la Universidad de McGill, y el Dr. Arnoldo Riquelme, de la Facultad de Medicina de la Pontificia Universidad Católica de Chile.

Para el Congreso LACRE 2019, el tema central planteado es la discusión de soluciones innovadoras en la formación de residentes. El programa es variado y muy motivador, siguiendo el formato basado en simposios, y talleres que promueven el aprendizaje activo de los asistentes. El día pre-congreso, ofrece 4 sesiones paralelas durante la mañana, donde los participantes podían elegir entre áreas muy diversas: Cómo aprender el uso de la Simulación para la docencia de residentes; Liderazgo de Excelencia en Educación de residentes; Uso de las redes sociales en docencia; y Cómo introducir la investigación en su programa de residencia.

En la tarde, además se suma: ICE Summit, que en su segunda versión en español Controversias en Educación de Residentes: ¿sirve el examen final de la especialidad?, sin buen modelaje no existe nada. Sección para Residentes: donde se discuten temas tan interesantes como ¿Cómo desarrollar estrategias para mantener mi bienestar durante la residencia?, Uso de atención plena (mindfullness) en la relación clínica; promoviendo la docencia en una realidad donde el tiempo es escaso; Cómo integrar Social Media a la residencia; Cómo aprender a enfrentar la muerte: lo que nunca me enseñaron; como escribir artículos científicos durante la residencia.

El Congreso LACRE 2019 se inaugura con la conferencia plenaria "coaching para el éxito: traduciendo las enseñanzas de la música y los deportes a la educación de residentes". Durante los 2 días del Congreso se desarrollan 6 simposios, 23 talleres interactivos, 4 rondas de debate. En esta versión docentes líderes y residentes motivados por la educación médica de postgrado, presentan 25 trabajos libres orales y 75 posters, de temas innovadores que cubren áreas relacionadas con el ambiente educacional, diseños curriculares basados en competencias, modelos de enseñanza y aprendizaje al lado de la cama del paciente y en ambientes simulados, programas de entrenamientos procedimentales y evaluación de conocimientos y destrezas clínicas, cubriendo de buena manera, la mayoría de los ámbitos de la educación médica de postgrado.

Esperamos que esta cuarta versión del Congreso LACRE cumpla con las expectativas de los asistentes, y el Comité Organizador confía en que esta instancia de encuentro a nivel nacional y latinoamericano, se consolide en el tiempo como un foro para las Américas constituyéndose en un espacio para el desarrollo de la educación de postgrado. En el futuro, es nuestro deseo que el Congreso LACRE promueva la expansión a otras profesiones de la Salud, que cuenten con programas de formación avanzada de postgrado y que se convierta en un polo de desarrollo y comunicación de resultados de proyectos de investigación en educación de postgrado.

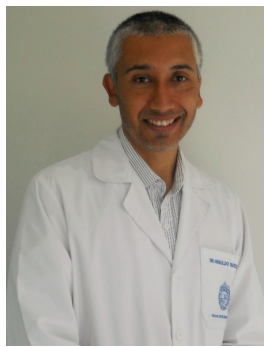

Arnoldo Riquelme Pérez, MD

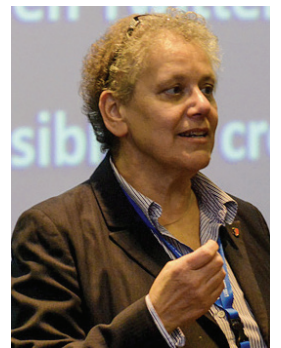

Linda Snell MD MHPE FRCPC FACP

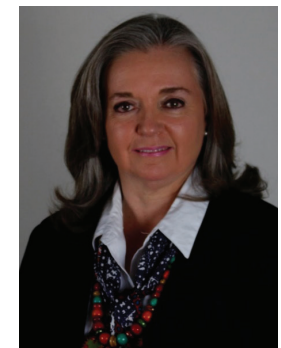

M Trinidad Hoyl MD

ARS MEDICA Revista de Ciencias Médicas

ISSN: 0719-1855 @ Dirección de Extensión y Educación Continua, Escuela de Medicina, Pontificia Universidad Católica de Chile. http://arsmedica.cl 


\section{Análisis político de la implementación del posgrado nacional de medicina familiar en el Ecuador}

Diaz Rodrigo (1)

(1) Pontificia Universidad Catolica del Ecuador

Introducción: El momento político histórico es importante para la medicina familiar en Ecuador. En 10 años (2001-2010) se formaron 201 médicos familiares, la necesidad en Ecuador es de 5200, en 2017 se graduaron 454 médicos familiares en una promoción, actualmente existen 900 médicos familiares en Ecuador. En 1987 se inicia etapa de formación de la especialidad y el posgrado, tiene lógica local. Se consolida una "escuela académica" de formación, de ecuador, médicos familiares trabajando en lo privado En 2008 inicia etapa de expansión y posicionamiento nacional, con propuesta publica a nivel nacional, en 2011 se construye el Posgrado de Medicina Familiar a nivel nacional e inicia en 2013, con 8 Universidades y una malla curricular única.

Metodo: Análisis de las dimensiones políticas del proceso de implementación de la especialidad Politics, relaciones políticas, interacciones Polity, instituciones políticas, contexto institucional, estructuras, reglas, instituciones: Policies. Políticas públicas, decisión final, resultados.

Resultados: Especialidad reconocida por el ministerio de salud - Programas de posgrado aprobados y en funcionamiento hasta el 2019 por el CES. Primera promoción graduada con 456 especialistas trabajando en el MSP, segunda promoción en formación 300 residentes. Nuevos médicos familiares implementando el Modelo de atención integral de Salud. Nueva cohorte (tercera) de 500 postgradistas.

Conclusiones: Es necesario: - Formar médicos familiares con competencias de liderazgo y formación política, esto implica un currículo diseñado con racionalidad interpretativa. - Se necesita Médicos Familiares trabajando en la Academia, y en posiciones técnicas de decisión en el Ministerio de Salud. - Hay que posicionar a la especialidad como la encargada de aplicar el modelo de atención. - Inculcar en los médicos familiares una actitud propositiva y proactiva para construir algo nuevo.

\section{Transferencia de habilidades artroscópicas en residentes desde el simulador al pabellón: un estudio prospectivo}

Ledermann Gerardo(1), Irarrazaval Sebastián (1), Besa Pablo(1), Rodrigo Andrés(1)

(1)Pontificia Universidad Católica de Chile, Santiago, Chile.

Introducción: Los programas de entrenamiento artroscópico para residentes han demostrado utilidad en la adquisición de habilidades quirúrgicas. Existe escasa evidencia sobre la capacidad de transferencia de estas habilidades al escenario real en pabellón. El objetivo de este estudio fue evaluar la capacidad de adquisición de habilidades artroscópicas en residentes, a través de un modelo de simulación de meniscectomía parcial (MPA), y su transferencia al escenario quirúrgico real. Material y método 11 residentes de Traumatología de primer y segundo año fueron capacitados en un programa de entrenamiento para MPA, consistiendo en una evaluación inicial en pabellón, 10 sesiones de entrenamiento en el modelo Sawbones® y una evaluación final en pabellón.

Métodos: Los procedimientos fueron evaluados utilizando como herramienta la "Arthroscopic Surgical Skill Evaluation Tool" (ASSET). Se utilizó como comparación la MPA realizada por 3 cirujanos expertos. Finalmente se evaluó la capacidad de retención de habilidades en los residentes después de 6 meses sin entrenamiento. El análisis estadístico de desempeño y capacidad de transferencia fue realizado mediante test de Wilcoxon y Mann Whitney. Significancia <0,05.

Resultados: Todos los residentes tuvieron una mejoría significativa en su desempeño durante el entrenamiento en el simulador (10 vs $39, \mathrm{p}<0,001)$, obteniendo los mismos puntajes finales que los expertos $(39, p<0,001)$. Los resultados obtenidos en pabellón pre y post entrenamiento mejoraron significativamente ( $14 \mathrm{vs} 36, \mathrm{p}<0,001$ ). Hubo una discreta diferencia entre los resultados obtenidos por los residentes post entrenamiento y la evaluación en pabellón (39 vs $36, p=0,01$ ), así como entre la evaluación en pabellón de los residentes post entrenamiento y cirujanos expertos (36 vs 39 , $p=0,01)$. No existieron diferencias en los resultados obtenidos al término del entrenamiento y los resultados obtenidos 6 meses posteriores a éste (39 vs 38, $p=0,165$ ). 
Conclusión: Este modelo de simulación de MPA mejora las habilidades quirúrgicas en residentes de Traumatología, las cuales son capaces de transferir al escenario quirúrgico. A su vez, los residentes entrenados son capaces de retener las habilidades adquiridas hasta al menos 6 meses posterior a su entrenamiento.

periódicos, así como la definición de funciones docentes específicas para los residentes. Existen algunas limitaciones importantes a considerar, destacando el limitado número de residentes que accede al programa, la necesidad de capacitar a los tutores y los recursos necesarios. Conclusión: Este proyecto busca ser una primera aproximación al problema identificado. A futuro, se espera que nuevas iniciativas contribuyan a una solución definitiva.

\section{Diagnóstico situacional de programas de residencia médica en la República de Panamá 2017-2018}

\author{
Noriega Lorena(1), Gutiérrez Raquel(2), Esquivel Maria(2) \\ (1) Caja de Seguro Social, (2)Universidad de Panamá
}

Introducción: La experiencia de Panamá en la formación reglada de médicos especialistas, tiene más de 60 años. Esta actividad se ha realizado con programas académicos vigentes, respaldados actividades docentes/asistenciales. En este proceso, participan únicamente hospitales estatales del Ministerio de Salud y de la Caja de Seguro Social. Cada hospital docente, ha implementado sus programas académicos sin procesos de actualización de contenidos programáticos permanentes y con escasa supervisión universitaria. La necesidad de actualización de los programas vigentes y de apertura de programas únicos universitarios de especialidades y sub-especialidades no oficiales, propició la creación de un espacio para la evaluación sistemática de los servicios y ambientes de aprendizaje formadores.

Metodología: Se instala Comisión Ad Hoc: 1 representante de Coordinación de Maestrías Clínicas, Universidad de Panamá, Curriculista especialista y una funcionaria especialista y docente de institución formadora. Se diseño un "formulario de reportes" que contiene 9 temas, referenciales de: Guía para la Acreditación de Residencias Médicas del Ministerio de Salud de Argentina, Postgraduate Medical Education WFME Global Standards y Consejo Americano para la Educación Medica Graduada, ACGME. Los servicios estuvieron representados por: coordinador docente, jefe de servicio, jefe de residentes y presidente de sociedad médica correspondiente. El coordinador de docencia de cada especialidad presentó la información solicitada.
Resultados: Acción con buena acogida por las instalaciones de salud. El espacio generado propició comunicación e intercambio efectivo entre funcionarios de hospitales docentes. Además asesoría y mediación por la comisión en puntos de conflicto. Presentaron satisfactoriamente el formulario de reportes los representantes de 23 servicios de los 6 programas universitarios vigentes y 58 servicios de especialidades no básicas y sub-especialidades de 10 hospitales. Se identificó diferencias entre servicios en cuanto a las 5 morbilidades mas frecuentes, procedimientos quirúrgicos, paridad tutores/aprendices, ambientes de aprendizaje, exposición a niveles de complejidad, rotaciones clínicas etc. Pocos servicios tienen líneas de investigación y manifestaron limitaciones para desarrollar investigación básica por falta de protocolos y ausencia de bases de datos confiables. La mayor parte de los servicios no ha instituido formalmente el portafolio o bítacora como archivo de evidencias producto de la formación.

Conclusiones: Se desarrolló con éxito acción comunicativa, evaluación sistemática y reflexiva como paso preparatorio para la actualización y acreditación de 50 programas de postgrado médico de 81 servicios formadores de hospitales docentes Se potenció la integración de los servicios de hospitales docentes, sociedades médicas y Universidad de Panamá. Se detectó diferencias en la formación que es necesario abordar con miras a obtener un estándar básico de formación por especialidad. De igual forma si se quiere que los residentes desarrollen competencias en investigación hay que crear las condiciones desarrollando bases de datos fiables, instaurando protocolos, facilitando asesoría y el acceso a los comités de bioética.

\section{Análisis de la formación interdisciplinaria del Cirujano Maxilofacial en un modelo curricular de residencia médica en el Hospital Clínico UC}

Rosa Andres (1); Ramírez Hernán (1); Goñi Ignacio (1); Vargas Alex (1)

(1) Pontificia Universidad Católica de Chile, Santiago, Chile.

Introducción: Tradicionalmente en Chile y gran parte del mundo, la Cirugía Máxilofacial (CMF) ha derivado de la formación odontológica de pregrado. Su desarrollo proviene de las intervenciones menores en el territorio oral y se ha constituido una especialidad quirúrgica que se practica en el tratamiento de diversas y complejas patologías maxilofaciales, muchas de ellas de resolución propia de la especialidad y varias otras de abordaje conjunto con especialidades médicas afines. En este contexto, se exige de la educación de postgrado en CMF adicionalmente al entrenamiento 
quirúrgico, que desde lo formativo permita la adecuada vinculación del profesional odontólogo al contexto médico, y desde el ámbito legal habilite y respalde el actuar profesional. Ante este gran desafío, muchos países han optado por la doble titulación médico - dental, sin embargo, en Chile no existe aquella disposición. El objetivo de este trabajo entonces es analizar la formación interdisciplinaria del especialista en CMF que provee un programa de formación de postítulo creado el 2014 en la Universidad Católica, único basado en el sistema de residencia médica con dedicación exclusiva y financiado que actualmente se imparte en Chile para esta especialidad.

Metodología: Metodología cualitativa. Se realizó un análisis documental utilizando como fuente de datos la documentación institucional relativa a organización departamental, programas de especialidad, registro de interconsultas y registro de pabellones de la Departamento de Cirugía Oncológica y Maxilofacial de la Pontificia Universidad Católica de Chile desde el año 2014 al 2017. Mediante análisis de contenido se identificaron instancias de interacción del residente de CMF con residentes de otras especialidades médicas y se realizó la indización por asignación y descripción sustancial en formato tabla.

Resultados: Se identificó y estableció un índice que incluye 1) Filiación departamental conjunta: CMF y Cirugía Cabeza y Cuello (CCYC) 2) Rotaciones clínicas propias de residentes de CMF: CCYC, Anatomía Patológica (AP), Anestesiología (A), Medicina interna (MI), Urgenciología (U), Otorrinolaringología (ORL), Cirugía Plástica $(\mathrm{CP})$, Radioterapia (RT), Oncología Médica (OM), Paciente crítico (UPC). 3) Rotaciones de otras especialidades médicas en CMF: CCYC 4) Especialidades médicas que han solicitado interconsultas a residentes de CMF: CCYC, A, MI, U, ORL, CP, OM, UPC, Ginecología $(G)$, Hematología (H), Endocrinología (E), Traumatología (T), Oftalmología (O), Pediatría (P), Cardiología (C), Gastroenterología (GA) y 5) Especialidades médicas que han programado pabellones en conjunto con residentes de CMF: CCYC, ORL, Cirugía Pediátrica (CPE), CP. Luego descripción y análisis por ítem.

Conclusiones: Se identifican múltiples instancias de interacción entre el residente de CMF y sus pares médicos residentes de diversas especialidades, tanto en contexto ambulatorio como quirúrgico, en la resolución de la patología propia y en actuar conjunto. Existe una interdependencia natural entre la Cirugía Maxilofacial y la Cirugía de Cabeza y Cuello. La formación interdisciplinaria enriquece la formación médica, diversifica el actuar intrahospitalario y fortalece el quehacer propio del residente de CMF. El tratamiento interdisciplinario y conjunto de la patología quirúrgica compleja permite mitigar el escaso soporte legal del odontólogo especialista en CMF.

\section{Valoración académica de las guardias por parte de residentes de psiquiatria: experiencia en Red UC-Christus y Hospital Clinic, Barcelona.}

Aedo Alberto(1), Anmella Gerard(2), Nitsche María Pía(1), Sagué María(2), Verdolini Norma (2), Calderón Jorge (1), Pacchiarotti Isabella(2), Murru Andrea (2)

(1) Pontificia Universidad Católica de Chile, Santiago, Chile. (2)Hospital Clínic Barcelona, España.

Introducción: En la actualidad, la formación de psiquiatras se realiza según diferentes currículos basados en competencias, los cuales consideran un desarrollo multidimensional que permitan una formación de calidad. Un espacio clínico para la adquisición de aprendizajes son las guardias en los servicios de urgencia, las cuales suponen un apoyo asistencial y también una instancia docente para el desarrollo de toma de decisiones en tiempo real. Sin embargo, a la fecha, existe escasa evidencia respecto a la utilidad que tienen en términos de aprendizaje para los residentes. Objetivo: Evaluar la percepción de utilidad que tienen las guardias de urgencia en la formación de los residentes de psiquiatría.

Metodología: Se realizó un estudio transversal tipo encuesta, a residentes de psiquiatría adultos de la Pontificia Universidad Católica de Chile, Chile (PUC) y del Hospital Clínic de Barcelona, (HCB). Se elaboró un cuestionario autoadministrado que incluyó aspectos sociodemográficos (edad, género, año de residencia) y utilidad de los turnos puntuados mediante escala Likert (0 a 10) en cuanto a: exploración psicopatológica, orientación diagnóstica, diagnóstico diferencial, prescripción de psicofármacos (antidepresivos, ansiolíticos, antipsicóticos, litio, estabilizadores del ánimo) y manejo de situaciones específicas (riesgo suicida, heteroagresividad, agitación psicomotora y comorbilidad médica). Usando la misma escala, se incluyó una pregunta sobre el rol del especialista del turno en la adquisición de conocimientos/competencias. Asimismo, se valoró el peso global de las guardias en la totalidad de la formación del residente mediante una pregunta de respuesta en franjas porcentuales $(0-25 \%, 25-50 \%, 50-75 \%$ y $75-100 \%)$.

Resultados: Completaron la encuesta un total de 28 residentes $(50 \%$ PUC, 50\% HCB). El 50\% eran mujeres, con una edad media de 28,6 \pm 2.34 años. El $35.7 \%$ cursaba primer año de residencia, repartiéndose equitativamente el resto entre segundo, tercer y cuarto año $(21.4 \%$ cada uno). En cuanto a la utilidad de los turnos (0-10 puntos), se obtuvo: Exploración psicopatológica ( $x=6.18 ; \mathrm{DE}=2.04)$; Orientación diagnóstica ( $x=6.75 ; \mathrm{DE}=1.92)$; Diagnóstico diferencial $(x=6.39$; $\mathrm{DE}=2.17)$; Manejo de: Riesgo suicida ( $\mathrm{x}=8.68 ; \mathrm{DE}=1.27)$, heteroagre- 
sividad ( $x=8.71 ; D E=1.18)$, agitación psicomotora ( $x=8.86 ; D E=1.14)$, paciente complejo ( $x=6.25 ; \mathrm{DE}=2.4)$; prescripción de: Antidepresivos $(x=3.57 ; \mathrm{DE}=2.46)$, ansiolíticos ( $\mathrm{x}=6.21 ; \mathrm{DE}=1.99)$, antipsicóticos $(x=6.07 ; D E=2.1)$, litio $(x=1,89 ; D E=2.09)$, estabilizadores del ánimo $(x=2.04 ; D E=2.2)$. Respecto al rol del especialista en la adquisición de conocimientos/competencias: $\mathrm{PUC}(\mathrm{x}=7.14 ; \mathrm{DE}=2.14) ; \mathrm{HCB}(\mathrm{x}=5.79$; $D E=2.64)$. En cuanto a la utilidad de las guardias respecto la totalidad de la formación, el $46.7 \%$ la ponderó del $25-50 \%$ y el $35.7 \%$ del $50-75 \%$. No hubo diferencias estadísticamente significativas entre hospitales en ninguna de las variables evaluadas.

Conclusiones: Los residentes de psiquiatría atribuyen a los turnos una proporción importante de la totalidad de su formación. Destaca el impacto docente que les supone en exploración psicopatológica, orientación diagnóstica, diagnóstico diferencial y manejo de situaciones específicas (paciente complejo, riesgo suicida, heteroagresividad y agitación), así como en la prescripción de ansiolíticos y antipsicóticos.

\section{Percepcion de la Incorporación de Residentes de Psiquiatría del Niño y del Adolescente en el Sistema de Atencion Primaria de Salud en Chile}

Aedo Karina (1), Coelho-Medeiros Elisa (1), Ramírez Verónica (1), Bedregal Paula (1)

(1) Pontificia Universidad Católica de Chile, Santiago, Chile

Introducción: Experiencias a nivel colaborativo entre Atención Primaria de Salud (APS) y especialistas en salud mental son evaluadas positivamente por el equipo de salud de APS, sin embargo, para psiquiatría infanto-juvenil no existe evidencia nacional al respecto.

Objetivo: Describir la percepción del equipo de salud dedicado a la atención de niños, niñas y adolescentes (NNA) sobre la incorporación de residentes de psiquiatría infanto-juvenil en el centro de atención Áncora-UC Madre Teresa de Calcuta de Puente Alto, Chile.

Metodología: Se realizaron dos grupos focales (de 10 personas cada uno, miembros del equipo de salud que trabajan con NNA) Las entrevistas se grabaron y transcribieron de manera literal. Dos investigadores realizan un proceso de reducción de datos, codificación y organización en categorías, basados en la Teoría de Calidad de A.Donabedian. Los resultados se expresan como frecuencias de mención en la discusión.
Resultados: En la temática de "beneficios", aparecen 12 categorías, resultando las más frecuentes el "apoyo clínico en evaluación, diagnóstico, manejo y seguimiento, adecuado al contexto de APS". En "dificultades" se identifican 12 categorías, donde la más frecuente es "no se logra trabajar con todos los estamentos no médicos". Por último, en "propuestas por mejorar", se construyen 15 categorías; la más frecuente es "aclarar el rol de los residentes como consultores y no como tratantes" Conclusiones: La información obtenida es relevante, considerando la necesidad de trabajo en equipo en los diversos niveles de atención de salud que requiere la formación de psiquiatras infantiles.

\section{Evaluación de la implementación de un curso de procedimientos para residentes}

Olascoaga Ana Cecilia, García Angélica, Mas Guiliana

(1) Universidad Peruana Cayetano Heredia, Lima, Perú.

Introducción: Los residentes de medicina interna y especialidades clínicas deben adquirir habilidades para realizar procedimientos en la práctica clínica. Tradicionalmente los residentes aprenden a realizar estos procedimientos mediante la práctica en servicio, al inicio observando a otros, y luego realizándolos directamente a los pacientes que atienden y con la supervisión y guía (o no) de sus tutores o de sus residentes mayores. Esta forma de aprendizaje es afectada por la oportunidad y por la capacidad del tutor o residente mayor que enseña. La seguridad del paciente debe ser preservada en el entrenamiento de los residentes. Se pretende describir la experiencia en la implementación de un Curso de Procedimientos para Residentes y la evaluación del mismo según los niveles 1 y 2 del modelo de evaluación de programas educacionales de Kirkpatrick.

Metodología: Se implementó un curso teórico práctico para capacitar a los residentes en la realización de procedimientos diagnósticos y terapéuticos (punción arterial, punción lumbar, intubación endotraqueal, cateterismo venoso central, paracentesis, colocación de sonda nasogástrica). El abordaje teórico se hizo a través de un espacio virtual y el abordaje práctico utilizando simulación con fantomas en el Centro de Simulación. Se organizaron grupos de 5 a 6 residentes asignados a un tutor. Se programó una práctica de 2 a 3 horas por cada procedimiento. El objetivo fue aplicar lo aprendido en las lecturas y videos y recibir retroalimentación. Participaron 15 docentes. El curso se evaluó mediante el método de evaluación de programas de Kirkpatrick. El nivel Kirkpatrick I - Reacción se evaluó mediante encuestas de satisfacción por módulo, con seis preguntas cerradas y una pregunta cualitativa abierta que solicita la opinión libre del alumno sobre el curso; estas respuestas se 
analizaron mediante el método de Teoría Fundamentada. El nivel Kirkpatrick II - Aprendizaje se evaluó mediante exámenes escritos online y durante las prácticas de simulación de cada procedimiento mediante las listas de cotejo.

Resultado: 60 residentes de primer año de medicina interna y especialidades clínicas desarrollaron el curso. Kirkpatrick I - Reacción: El 100\% de los residentes consideró que los objetivos del curso se cumplieron, que los materiales y las prácticas facilitaron su aprendizaje. El análisis cualitativo de las opiniones libres de los residentes encontró opiniones positivas sobre la metodología del curso y las prácticas de simulación, destacando la percepción de haber aprendido y el sentimiento de confianza para realizar los procedimientos. Kirkpatrick II - Aprendizaje: Los exámenes escritos, que fueron 8 , uno por cada procedimiento y que entre todos sumaron 100 puntos, tuvieron como nota promedio 87.5 (mínima 77.5, máxima 97.5). Las prácticas de simulación de cada procedimiento se evaluaron mediante las listas de cotejo, todos los residentes aprobaron, con notas promedio entre 18.6 Y 19.8 sobre 20 puntos.

Conclusiones: El curso de procedimientos fue factible de realizar, logró las competencias deseadas y fue muy bien valorado por los alumnos. Debe continuar realizándose con las mejoras propuestas para los residentes de todas las sedes. La experiencia se puede replicar en otras especialidades con distintos procedimientos.

\section{Diseño y evaluación de un simulador de traqueostomía percutánea de bajo costo. Un estudio piloto.}

Kattan Eduardo, Bravo Sebastian, Putz Francisca, Corvetto Marcia, De La Fuente Rene, Vera Magdalena

(1) Pontificia Universidad Católica de Chile Santiago, Chile.

Introducción: La traqueostomía percutánea (TP) es un procedimiento frecuente realizado por los intensivistas(1). Se asocia hasta con un 5-10\% de complicaciones(2), concentrado en los operadores no expertos y en la fase de aprendizaje(3). En la literatura no se han reportado protocolos de entrenamientos comprehensivos, y los simuladores comerciales disponibles presentan elevado costo y baja fidelidad. El objetivo fue describir el diseño de un simulador de bajo costo de TP, y evaluar su fidelidad y validez de constructo

Metodología: Se diseñó un simulador de bajo costo con implementos obtenidos en una ferretería, tráqueas bovinas obtenidas ex vivo, piel sintética y una cámara endoscópica conectada a un teléfono móvil. Se probó el simulador en 5 expertos y 5 novatos, quienes recibieron material instruccional previamente. Se aplicó una encuesta de satisfacción a los participantes, y se evaluó la validez de constructo en base a: tiempo total de procedimiento, éxito de primera punción, puntaje en escala global de apreciación(OSATS), distancia total recorrida por las manos y movimientos totales realizados, parámetros derivados de sensores Imperial College Surgical Assist Device(ICSAD). Se presentan datos como mediana[rango intercuartil] y porcentajes. Se utilizaron pruebas no paramétricas para la comparación entre grupos(Test $U$ de Mann Whitney y Test exacto de Fisher) y correlación de Spearman para la concordancia inter-observador. Se consideró $p<0.05$ como estadísticamente significativo.

Resultados: Se construyó exitosamente el simulador, con un costo fijo de $\$ 19.000$ pesos y uno variable de $\$ 3200$ por uso. Presentó alta satisfacción usuaria, con fidelidad elevada tanto del procedimiento global como de sus subprocesos. La evaluación por expertos presentó buena concordancia inter-observador (correlación de Spearman $r 2=0.81$ ). Los expertos realizaron un procedimiento más fluido (puntaje OSATS 8,5[6.5-15] vs 24.5[23-25] $p=0.02$ ), en menor tiempo (545 vs 297 s p =0.02), con mayor economía de movimientos (distancia recorrida 113[99-130] vs 77[69-86] metros $p=$ 0.02 , movimientos totales 569 [506-691] vs 441 [388-481], $p=0.02$ ) y mayor éxito al primer intento de punción ( $100 \%$ vs $20 \%, p=0,048)$.

Conclusiones: Se describe el diseño exitoso de un simulador de bajo costo para TP, con alta fidelidad, satisfacción usuaria y buena validez de constructo, permitiendo discriminar entre expertos y novatos. Considerando su bajo costo y facilidad de ensamblaje, puede ser implementado en diferentes centros del país y Latinoamérica. A futuro, permitirá desarrollar un programa de entrenamiento de habilidades en residentes de medicina intensiva, para lograr la adquisición de competencias sin poner en riesgo la seguridad de los pacientes.

\section{Desarrollo de material para familiares de pacientes, potenciando las competencias CanMeds. Experiencia: Acompañándote: Integrando a los niños en los Cuidados Paliativos}

Pavlovic Andres (1), Claro Juan Carlos (1), Valles Isabel (2), Traeger Sophie (3)

(1) Pontificia Universidad Católica de Chile, (2)Centro del Cáncer UC, Santiago, Chile. (3)Jungle Studio

Introducción La educación médica por competencias CanMeds está continuamente buscando nuevas herramientas para el desarrollo de las habilidades descritas como intrínsecas (liderazgo, 
colaborador, promotor de la salud, académico) 1, con el objetivo de una formación integral centrada en el paciente2. Frecuentemente el cuidado de pacientes en la atención de cuidados paliativos implica una sobrecarga física y emocional para su entorno familiar3, situación que tiende a producir menor integración de los niños a la experiencia de enfermedad del familiar4,5. Dado estos antecedentes, un equipo multidisciplinario (residente, staff, diseñadora gráfica y psico-oncólogas infantiles), desarrollaron un libro para ofrecer un recurso a la familia del paciente en cuidados paliativos, centrado en los niños y sus cuidadores, que eduque respecto al proceso de enfermedad, favorezca la expresión de emociones y permita desarrollar recursos para el afrontamiento de esta experiencia.

Metodología El material desarrollado consiste en un cuento narrado por dos personajes nietos pequeños de una paciente en cuidados paliativos. En el transcurso de la historia el lector (niño/a) tendrá la posibilidad de interactuar con el material (dibujando, enumerando, etc.), además de reflexionar en torno a distintas temáticas. Además, cuenta con un cuadernillo para el cuidador, donde se presentan sugerencias para facilitar el acompañamiento del niño/a durante este proceso y el abordaje de cada uno de los temas expuestos en el libro. La confección del material se basa en la técnica narrativa, donde por medio de la estimulación de la imaginación, se sugieren cambios y se ofrece una toma gradual de conciencia ante la situación de enfermedad acontecida en el sistema familiar. La elaboración de un material educativo y de reflexión como este tuvo varios aspectos que incidieron de forma directa en el desarrollo del residente. Por un lado, el hecho de dirigirse a una comunidad tradicionalmente sub-representada en los cuidados paliativos como son los familiares más jóvenes de los pacientes en fases avanzadas de una enfermedad terminal subraya el rol de promotor de la salud. Por otro lado, el residente pudo desarrollar herramientas de coordinación, facilitando las diversas reuniones entre los distintos miembros del equipo durante todo el proceso de creación del material, y asumiendo un rol de liderazgo en la relación de financiamiento, promoción y difusión que se establece con la red de salud UC-Christus.

Discusión/Resultado: La experiencia de desarrollo de material informativo para niños con familiares en cuidados paliativos, evidenció el desarrollo de múltiples competencias CanMeds. Entre ellas el rol de comunicador, colaborador, liderazgo, promotor de la salud. Dichas competencias deben ser desarrolladas en forma constante ante los complejos desafíos que se proponen en el desarrollo de la medicina actual, donde la relación médico-paciente y médico familia deben ser pilares del tratamiento integral.

Conclusión: El Desarrollo de material educativo para familiares como herramienta de crecimiento de las competencias Canmeds ofrece múltiples beneficios tanto para el residente como para el equipo multidisciplinario que lo compone, además del paciente y sus familiares. Dichas experiencias deben ser evaluadas como herramientas válidas para el potenciamiento de competencias CanMeds en el futuro.

\section{Proceso de Selección para el Ingreso al Sistema único del internado médico en la República de Panamá durante los años, 2017 al 2018.}

Susan Beitia(1), Monica Rodriguez(2), Oris de Calvo(3)

(1) Ministerio de Salud, (2)Caja de Seguro Social, (3)Universidad Nacional de Panama

Introducción: En la República de Panamá, la Comisión Nacional de Docencia de Médicos Residentes e Internos, es el organismo de carácter científico responsable de, organizar y planificar los procesos académicos, de capacitación y certificación de todos los médicos internos. Creado mediante RESOLUCIÓN PRESIDENCIAL N.786 14 DE AGOSTO DEL 2014 y formado por representantes del Ministerio de Salud (MINSA), Caja de Seguro Social (CSS), Facultad de Medicina de la Universidad de Panamá, Colegio Médico, y la Federación Nacional de Médicos Residentes e Internos (FENAMERI). Este organismo trabaja enmarcado en las regulaciones legales que rigen el ingreso al internado en base al puntaje obtenido en el examen de certificación básica. Decreto Ejecutivo No. 57 (De martes 28 de Marzo de 2017) En Panamá, son obligatorios dos años de internado médico para obtener la idoneidad profesional. Objetivo: 1. Organizar el Proceso de ingreso al Sistema único del internado médico.

Métodos: 1. Los graduados, después del 2014, de la Carrera de Medicina de universidades nacionales y extranjeras, realizan un examen de certificación de conocimientos generales de la práctica médica. 2. El Consejo Interinstitucional de certificación básica de medicina, establece el puntaje mínimo para la aprobación del examen. 3. La Comisión Nacional de Docencia elabora la lista de los aspirantes que aprobaron el examen, en orden decreciente, de acuerdo al puntaje obtenido en el examen de certificación. 4. Los hospitales estatales, MINSA y CSS, indican el número de plazas disponibles para el internado. 5. En una sesión, a la cual acuden, todos los aspirantes que aprobaron el examen, cada uno de ellos, elige el hospital para el primero y segundo año de internado (Viva Voz). 6. Los aspirantes graduados antes del 2014, se les adjudica la plaza de acuerdo al año de graduación de la carrera. 7. El primer año se rota en los Servicios de Hospi- 
tales de tercer nivel: Ginecología y Obstetricia, Cirugía General, Urgencias Médicas, Medicina Interna, Pediatría y Psiquiatría. El segundo año, en todo el territorio Nacional, con énfasis en Atención Primaria. 8. Al finalizar los dos años, la Universidad de Panamá otorga el título: Postgrado de Especialización en Medicina Clínica.

\section{Resultados:}

\begin{tabular}{l|c|c|c|c}
\hline & 2017* & Porcentaje & $\mathbf{2 0 1 8}$ & Porcentaje \\
\hline $\begin{array}{l}\text { Aspirantes al examen } \\
\text { de Certificación }\end{array}$ & 438 & 100 & 669 & 100 \\
\hline Aprobaron el examen & 303 & 69.2 & 451 & 67.4 \\
\hline Plazas ofertadas & 190 & 100 & 451 & 100 \\
\hline Aspirantes a plazas & 187 & 98.4 & 446 & 98.9 \\
\hline
\end{tabular}

*En octubre 2017, inicia la regulación del ingreso al internado.

Conclusiones: 1. El sistema único de internado ha permitido la distribución de plazas de internado en base a los méritos de los aspirantes. 2. Se ha organizado, ordenado y planificado el ingreso y finalización del internado, lo que permite un mayor número de aspirantes a las plazas de residencias médicas.

\section{Efficacy of practical skills workshops during surgical hands-on training course}

Ignatavicius Povilas (1), Gudaityte Rita (1), Dambrauskas Zilvinas (1), Tamelis Algimantas (1)

\section{(1) Lithuanian University of Health Sciences}

Introduction: Practical surgical skills are an important part of the career of surgical residents and young practicing surgeons. We, therefore, developed and in 2016 started intensive two-day surgical hands-on training course.

Methodology: The course consists of theoretical (lectures and workshops on evidence-based medicine) and practical (workshops on open and laparoscopic surgery, sonography for trauma, ultrasound guided procedures) parts. Thirty-two international participants were enrolled in the course in 2016 and 2017. After the course all participants were asked to fill the evaluation form consisting of 14 questions. All questions could be rated from 1 (Unsatisfactory) to 5 (Excellent). Evaluation forms were assessed by the authors.
Results: In the second year the total length of the course increased from 12 to 16 hours. There was a 2.3 -fold increase in the overall length of the practical skills part (from 33\% to 75\%). The evaluation score increased in 12 questions from 14. The highest increase was observed in the evaluation of the course expectations - the score increased from 4.0 to 4.9. The total evaluation score increased from 4.4 in 2016 to 4.8 in 2017.

Conclusions: In the second-year surgical hands-on training course was evaluated better than in the first year. In all 14 questions the evaluation score exceeded 4.5 and reached almost excellent level. We believe that these changes were determined by the increased total length of the course and especially by significantly increased length of the practical skills workshops. To meet the needs and expectations of the participants of surgical hands-on training courses, the biggest part of such course should be dedicated to practical skills workshops. Theoretical part should consist of short, clear and specific lectures.

\section{Prevalencia del síndrome de desgaste laboral (Síndrome de Burnout) en residentes de una clínica de 4 nivel de complejidad en la ciudad de Cali.}

Durán Carlos (1), Salazar Natalia (1), Cañas Alexa (1), Salazar Agatha (1), Tavera Geraldine (1)

\section{(1) Universidad Icesi}

Introducción: El síndrome de desgaste laboral (Burnout) hace referencia a un tipo de estrés laboral generado en profesionales que mantienen una relación constante y directa con otras personas. Se sabe que tiene prevalencias elevadas en profesionales del área de la salud y que además de alterar la calidad de vida del profesional, produce un efecto negativo en la calidad de la prestación de servicios de salud. El cuestionario Maslach es el instrumento más utilizado en todo el mundo para su medición y consta de 22 ítems que miden 3 aspectos del síndrome: Cansancio emocional, despersonalización, realización personal. Objetivo: Determinar la prevalencia del síndrome de Burnout en los residentes de las diferentes especialidades médicas de una clínica de 4 nivel de la ciudad de Cali, Colombia. Metodología: Estudio descriptivo transversal, mediante encuesta autodiligenciada para determinar variables sociodemográficas, se incluyeron: año de residencia, especialidad, número de horas semanales laborales. El análisis estadístico fue univariado y bivariado para observar la distribución de las variables y determinar si existía asociación entre ellas. El análisis estadístico se realizó con el programa estadístico Epistat v.3.0 y Epilnfo v. 6.0. Se consideró una $\mathrm{p}<0.05$ como significativa. 
Resultados: Se evaluó a 62 residentes, excluyendo a aquellos que se encontraban en vacaciones o que estaban realizando prácticas en otras instituciones. 17 residentes de pediatría (27\%), 28 de medicina interna (45\%), 7 de radiología (11\%), 6 de ginecología (10\%), 2 de psiquiatría (3\%) y 2 de dermatología (3\%), que fueron evaluados durante el segundo semestre del 2018. Los estratos socioeconómicos predominantes fueron los estratos 4 y 5 con $39 \%$ y $34 \%$. En cuanto a estado civil de los encuestados, 45 (79\%) son solteros. La mayoría están en primer año con 22 (35\%). El promedio de número de horas presenciales fue de 65,1 horas a la semana. De los tres componentes evaluados, el 63\% de la muestra presenta puntajes altos en el componente de desgaste emocional, lo cual esta descrito en otros estudios similares. Existe variabilidad entre el predominio de los componentes por especialidad, lo cual es esperable dadas las condiciones individuales de cada una. Se encontró síndrome de burnout en 7 residentes (11\%): 3 hombres y 4 mujeres. Las variables sociodemográficas entre las personas con desgaste laboral fueron similar al resto de la muestra. No se encontraron residentes de primer año que presentaran síndrome de burnout y el promedio de horas laborales/semana reportadas por los residentes con síndrome de desgaste laboral fue mayor al promedio reportado por la muestra en total (70,8 Vs 64.3 horas).

Conclusiones: La prevalencia de los síntomas de Síndrome de Burnout en la población de residentes evaluada es similar a las reportadas en otros países. Es necesario implementar acciones activas para combatir el síndrome de Burnout y mejorar el equilibrio calidad de vida versus exceso de trabajo y la generación de políticas públicas que garanticen un sustento y mejor calidad de vida a los residentes.

\section{Evaluation of Progress and Competency of Surgery Residents after the Self-Directed Simulation-Based Basic Ultrasound Skills Course}

Dambrauskas Zilvinas (1) Gudaityte Rita (1), Ignatavicius Povilas (1), Tamelis Algimantas (1)

\section{(1) Lithuanian University of Health Sciences}

Introduction: HybridLab is a fusion of distance learning and medical simulation that allows learners to train $24 / 7$ at their work place without presence of the instructor and/or technician. Our previous studies showed a strong positive effect of this learning approach on enhancement of basic trauma management, basic open and laparoscopic skills among the residents of the surgical specialties. Basic ultrasound skills and mastering of extended focused assessment with sonography in trauma (eFAST) is important part of surgical training. Therefore, we evaluated the feasibility and effectiveness of the self-directed simulation-based ultrasound skills course. Objective: to evaluate the progress and the effectiveness of autonomous training of basic ultrasound skills and eFAST protocol among the junior surgery residents.

Methodology: Basic ultrasound skills and eFAST course was set-up using the HybridLab platform and the BodyWorks Eve high fidelity trainer (MedaPhor) in September, 2018. In total, 27 junior surgical residents of LUHS were enrolled into the study. In the first phase of the study we assessed the progress of the residents throughout the course consisting of 3 modules: ultrasound physics and the operation of machine, probe manipulation, and eFAST protocol. In the second phase of the study we will assess the competency of the residents to perform eFAST/obtain standard views on simulated patients using the 10-point scale (views in 5 standard probe positions (pleura, RUQ, pelvis, LUQ, subxiphoid) are rated 0 - cannot perform, 1 - needs assistance, 2 - performs independently). Using the sequence of 10 standardized scenarios and pre-recorded eFAST views we will evaluate the ability of the residents to identify the life-threatening signs, including pneumothorax, hemothorax, free fluid in the abdomen, cardiac tamponade (min score - 0, max score - 10 points).

Results: From the point of view of the residents, one of the limitations of the ultrasound trainer was that the theoretical lectures on ultrasound physics and operation of the ultrasound machine were not available online (in contrast to the other HLab skill courses, when all the lectures, reading material and tests are provided online in the virtual learning environment). However, all the course participants reviewed the provided teaching material (score 100\%) and on average spent 1.23 hours on this module. On average residents achieved a $90 \%$ progress score in hands-on probe manipulation training module and spent 1.02 hours for this task. Residents on average spent 35 minutes in the eFAST training module with progress score of $80 \%$.

Conclusions: HybridLab provides access to real patient-based training of basic ultrasound skills and eFAST protocol and allows residents to train independently at their own pace. High fidelity mannequin-based trainer engages students into the highly effective medical simulation. The time dedicated for the theory studying and hand-on practice is comparable or longer than traditional face-to-face courses. However, the clinical impact of this self-directed course will be known after further assessment of the residents on the simulated patients and testing employing the standardized scenarios with ultrasound views (currently undergoing) 
Implementación de Bitácora Electrónica de Registro de Actividades para Residentes de Anestesiología y Reanimación: Experiencia Ara-Udp en Monitorización de Progresión de Residentes de Primer Año

Flores Alejandro (1)

(1) Universidad Diego Portales, Santiago, Chile.

Introducción: El registro de los procedimientos realizados por parte de los residentes en formación es una herramienta importante en la objetivación y monitoreo continuo del proceso educativo, adquiriendo especial relevancia cuando los programas de formación, dado su naturaleza teórico-práctica, requieren de rotaciones especificas en centros formadores distintos al centro principal. Objetivo: Describir los resultados de la implementación de una bitácora de registro electrónico de actividades por parte de los residentes del programa de postítulo de Anestesiología y Reanimación de la Universidad Diego Portales de primer año y evaluar esta intervención como una herramienta de gestión de la formación continua de los residentes, con el fin de permitir un análisis por parte de la dirección del programa y la eventual intervención en caso de detectar incumplimientos en los estándares mínimos definidos.

Resultados: De un total de 19 residentes que cursan su primer año de formación, se obtienen datos de 17 de ellos ( 2 de ellos suspenden transitoriamente el programa), los que realizaron un total de 22.287 registros de actividades supervisadas en sus primeros 6 meses de rotación, con un promedio de 1.311 registros de actividades supervisadas por tutor, por residente (218 registros de actividades mensuales). 4209 actividades registradas (19\%) corresponden a pacientes con clasificación ASA 1, 14974 (67\%) a clasificación ASA 2, 2755 actividades (12\%) en pacientes clasificados como ASA 3, 333 (2\%) a ASA 4 y 18 (0\%) a ASA 5. Se constata un porcentaje de éxito de las actividades registradas como procedimientos de un $97,1 \%$. Durante los primeros 6 meses de programa se constata el cumplimiento de un $72 \%$ de los procedimientos establecidos por las agencias certificadoras de la especialidad.

Conclusiones: La migración de los programas de formación hacia un enfoque basado en competencias, requiere demostrar que las destrezas procedurales se han alcanzado. El registro electrónico es una herramienta promisoria para este objetivo, ofreciendo la posibilidad de analizar la información de manera eficiente, para el apoyo docente continuo.

\section{¿Qué priorizan los médicos recién graduados al momento de elegir la institución para hacer la residencia?}

Facioni Clara (1), Eymann Alfredo (1), Sanchez Mariana (1), Reboiras Fabiana (1), Faingold Diego (1), Figari Marcelo (1)

(1) Instituto Universitario del Hospital Italiano de Buenos Aires, Argentina.

Introducción: Los motivos por los cuales los profesionales que se presentan a rendir examen para el ingreso a las residencias médicas escogen una u otra institución, no han sido muy explorados hasta el momento. Los actuales aspirantes para las residencias en salud se caracterizan por pertenecer a una generación signada por grandes transformaciones culturales. La elección de espacios o instituciones flexibles y poco estructuradas caracterizan en buena medida a los jóvenes que hoy ingresan al mundo laboral. Nos proponemos explorar cuáles son las motivaciones de los aspirantes en la elección de la institución para realizar la residencia.

Métodos: Metodología utilizada Se realizó una encuesta anónima, voluntaria y presencial luego de finalizado el examen de conocimientos de selección múltiple a los aspirantes a las residencias médicas. El instrumento contó con preguntas cerradas (edad, sexo, universidad de procedencia, especialidad elegida), y una pregunta abierta sobre las tres condiciones que consideran más importantes para elegir una institución en orden de importancia.

Resultados: De un total de 1139 aspirantes que rindieron el examen de conocimientos de selección múltiple para ingresar a las residencias médicas se completaron 1113 encuestas. El 59\% de los aspirantes fueron mujeres. Los aspirantes provenían de la Universidad de Buenos Aires (46,9\%), Universidades Nacionales (26,5\%), Privadas (16,3\%) y Extranjeras (10,2\%). Se inscribieron para especialidades quirúrgicas $(44,7 \%)$, clínicas $(49,2 \%)$ y otras $(6,1 \%)$. Las respuestas se categorizaron en las siguientes dimensiones: calidad académica, prestigio institucional, caudal y complejidad de pacientes, clima laboral, condiciones laborales, equipamiento y tecnología, investigación, trato hacia los pacientes, trabajo en equipo, desarrollo profesional, localización, acreditación y otras. Las primeras condiciones mencionadas al momento de elegir la institución fueron calidad académica $(65,4 \%)$, prestigio institucional (16\%) y caudal y complejidad de pacientes (5,9\%). Las segundas fueron clima laboral $(21,6 \%)$, calidad académica (20\%) y prestigio institucional (18,8\%). En tercer lugar, mencionaron clima laboral $(16,1 \%)$, condiciones laborales $(16,1 \%)$ y calidad académica $(14,5 \%)$. No se encontró asociación entre las condiciones elegidas y sexo, universidad de origen y especialidad elegida. 
Conclusiones: Los aspirantes a la residencia valoran en primer lugar características relacionadas a su formación. Por otra parte, es relevante la importancia adjudicada al clima y las condiciones laborales.

\section{Desafíos de la jefatura de residentes: dificultades y logros del desempeño del rol en un hospital universitario de comunidad}

Facioni Clara (1), Eymann Alfredo (1), Sanchez Mariana (1), Reboiras Fabiana (1), Nardi Maria (1), Vazquez Lucia (1), Carrió Silvia (1), Figari Marcelo (1)

(1) Instituto Universitario del Hospital Italiano de Buenos Aires, Argentina

Introducción: El jefe de residentes es un profesional que ha finalizado recientemente su formación en una especialidad. Es una figura muy valorada que se percibe como un reconocimiento dentro del sistema de salud y tiene un rol estratégico en el liderazgo de procesos de innovación en los programas formativos. El jefe de residente es el principal referente para orientar los procesos de aprendizaje e integrar el grupo de residentes. Por otra parte, es una figura estratégica de articulación entre la residencia, los responsables del programa y los profesionales de planta. Por lo tanto este estudio se propone caracterizar las actividades desarrolladas por los jefes de residentes e identificar los principales logros y dificultades percibidos en el ejercicio del rol en un hospital universitario de comunidad.

Metodología: Se realizó una encuesta electrónica confidencial y autoadministrada a todos los jefes de residentes al finalizar su jefatura. Se definieron las variables edad, sexo, especialidad, número residentes, otros profesionales con roles docentes, respaldo por responsables del programa e institucionales, porcentaje de tiempo para actividades (docentes, gestión académica, asistencial, investigación y administrativas), situaciones más y menos gratificantes percibidas, necesidad de capacitación y recomendación para ser jefe de residentes.

Resultados: Contestaron la encuesta $37 / 42$ jefes de residentes, $46 \%$ fueron mujeres y su edad $31 \pm 3,1$ años. El 43,4\% pertenecía a una residencia clínica, 33,3\% quirúrgica y $23,3 \%$ a otras especialidades. La relación de jefe de residentes por residente fue 1/ 12,7 (37/471) y de otros profesionales con roles docentes por residente fue 1/4 (118/471). El 84\% percibió respaldo por los responsables del programa (director, coordinador, supervisor) y $49 \%$ por el Comité de Residentes y Becarios. La dedicación a actividades fue: asistenciales $26,1 \%$, gestión académica $24,8 \%$, docentes $23,6 \%$, administrativas $15,9 \%$ e investigación $9,6 \%$. Las situaciones percibidas como más gratificantes fueron categorizadas como desarrollo del rol docente, reconocimiento de la tarea y trabajo en equipo. Las situaciones percibidas como menos gratificantes fueron categorizadas como relaciones interpersonales, realización de tareas administrativas, dificultades para la gestión y sobrecarga asistencial. El 56,8\% reconoce falta de capacitación. Las necesidades de capacitación que informaron fueron categorizadas como manejo de grupo, liderazgo y formación docente. El 94,3\% recomendaría realizar la jefatura de residentes, y los principales motivos categorizados fueron: el crecimiento profesional, el desarrollo de un rol docente y el manejo de grupo.

Conclusiones: Los jefes de residentes refieren elevada carga de tareas administrativas y baja en investigación. La mayoría de las situaciones más y menos gratificantes estuvieron vinculadas con las relaciones interpersonales. La mayoría reconoce falta de capacitación para la tarea pero valora la experiencia y la recomendaría a un compañero.

\section{Programa de Bienestar de los Residentes: Orientación y Evaluación Inicial}

\author{
Cordero Mary Ana (1), González Marisela (1), De la Rosa Cynthia (1) \\ (1) Escuela de Medicina y Ciencias de la Salud Tecnológico de Monterrey, México
}

Introducción: El propósito del Programa de Bienestar para Residentes de la Escuela de Medicina del Tecnológico de Monterrey, primero de su tipo en México, es mejorar el bienestar de los residentes, educándolos sobre el bienestar y su relación con el profesionalismo. Se implementó una sesión específica de orientación sobre el programa de Bienestar y evaluación inicial para los médicos residentes de primer año.

Métodos: En marzo de 2018, como programa piloto, se diseñó una sesión de orientación para el Programa de Bienestar el cual se ofreció a 75 médicos residentes que ingresaron a los 16 programas de especialidades médicas en el Programa Multicéntrico de Residencias Médicas de la Escuela de Medicina y Ciencias de la Salud del Tecnológico de Monterrey y de la Dirección Regional de Servicios de Salud. La sesión de 3 horas se dividió en cuatro secciones: introducción al Programa de Bienestar, consejería para residentes médicos, evaluación básica de salud mental y programa de prevención de abuso de sustancias. Para la evaluación básica de salud mental, con consentimiento previo, se aplicaron 3 pruebas individualmente: inventario de Ansiedad de Beck, inventario de Depresión de Beck y pruebas proyectivas.

Resultados y conclusiones: Después de completar la orientación, a los residentes se les ofreció programar una reunión de asesoramiento individual con el psicólogo de la escuela para completar la evaluación 
inicial de salud mental y hablar sobre sus preocupaciones sobre el bienestar profesional, emocional, físico y social. Ellos propondrían un plan de trabajo y programarán reuniones de seguimiento. Si la situación lo justifica, el consejero tendrá la capacidad de remitir al residente al departamento de apoyo correspondiente.

\section{Programa de Bienestar de Residentes: Desarrollo Docente para Mentores}

Cordero Mary Ana (1), Macías Mariano (1), Vargas Gabriel (1), Uribe Leslie (1)

(1) Escuela de Medicina y Ciencias de la Salud Tecnológico de Monterrey, México.

Introducción: El propósito del Programa de Bienestar para Residentes de la Escuela de Medicina del Tecnológico de Monterrey, primero en México, es implementar una iniciativa de calidad para mejorar el bienestar de los residentes, aumentar su compromiso, educarlos sobre el bienestar y su relación con el profesionalismo. La capacitación para mentores que participarán en el programa de bienestar es fundamental para la implementación del programa.

Métodos: Como piloto, 27 Profesores Médicos del Programa de Pediatría se diseñó e implementó la capacitación para ser mentores del programa de bienestar para médicos residentes a partir de 2018. Se requirió la acreditación de dicha capacitación que se divide en tres módulos: (a) introducción al programa de bienestar, (b) estrategias para bienestar, salud mental y prevención del suicidio, y (c) mentoría y servicios de apoyo para referencia de casos.

Resultados y conclusiones: El primer módulo se llevó a cabo en noviembre de 2017, con la asistencia de 27 mentores durante el cual se dio la introducción al programa, los objetivos y la discusión de video-casos. El segundo módulo se llevó a cabo en enero de 2018 e incluyó el entrenamiento QPR (Question, Persuade \& Refer) para la prevención del suicidio. El tercer módulo, se centró en técnicas de entrevista y la identificación de casos que deben remitirse a servicios de apoyo, mediante una capacitación de simulación de casos en febrero de 2018. Después de completar su capacitación, los mentores deben tener reuniones individuales con los residentes en las que hablarían sobre las preocupaciones actuales, así como identificar cualquier problema o inquietud en cada una de las categorías, presentar un plan de trabajo y programar una reunión de seguimiento dentro de tres meses. Si es necesario, el mentor puede remitir al residente al departamento de apoyo correspondiente.

\section{Programa de mentoreo para médicos residentes: diseño e implementación}

\author{
Cordero Mary Ana (1), Valencia Oscar (1), Dávila José (1), De la \\ Rosa Cynthia (1)
}

(1) Escuela de Medicina y Ciencias de la Salud Tecnológico de Monterrey, México.

Introducción: En el Modelo educativo Tec 21 del Tecnológico de Monterrey se ha declarado el mentoreo como componente principal. El consejo regulador nacional (CONACYT), a través de los Programas Nacionales de Posgrado de Calidad (PNPC) ha declarado en el Marco de Referencia para la Evaluación y Seguimiento de Programas de Especialidades Médicas que los programas deben contar con Tutoría Académica. En el Programa Multicéntrico de Especialidades Médicas del Tecnológico de Monterrey, los médicos residentes reciben dicho acompañamiento de manera intermitente, irregular e informal, por profesores que asumen libremente el rol de mentor, sin la intervención de la Institución. Por lo tanto, se requiere un programa sólido de Mentoría, de acompañamiento personal y académico, que cuente con procesos que influyan positivamente en el desempeño académico, laboral y de satisfacción personal del residente; y que a su vez sea un sistema formal de evaluación del proceso de acompañamiento al alumno y cuente con indicadores precisos.

Métodos: El diseño inició en abril 2018 y concluyó en julio 2018. Se seleccionaron para la implementación inicial los programas de Calidad de la Atención Clínica, Cardiología, Geriatría, Neonatología y Radiología basado en que son las Especialidades Médicas que se someterán en junio 2019 al proceso de reacreditación por el CONACYT-PNPC. El programa de mentoreo tiene la finalidad de potenciar el rendimiento académico, la satisfacción personal y la proyección laboral de cada uno de los residentes. Los residentes participarán en la selección de su mentor (excepto los R1 a los cuales se les asignará un mentor) indicando tres opciones en orden de preferencia. Una vez realizada la asignación, se realizarán al semestre 2 entrevistas con su mentor, con la documentación correspondiente.

Resultados y conclusiones: Se implementó el programa en 4 de las 5 especialidades médicas, previa capacitación de los mentores. Se realizará una revisión inicial de la implementación y acciones de mejora en enero 2019. Posteriormente se implementará progresivamente mentoría formal en la totalidad de los programas (16). 


\section{Programa de mentoreo: capacitación para mentores de médicos residentes de especialidades médicas}

Mary Ana Cordero(1), Oscar Valencia(1), Minerva Cardona(1), Cynthia De la Rosa(1)

\section{(1) Escuela de Medicina y Ciencias de la Salud Tecnológico de Monterrey, México.}

Introducción: En el Modelo Tec 21 del Tecnológico de Monterrey se ha declarado la importancia del mentoreo como eje central. El Consejo nacional regulador de los Programas Nacionales de Posgrado de Calidad (CONACYT-PNPC) ha declarado en el Marco de Referencia para la Evaluación y Seguimiento de Programas de Especialidades Médicas que los programas deben contar con Tutoría Académica. En el Programa Multicéntrico de Especialidades Médicas del Tecnológico de Monterrey se requiere un programa sólido de Mentoreo, de acompañamiento personal y académico, que cuente con procesos que influyan positivamente en el desempeño académico, laboral y de satisfacción personal del residente; así como, de un sistema formal de evaluación del proceso de acompañamiento al alumno. La capacitación para mentores que participarán en el programa es fundamental para la implementación.

Métodos: Se seleccionaron para iniciar la capacitación de mentores los programas de Calidad de la Atención Clínica, Cardiología, Geriatría, Neonatología y Radiología, programas de especialidad médica que se someterán en junio 2019 al proceso de reacreditación por el CONACYT-PNPC. La capacitación básica para mentores consistió en tres sesiones de 2 horas cada una. En la primera se realizó una exposición corta del programa y un taller de análisis del rol del mentor a través de casos. En la segunda sesión se impartió un Taller QPR (Question, Persuade, Refer) para la prevención del suicidio, identificación y referencia de los alumnos los servicios de apoyo. La tercera sesión se desarrolló como un taller de simulación de casos, en el que estudiantes de medicina fungieron como "supuestos" residentes; los profesores se dividieron en equipos de 3 a 4 personas, cada uno con un rol asignado ( 1 entrevistador y el resto de observadores) rotando en 6 estaciones, el objetivo de esta sesión era orientar a los profesores en cuanto al manejo de problemáticas comunes de los residentes.

Resultados y conclusiones: Se capacitó a los mentores de los programas seleccionados, quienes realizarán la implementación en 2018 y revisión de resultados en enero 2019. Posteriormente se emprenderá la capacitación de mentores para implementar progresivamente mentoreo formal en la totalidad de los programas de especialidad médica.

\section{Autoestima en Médicos Residentes de un Hospital Escuela de Asunción}

Gonzalez Fatima (1), Camacho Cinthia (2), Pavón Juana (2)

(1) Facultad de Ciencias Médicas- UNA, (2)Universidad Nacional de Asunción

Introducción: El periodo de Residencia Médica está marcado por una sobrecarga tanto de horario como de responsabilidades, ya que los mismos constituyen en gran medida la estructura de atención principal de las varias instituciones sanitarias. Los recursos humanos de un sistema de salud son vitales para el buen funcionamiento de este, por lo que el trabajo constante en la autovaloración permite un mejor cuidado del profesional y por ende una mejor calidad de servicio. La autoestima o autovaloración es un factor fundamental para el buen o mal desempeño en los médicos, así como la determinación del nivel de autoestima que de ser necesario puede mejorar con diversas técnicas. Se busca de esta manera evaluar el sentimiento de autovaloración que tienen los residentes del Hospital de Clínicas, Facultad de Ciencias Médicas de la Universidad Nacional de Asunción. Metodología: Estudio descriptivo, de corte transverso, tamaño de muestra es 61 Médicos Residentes del Hospital de Clínicas de la FCM-UNA, en quienes se aplicó la Escala de autoestima de Rosemberg que consta de diez preguntas de valoración personal y evalúa el sentimiento de satisfacción, clasificando los resultados en tres niveles según la sumatoria de puntos: • Autoestima baja (0 a 25) • Autoestima Normal (26 a 29) • Autoestima alta (30 a 40). Resultados: Al analizar los niveles de autoestima se determinó que de 61 Médicos Residentes 13\% (8 residentes) presentan Autoestima Baja, 41\% (25 residentes) Autoestima Normal y un 46\% (28 residentes) Autoestima Alta. En cuanto a los factores que determinaron los niveles de Autoestima Alta se observó con mayor frecuencias que en su mayoría los residentes estaban muy de acuerdo con el ítem: Siento que soy una persona digna de aprecio y el ítem: Soy capaz de hacer las cosas bien como la mayoría y muy en desacuerdo con el ítem: A veces pienso que no soy bueno en nada, con respecto a tener una actitud positiva hacia uno mismo/a se observó que una mayoría estaba muy de acuerdo y un menor número estaban de acuerdo. Entre los factores determinantes de los niveles de Autoestima Baja a pesar de estar de acuerdo con los ítems: siento que soy una persona digna de aprecio y tengo actitud positiva hacia mí mismo/a, se observó una disminución en cuanto a la puntuación en los ítem: siento que no tengo muchos motivos para sentirme orgulloso/a de mí, a veces me siento verdaderamente inútil y a veces pienso que no soy bueno/a para nada, que resultaron en una baja puntuación. Conclusión: el sentimiento de satisfacción que tienen consigo mismo los Médicos Residentes fue en mayor porcentaje de autoestima elevada, y en menor porcentaje autoestima Baja. 


\section{Taller para directores de programas de especialidades médicas: diseño e implementación con colaboración internacional}

Mary Ana Cordero (1), José Dávila (1), Carlos Félix (1), Cynthia De la Rosa (1)

(1)Escuela de Medicina y Ciencias de la Salud Tecnológico de Monterrey, México.

Introducción: Para lograr un gran programa de residencia es indispensable que se trabaje en el diseño de éste abarcando temas como los principios de enseñanza, evaluación, así como estrategias que favorezcan el bienestar y rendimiento académico de los residentes. Por lo tanto, se diseñó e implementó un taller para directores de los programas de especialidades médicas del Programa Multicéntrico del Tecnológico de Monterrey en colaboración con el Royal College of Physicians and Surgeons of Canada. Metodología: Se estableció una colaboración con el Royal College of Physicians and Surgeons of Canada y se diseñó el taller dirigido a los directores de programa y directores adjuntos de residencias médicas de las diferentes instituciones que conforman el programa de residencias Multicéntrico (Tec Salud, Hospital Metropolitano, Hospital Regional Materno Infantil, Hospital Psiquiátrico de Saltillo y Hospital CIMA-Chihuahua). El taller consistió en una actividad previa y dos sesiones presenciales dirigidas por dos profesores internacionales. A través del trabajo grupal se revisaron los principales roles y responsabilidades, así como habilidades de liderazgo con las cuales debe contar un director de programa. De igual manera se capacitó a los profesores en cuanto al diseño de un sistema de evaluación y aprendizaje efectivo.

Resultados y conclusiones: En septiembre de 2018, se impartió el taller de dos días y 9 temas. Fue ofrecido a 39 directores de programa y directores adjuntos de las diferentes instituciones que conforman el programa de residencias Multicéntrico. Dos profesores internacionales fueron los encargados de guiar las sesiones en las cuales se abordaron problemáticas comunes dentro de un programa de residencias, estrategias para resolverlas y consolidar un gran programa de residencia médica. Al final se recibió retroalimentación positiva por parte de los asistentes.

\section{Taller de Liderazgo para jefes de residentes: una iniciativa innovadora e internacional}

Mary Ana Cordero (1), José Dávila (1), Carlos Félix (1) Glen Bandiera (2), Oscar Casiro (3), Marisela González (4), Fernanda Velázquez (1)
(1) Escuela de Medicina y Ciencias de la Salud Tecnológico de Monterrey, (2) University of Toronto, Royal College of Physicians and Surgeons of Canada, (3) Royal College of Physicians and Surgeons of Canada, (4) Oficina Regional de Servicios de Salud de Nuevo León

Introducción: Un rol de liderazgo responsable e integral debe ser desarrollado para los jefes de residentes durante su entrenamiento para permitirles contribuir y apoyar el desempeño académico de sus pares así como necesidades y recursos. Un taller de liderazgo específico para los jefes de residentes fue diseñado e implementado integrando métodos innovadores y una colaboración internacional con el Royal College of Physicians and Surgeons of Canada. El Programa Multicéntrico de Residencias Médicas de la Escuela de Medicina y Ciencias de la Salud del Tecnológico de Monterrey junto con la Oficina Regional de Servicios de Salud ofrecen 16 programas de especialidad médicas. Las áreas específicas de responsabilidad deben ser definidas y habilidades como la comunicación y la negociación deben ser desarrollados. Se realizó un taller de liderazgo específico para los jefes de residentes el cual fue diseñado e implementado integrando métodos innovadores con colaboración internacional del Royal College of Physicians and Surgeons of Canada. Métodos: Durante 2017, se estableció una colaboración con el Royal College of Physicians and Surgeons of Canada y el taller fue diseñado con 2 sesiones y 10 temas sobre liderazgo, funciones como jefe de residentes, habilidades de negociación, bienestar y servicios de apoyo. La metodología del taller fue presentación plenaria, trabajo individual y discusión grupal sobre los principales objetivos del jefe de residentes así como las responsabilidades, políticas, servicios de apoyo y recursos disponibles. Se implementaron ejercicios específicos y actividades de comunicación y desarrollo de habilidades de negociación. Resultados y conclusiones: En febrero de 2018 , se realizó un taller de dos días y diez temas el cual fue ofrecido a 31 residentes: 16 jefes de residentes y 15 subjefes en las instalaciones del Tecnológico de Monterrey. Un profesor internacional, 2 profesores nacionales y 4 directores de programas participaron como facilitadores y guiaron las sesiones. Al final se recibió retroalimentación positiva de los participantes de manera informal. Los siguientes pasos incluyen una reunión mensual de los jefes de residentes con el coordinador de las especialidades médicas para discutir y darle seguimiento a las necesidades, recursos y casos específicos que surjan. Los talleres futuros incluirán encuestas de calidad y un portafolio electrónico, una evaluación del desempeño del jefe de residentes durante el 2018, analizando el impacto del taller sobre el trabajo del jefe de residentes. 


\section{Docencia y satisfacción Laboral en un Hospital Escuela de Asunción}

\author{
Gonzalez Fatima (1), Camacho Cinthia (1)
}

(1) Universidad Nacional de Asunción

Introducción: La docencia constituye una tarea exigente que obliga al docente a capacitarse de manera constante, mantener un buen relacionamiento con pares y con alumnos, el docente debe estar bien preparado y tener una base de conocimiento sólida, abierto a la crítica y a la discusión. Este esfuerzo y dedicación muchas veces puede no ser reconocido ni valorado, lo que genera en el mismo situaciones de disconfort y estrés. Evaluamos con este trabajo la satisfacción personal, las dificultades laborales y el autoconocimiento, determinando los factores que influyen de mayor manera en estas áreas y que definirán en gran medida la calidad de enseñanza del docente. Metodología: Estudio descriptivo, corte transversal, utilizando como instrumento una encuesta compuesta por 25 items, validada en la institución con un grupo de 15 docentes (auxiliares docentes y escalafonados). Muestreo probabilístico por conveniencia. Resultados: Participaron 117 docentes de la Facultad de Ciencias Médicas de la Universidad Nacional de Asunción. 57 de ellos eran Docentes Escalafonados (48.7\%) y 60 (51.3\%) Auxiliares de la Docencia, las edades oscilaron entre 35 y 40 años en un 43.6\%(51 docentes) y más de 50 años en un 28.2\% (33 docentes) En el área de Satisfacción personal se detectaron más factores positivos que negativos, para lo 78 docentes $(66,7 \%)$ la distribución de tiempo y trabajo se encuentra equilibrada, el trabajo es valorado por los estudiantes según 93 docentes(79,5\%), consideran que el esfuerzo tiene resultados en el $89,7 \%$ (105 docentes) y en el $100 \%$ de los casos cuentan con apoyo y reconocimiento familiar, y disfrutan trabajando, considerando que el trabajo de Docencia es un Honor en el 64,1\% de los casos(75 docentes). En cuanto a la Dificultad laboral, 51,3\% de los docentes (60) considera que la dificultad con otros docentes disminuye la calidad de su trabajo, y 69,2\%(81) se siente mortificado de no poder ganar suficiente dinero como para mantenerse a largo plazo. la presión continua no afecta su estado de ánimo en el $64,1 \%$ (75 docentes) así como los conflictos con estudiantes en el $51,3 \%$ (60), se destaca además que en el 79,5\% de los casos (93 docentes) pueden realizar trabajos intersectoriales sin presentar ningún tipo de conflictos. Al referirnos al autoconocimiento, 108 docentes (92,3\%) sabe con precisión que se espera de su trabajo y convierten los obstáculos en experiencias positivas, 111 (94,9\%) refiere que le gustan los retos, a pesar de que eso signifique ir contra corriente, y el 100\% refiere invertir su tiempo en hacer cosas buenas. Conclusiones: el análisis de tres área que afectan directamente a las actividades docentes demuestran que existe una satisfacción personal traducido esto principalmente en el disfrute del ejercicio de la profesión, que las dificultades laborales están relacionadas con los propios colegas y con la remuneración recibida, y que, existe un conocimiento de lo que se espera y lo que se puede dar por medio de la docencia.

\section{Percepción del ambiente educativo por parte de los residentes, luego de la reapertura de una residencia de Gineco- obstetricia en Formosa, Argentina.}

\author{
Etchegaray Martin (1), Rosende Hugo (2); Casale Roberto (1)
}

(1) Federación Argentina de Sociedades de Ginecología y Obstetricia (FASGO) (2) Hospital de la Madre y el Niño - Formosa - Argentina

Introducción: La Residencia de Gineco-obstetricia del Hospital de la Madre y el Niño, es el único centro formador de especialistas en la provincia de Formosa, Argentina. Por diversos motivos, algunos relacionados con déficit en el ambiente educativo, se generó una falta de interés para formarse en dicho centro, no ingresando médicos entre 2011 y 2015. Por indicación del Ministerio de Desarrollo Humano se inició un trabajo de coaching organizacional para preparar a la institución a fin de adaptarla a los nuevos estándares educativos de Argentina y para generar una mejora del ambiente educativo. Desde 2016 volvieron a ingresar residentes de manera ininterrumpida hasta la fecha. Objetivo Evaluar la percepción del ambiente educativo que tienen los residentes de Gineco-obstetricia de la Residencia del Hospital de la Madre y el Niño de Formosa. Material y método Estudio observacional, descriptivo y transversal, utilizando como instrumento el cuestionario D-RECT en su versión traducida y validada al español. El mismo está constituido por 35 ítems agrupados en 9 subescalas, que son: atmósfera educativa, trabajo en equipo, rol del tutor de la especialidad, asesoramiento y entrenamiento por parte del tutor, educación formal, colaboración entre pares, adaptación del trabajo a la competencia de los residentes, accesibilidad a los supervisores y alta de pacientes. Las repuestas a cada afirmación planteada en cada dominio se clasifica utilizando una escala de Likert de 5 puntos, siendo el puntaje 1 totalmente en desacuerdo y el 5 totalmente de acuerdo; considerando que a mayor puntaje mejor percepción de clima educativo. Score máximo: 5. Las encuestas se realizaron "on line", utilizando la plataforma de formularios de encuestas de Google. La participación fue de tipo voluntaria, garantizando la confidencialidad de la información enviada. Los datos fueron recolectados desde el 31 
de agosto y hasta el 15 de septiembre de 2018. Resultados El $61.5 \%$ de los participantes fueron mujeres y el $38.5 \%$ hombres. El 76.9\% argentinos y el 23.1 extranjeros. Mediana de la edad 29 años (26-37). Residentes 1er año $(n=5) 38.5 \%$, residentes 2 do año $(n=5)$ $38.5 \%$, residentes 3er año $(n=2) 15.4 \%$ y jefa de residentes $(n=1) 7.5 \%$. Media de la percepción global del ambiente educativo: 3.91. Entre las dimensiones se destacan: trabajo en equipo 3.97 (DS 0.74), colaboración entre pares 4.10(DS 0.85), adaptación del trabajo a la competencia de los residentes 4.02 (DS 0.74), accesibilidad a los supervisores 4.2 (DS 0.69) y pases de guardia 4 (SD 0.89). Conclusiones El D-RECT ha demostrado ser un instrumento válido, confiable y factible para medir la calidad del clima de aprendizaje médico. El resultado positivo de la percepción del ambiente educativo en la residencia del Hospital de la Madre y el Niño permite concluir, en parte, los motivos por los que se sostiene el ingreso de residentes desde su reapertura.

\section{Modelo Simulado de Baja Complejidad dara Entrenamiento en Episiotomía}

Pérez Mario (1)

(1) Hospital Italiano de Buenos Aires

Introducción: El modelo simulado de entrenamiento ha demostrado ser una herramienta útil para la enseñanza de una destreza en médicos sin entrenamiento quirúrgico previo. El objetivo del entrenamiento en un modelo simulado es enseñar y evaluar a los especialistas en formación, para que puedan realizar el procedimiento sin poner en riesgo la seguridad de la paciente.

Métodos: El entrenamiento se realizó a los residentes de primer año en la especialidad de tocoginecología en dos etapas; primero con un modelo con esponjas, para conocimiento y manejo del instrumental y suturas, y en la segunda etapa, con lengua de vaca, ya que tes un tejido muscular con diferentes texturas de semimucosa En todas las sesiones se realizó una introducción teórica, una mostración, y luego de la actividad de entrenamiento, un debriefing con feedback constructivo. Las evaluaciones son estructuradas, con una herramienta diseñada para las destrezas quirúrgicas (OSATS) y realizadas por dos profesionales diferentes

Resultados: Luego del período de entrenamiento con un modelo simulado, se logra capacitar al profesional, para que pueda realizar la práctica en forma supervisada en la paciente

Conclusión: Las técnicas de simulación de baja complejidad, apoyadas en feedback constructivo y evaluaciones estructuradas, son una inmejorable herramienta de entrenamiento profesional, ya que los errores de la curva de aprendizaje no son corregidos sobre la paciente, mejorando la seguridad de la atención para la embarazada, en un momento vulnerable, como es el proceso de atención del parto.

\section{Determinación de los Factores de Riesgo Ergonómico en Aprendices Durante Curso de Formación Básica para Microcirugía}

Rojas Manuel (1)

\section{(1) Universidad de Antioquia}

Introducción: La microcirugía es una técnica quirúrgica que se fundamenta en la motricidad fina y precisión, razón por la cual el cirujano debe contemplar una serie de factores que afectan notablemente su desempeño, estas técnicas presentan un nivel de dificultad y complejidad debido a que el cirujano debe contar con unas competencias específicas para su ejercicio profesional, lo que plantea diferentes retos quien debe adaptarse a una serie de posturas estáticas prolongadas de cabeza, cuello, espalda y piernas, generando fatiga física, dolores y molestias, adicionalmente enfrentarse a factores como el cansancio visual, el movimiento continuo y repetitivo del instrumental, adaptación corporal al microscopio y al entorno de trabajo; durante las fases iniciales de formación es fundamental instruir al aprendiz con un manejo eficiente de la ergonomía mediante un seguimiento minucioso y objetivo a lo largo del desarrollo de los procedimientos programados, lo que pretende minimizar la generación toma posiciones erradas y forzadas que se pueden convertir en fatiga muscular, molestias, dolor, generando errores en la técnica; nuestro objetivo es determinar los factores de riesgo que están enfrentados los aprendices identificando la relación existente entre las malas posturas ergonómicas frente a la generación de dolencias físicas.

Métodos: se valoró el desempeño ergonómico de 71 participantes que incluía residentes de I a IV año, fellows, especialistas y cirujanos de mano pertenecientes al posgrado en Cirugía Ortopédica y Traumatología incluidos en el programa de formación básica para microcirugía. Quienes a lo largo de las actividades programadas inherentes al curso fueron parte de seguimiento para cada participante por los docentes de curso quienes, contaban con un formato creado a base de escalas en medición y registro de desempeño ergonómico, donde se identificó la presencia molestias relacionadas con errores posturales; lo que determinó la relación entre la posición ergonómica frente a la presencia de dolor. 
Resultados: se encontró mal desempeño ergonómico de los participantes en la posición de miembros inferiores y pies con un $54.8 \%$, desorganización del instrumental con un $45.8 \%$, posición incorrecta miembros superiores y espalda ambos con un $17 \%$, cabeza y cuello con el $5.6 \%$, al finalizar las actividades los participantes referenciaban molestias y dolor en región lumbar $25.3 \%$, trapecio $19.7 \%$, cuello $18.5 \%$, manos $14 \%$, otros $8.5 \%$.

Conclusiones: mediante el seguimiento de tipo observacional se logró identificar las zona de trapecio y lumbar como las regiones corporales más sensibles a presentar molestias y dolor durante el trabajo microquirúrgico como consecuencia a faltas ergonómicas evidenciadas en el presente estudio, errores en cabeza, cuello, brazos se trasfieren molestias en zona de hombros y espalda atribuidos a mal ajuste de la altura del cabezote del microscopio, silla; a su vez posiciones erradas de piernas y pies sensibilizan la zona lumbar; sin dejar de mencionar el mal manejo del instrumental incidiendo negativamente en el temblor manual, baja en la precisión y errores en la técnica.

\section{La evaluación promocional y final integradora de especialidades médicas en la Facultad de Ciencias Médicas-UNC}

Lucchese Marcela (1), Lizzio Salvador (1), Ceballos Irma (1), Vargas Alejandra (1), Güizzo María (1), Novella María (1)

\section{(1) Facultad de Ciencias Médicas- Universidad Nacional de Córdoba}

Introducción: En el marco de procesos institucionales y de acreditación de carreras de posgrado, la Facultad de Ciencias Médicas (FCM) - Universidad Nacional de Córdoba- realiza modificaciones en la modalidad de obtención del título en las especializaciones médicas. Implicó una nueva forma de comprender el sistema de evaluación, en términos de proceso y resultado, con una perspectiva integral, contextualizada y permanente. Las carreras que aplicaron este sistema, son aquellas que desde 2015 fueron acreditadas por CONEAU (Comisión Nacional de Evaluación y Acreditación Universitaria). El objetivo consiste en describir el proceso de evaluación promocional y final integradora de especialidades médicas en el período 2016 a julio 2018. Metodología: La perspectiva metodológica se conforma desde la investigación-acción que consiste en una postura exploratoria frente a cualesquiera definiciones iniciales de la propia situación e interpreta lo que ocurre desde los sujetos (Elliot, 1993) con la intención de revisar las prácticas evaluativas y mejorarlas (Carr y Kemmis 1988). Desde esta perspectiva se efectuó un trabajo organizado con consejos académicos de las especializaciones médicas, directores de centros formadores, equipo pedagógico de la FCM, veedores y estudiantes para identificar cambios en el sistema de evaluación, rol de los integrantes y condiciones para implementar la evaluación. Para el registro de la actividad de evaluación, se diseñó una ficha con ítems: Carrera, Sede del Examen, Centros Formadores, Fecha, Portfolio de los residentes, Trabajo científico, Tribunal examinador, Postulantes a rendir, espacio físico, observaciones y firma del veedor. Desde el sector administrativo de la FCM se efectuaron registros de los centros formadores, cantidad de alumnos y especializaciones en cada examen de los años 2016, 2017 y 2018 obtenidas del SIU GUARANI. Resultados: Se diseñó e implementó una herramienta de seguimiento común para las evaluaciones promocionales y finales bajo el formato de Portfolio. Durante año 2016, cohorte 2015 -Promoción a $2^{\circ}$ año- se presentaron 17 carreras: 7 básicas y 10 post-básicas. 77 centros formadores: 50 públicos y 27 privados. Durante el año 2017, cohorte 2015 -Promoción a $3^{\circ}$ año- se presentaron 16 carreras: 7 básicas y 9 post-básicas, 70 centros formadores: 45 públicos, 24 privados y uno con hospitales asociados. Durante el año 2017, cohorte 2016 -Promoción a $2^{\circ}$ año- se presentaron 18 carreras: 7 básicas y 11 post-básicas, 77 centros formadores: 46 públicos, uno con hospitales asociados y 30 privados. En cuanto a la cantidad de estudiantes, en la primera etapa del año 2018: para la cohorte 2015: 98 alumnos en condiciones de rendir el examen promocional a $4^{\circ}$ año y 95 alumnos en condiciones de rendir el examen final integrador. Cohorte 2016: 212 alumnos en condiciones de rendir el examen promocional a $3^{\circ}$ año. Cohorte 2017: 193 alumnos en condiciones de rendir el examen promocional a $2 \circ$ año. Asistieron como veedores: 12 profesionales: 5 representantes de la FCM, 1 coordinadora del Equipo Pedagógico y 6 pedagogas. Conclusiones: Las modificaciones del sistema de evaluación permitieron un seguimiento sistemático de los estudiantes año por año, un acuerdo de criterios de evaluación y articulación entre aspectos académicos y asistenciales.

\section{Acreditación en alta calidad de posgrados de Facultad de Medicina de la Universidad de Antioquia Medellín, Colombia 2018}

Toro Antonio (1)

(1) Universidad de Antioquia, Medellín, Colombia.

Introducción: Actualmente Colombia cuenta con 60 facultades de medicina, solamente 27 facultades son acreditadas en alta calidad. Dentro de estas 27 facultades se encuentra la facultad de medicina de la universidad de Antioquia. El consejo nacional de educación 
superior - CESU, aprobó en el acuerdo N 3 de 2016 los nuevos lineamientos para la acreditación de especialidades médicas. $\mathrm{Ha}$ sido una política de la facultad de medicina hacer una autoevaluación con miras a acreditación de sus posgrados de la facultad de medicina. Metodología La facultad de medicina cuenta con 45 posgrados, 4 maestría y un doctorado. Dentro del plan de acción de la administración actual de la facultad de medicina “Plan de acción 2017 - 2020", se estableció realizar autoevaluación de todos sus posgrados y postular para acreditación el $50 \%$ de sus posgrados acreditables hasta el 2020. Se fortaleció el comité de acreditación, se está implementando una cultura de acreditación en la facultad. Resultados: A noviembre de 2018 se han acreditado en alta calidad los siguientes posgrados: ANESTESIOLOGIA Y REANIMACIÓN ACREDITADO Resolución 9603 2013-07-25 Vigencia 6 años CX PLÁSTICA, MAXILOFACIAL Y DE LA MANO ACREDITADO Resolución 2533 2014-02-25 Vigencia 8 años CIRUGÍA GENERAL ACREDITADO Resolución 1156317 de julio de 2018 Vigencia 6 años DERMATOLOGIA ACREDITADO Resolución 15557 2013- nov 1 Vigencia 8 años GINECOLOGIA Y OBSTETRICIA ACREDITADO Resolución 9818 2013-07-31 Vigencia 6 años MEDICINA DEPORTIVA ACREDITADOREACREDITADO RESOLUCIÓN 05125 2018-23-03 VIGENCIA 6 AÑOS NEUROLOGIA ACREDITADO Resolución 9602 2013-07-25 Vigencia 6 años PEDIATRIA ACREDITADO Resolución 2532 2014-02-25 Vigencia 6 años TOXICOLOGÍA ACREDITADO Resolución 23757 22-12-2016 Vigencia 6 años Estamos a la espera de respuesta de CNA para el programa de psiquiatría quien ya realizó proceso de autoevaluación con mirad a acreditación y recibió visita de pares. Se enviaron informes de autoevaluación con miras a re acreditación los posgrados de anestesiología y reanimación y ginecología y obstetricia. Actualmente se encuentran realizando proceso de autoevaluación con miras a acreditación los posgrados de cirugía pediátrica, medicina física y rehabilitación, medicina de urgencias, nefrología pediátrica, medicina interna, alergología, maestría en epidemiología clínica, otorrinolaringología, oftalmología, patología, maestría en educación en salud. El programa de toxicología clínica recibió el premio a la calidad que concede la AUIP (Asociación Universitaria Iberoamericana de Postgrado) en marzo de 2018. Conclusiones: Se está consolidando la cultura de la autoevaluación en la facultad de medicina, logrando que se puedan acreditar programas a nivel nacional y en un futuro a nivel internacional para los residentes. Existen algunas limitaciones importantes a considerar, destacando el limitado número de residentes que accede al programa, la necesidad de capacitar a los tutores y los recursos necesarios. Conclusión: Este proyecto busca ser una primera aproximación al problema identificado. A futuro, se espera que nuevas iniciativas contribuyan a una solución definitiva.

\section{Bienestar residentes Facultad de Medicina, Universidad de Antioquia Medellín, Colombia 2018}

\author{
Caro Yuri (1), Toro Antonio (1), Duque Luis (1)
}

(1) Universidad de Antioquia, Medellín, Colombia.

Introducción: Cuando se compara las tasas de estrés, depresión, trastornos de ansiedad, ideación suicida, de los estudiantes de medicina con la población general, se encuentra mayor prevalencia de estos problemas. La oficina de bienestar de la facultad de medicina de la Universidad de Antioquia ha avanzado mucho en la identificación y atención de estas dificultades en estudiantes de pregrado de medicina, pero en estudiantes de posgrado no se había logrado una adecuada detección y acompañamiento. Esto debido a la gran carga académica y asistencial, incompatibilidad de horario, estigma, entre otros. Metodología Con el fin de favorecer un acompañamiento pertinente que propenda por el logro del proyecto académico y la formación integral de los estudiantes de posgrado, se les aplicó una encuesta, con el objetivo de encontrar mecanismos que permitan ampliar la oferta de Bienestar y realizar mejores intervenciones de ser necesario. Durante la semana de inducción fueron encuestados 122 estudiantes de posgrado de los 129 admitidos a las diferentes especializaciones médicas y quirúrgicas que se ofrecen en la Facultad de Medicina. Resultados Se determinaron aspectos socioculturales, académicos, sociales, de salud, del entorno social, emocionales y habilidades. Se fortaleció la oficina de bienestar con especialistas en psiquiatría, psicología, pedagogía, toxicología, sexualidad, terapia de pareja y de familia. Se programaron reuniones psicoeducativas con jefes de residentes para lograr un acercamiento por pares. Conclusiones Con estos resultados buscamos: $\bullet$ Realizar seguimiento desde los programas académicos a los estudiantes con dificultades sentidas específicas, con el fin de ayudarlos a mejorar su problemática y lograr un mejor desempeño en su vida personal, académica y profesional. Continuar con el seguimiento y orientación ofrecidos desde el Observatorio Estudiantil. • Evaluar el proceso que han tenido los estudiantes con dichas problemáticas y que han buscado acompañamiento desde Bienestar, con el objetivo de ver que tan efectiva ha sido la ayuda brindada por parte del grupo interdisciplinario. • Extender la invitación a los estudiantes con problemáticas identificadas para que hagan uso de los diferentes servicios que se ofrece desde la Oficina de Bienestar y Cultura. • Mantener comunicación continua con la Jefatura de Posgrados para hacer seguimiento y retroalimentar los procesos. 


\section{La experiencia del Hospital Universitario de Brasilia (HUB) y el tercer año de Residencia Médica en Pediatría.}

Picanço Marilucia

\section{(1) Universidade de Brasilia, Brasil.}

Introducción: El Programa de Residencia Médica (PRM) en el área de Medicina del Niño y del Adolescente (MCA) del Hospital Universitario de Brasilia (HUB) Universidad de Brasilia (UnB), tuvo inicio en 2014 y tiene como objetivo el progresivo perfeccionamiento profesional y científico, de habilidades y actitudes del médico en las varias áreas del conocimiento. La capacitación posibilita el ejercicio del desempeño ético y celoso de la profesión. El Proyecto es de acuerdo con las normas de la Sociedad Brasileña de Pediatría (SBP) en consonancia con el Consorcio Global de Educación Pediátrica - GPEC (Global Pediatric Education Consortium). Cuenta con la infraestructura del HUB y varios escenarios en la red pública de salud del Distrito Federal. La propuesta de la ampliación del tercer año en el currículo de la Pediatría pretende formar un pediatra generalista, humanista, crítico y reflexivo. El proceso incluye las perspectivas de formación en servicio y en red, evaluación continua y desarrollo profesional. Guiados por la integración global curricular, si mantener acuerdos con los países de América del Sur y Paraguay, así como los países de Europa, Portugal y Bélgica. Esta posibilidad permite además del intercambio de informaciones y conocimientos, una inmersión en otras culturas lo que posibilita al residente mayor perfeccionamiento de su actuación junto a la comunidad ya los equipos transdisciplinares. Objetivo: Presentar el nuevo modelo de residencia de tres años en Pediatría adoptado en el HUB / UnB / DF. Objetivos del Proyecto: Posibilitar al residente, prestar asistencia integral al niño desde el nacimiento hasta el final de la adolescencia, apoyado en conocimientos cognitivos sólidos y en habilidades psicomotoras y afectivas. Metodología: se inició en 2014 con el programa de tres años designadas solamente 09 instituciones en Brasil que tendrían la incumbencia de abrir un edicto para Residencia Médica en Pediatría de tres años. El desafío sería grande en función de que los otros programas, en el país, estén con programas de dos años. La evaluación se haría no sólo por las pruebas teóricas, de habilidades y actitudes en cada servicio, pero con una evaluación seriada de prueba teórica para titulación de especialista en tres años, (TEP - seriado) realizada y obtenida por la Sociedad Brasileña de Pediatría. Resultados: Hemos tenido en el período de 2014 a 2018 un total de 35 médicos residentes siendo en 2014 (06); 2015 (06) tenía 06 vacantes y llena todo en 2016 (07) 2017 (08) y 2018 tenía 08 vacantes y llenar todas las vacantes. De ese total tuvimos una desistencia en 2018 y una reprobación en el
TEP - seriado. Conclusiones: Los resultados demuestran la importancia del proyecto de tres años en la formación del pediatra y que el mismo está bien sedimentado y consolidado en las bases teóricas y prácticas de acuerdo con el currículo global, con las directrices RM de la Sla SBP, así como las directrices de la enseñanza médica.

\section{Diseño de un curso de fresado de hueso temporal para el aprendizaje de habilidades quirúrgicas en cirugía de oído para residentes de otorrinolaringología}

Thöne Natalie (1), Correa Diego (1), Orellana María (1), Huidobro Bárbara (1), San Martín José (1)

\section{(1)Pontificia Universidad Católica de Chile, Santiago, Chile}

Introducción: La educación médica del siglo XXI ha cambiado a un currículo basado en competencias, ejemplo es el modelo CanMEDS. Dentro de este el rol Médico Experto incluye el desarrollo de competencias o habilidades quirúrgicas en especialidades como Otorrinolaringología. La enseñanza y aprendizaje de estas habilidades es un desafío, porque depende de las oportunidades quirúrgicas y la necesidad de no comprometer la seguridad del paciente. En este contexto, la simulación surge como una alternativa segura, que permite estandarizar el aprendizaje y es una metodología de aprendizaje activo centrada en el estudiante. El objetivo de este trabajo es diseñar un curso de simulación para el aprendizaje de habilidades quirúrgicas en cirugías de oído para residentes de otorrinolaringología. Método: se utilizó el modelo de 6 pasos diseñado por David Kern y colaboradores. Resultados: 1. Identificación del problema y evaluación general de necesidades: la patología de oído es común en países en vías de desarrollo, y requiere muchas veces de resolución quirúrgica. Además, representa un porcentaje importante de la lista espera quirúrgica de la especialidad. 2. Evaluación de necesidades específicas: el registro quirúrgico de los residentes evidencia una gran dispersión respecto a la exposición quirúrgica en cirugías de oído dentro de una misma cohorte. Residentes y egresados del programa opinan que el desarrollo habilidades quirúrgicas en oído son necesarias y que falta mayor exposición y práctica en este tipo de cirugía y la simulación ayudaría a mejorar sus habilidades. 3. Metas y objetivos: este programa tributa a las competencias CanMEDS en el Rol Experto en Otorrinolaringología. Se definieron 4 objetivos de aprendizaje tanto cognitivos como psicomotores. 4. Estrategias Educacionales: los contenidos determinados fueron la anatomía hueso temporal y oído y técnicas quirúrgicas como mastoidectomía, entre otras. Para objetivos cognitivos se utilizará un manual y videos y para los psicomotores la simulación en modelos 
cadáveres 5. Implementación: se definieron recursos humanos y materiales para el desarrollo del curso. 6. Evaluación y feedback: se usó una pauta de observación tipo checklist para la evaluación del aprendizaje. Método Kirkpatrick para la evaluación del programa en 3 niveles: 1) Encuesta de satisfacción 2) Seguimiento del residente durante cada sesión aplicando instrumento de evaluación de aprendizaje 3) Transferencia de lo aprendido en pacientes reales con mismo instrumento. Discusión: Las habilidades quirúrgicas en patología de oído son una necesidad de aprendizaje y se debe garantizar su adquisición en todos los residentes de nuestro programa. Para esto se diseñó este nuevo curso en consonancia con el modelo CanMEDS y el perfil de egreso de especialistas en otorrinolaringología que nuestro país necesita. La simulación en cadáveres es una gran oportunidad para estandarizar el aprendizaje quirúrgico en los residentes y cumplir con los objetivos de aprendizaje del programa. También este nuevo curso contribuyó al diseño de nuevos instrumentos de evaluación para el aprendizaje y cuenta con un plan de evaluación para una mejora continua. Esperamos poder aplicar este diseño de curso para otras áreas de la especialidad y así mejorar el proceso de enseñanza y aprendizaje de nuestros residentes.

\section{Telepsiquiatría: una experiencia exitosa en Antioquia.}

Agudelo Angela (1), Garcia Juan (1)

(1) Universidad de Antioquia, Medellín, Colombia.

Introducción: Describir la experiencia de Telepsiquiatría en la facultad de medicina de la Universidad de Antioquia en los primeros 12 meses de su implementación en ocho municipios del departamento. Métodos: Estudio descriptivo, que incluyó 111 pacientes atendidos durante el primer año. Se diseñó un instrumento para evaluar la satisfacción de los pacientes y se empleó el instrumento de aceptación de tecnología para evaluar la de los profesionales de la salud. Resultados: Se realizaron 340 atenciones durante ese periodo a 111 pacientes, $70(63,1 \%)$ de ellos recibieron al menos 2 atenciones por telepsiquiatría en el primer año.

Resultados: Se evaluó la experiencia en una muestra representativa de 38 pacientes (34\%), quienes manifestaron una resolución del problema en $94,7 \%$ y una satisfacción alta (100\%). En el programa participaron 9 profesionales de la salud, los cuales estuvieron de acuerdo en que la tecnología es útil, fácil de usar y tienen la intención de seguir usándola.
Conclusión: Los sistemas de salud a nivel mundial no han dado una respuesta adecuada a la carga de trastornos mentales; por esto, estrategias como la telepsiquiatría se considera una modalidad de atención ideal para personas que viven en lugares remotos y tienen dificultad de acceso a los servicios de salud especializados, con adecuada aceptación.

\section{Diseño Instruccional para Curso Mixto en Patología Dual, Estudio de Caso}

Agudelo Angela (1), Acevedo Luis (1)

\section{(1) Universidad de Antioquia, Medellín, Colombia.}

Introducción: El aprendizaje virtual se refiere a cualquier modelo educativo que hace uso y aprovechamiento de las Tecnologías de la información y comunicación (TIC) que permite el aprendizaje a distancia. El aprendizaje mixto combina el aprendizaje virtual y presencial. Siguiendo las políticas del Ministerio de Educación nacional y de la Universidad en cuanto a la integración de las TIC para la educación y partiendo de una necesidad académica particular confirmada por encuestas realizadas a profesores y residentes, se decide crear un curso mixto sobre patología dual para los residentes de psiquiatría de la Universidad de Antioquia.

Métodos: Para su desarrollo se utilizó el modelo ADDIE, uno de los más utilizados para el diseño instruccional virtual o mixto y consta de 5 fases análisis, diseño, desarrollo, implementación y evaluación. Para el diseño instruccional del curso, se utilizó el Formato para la planeación de Ambiente Virtual de Aprendizaje (AVA Académico) de la maestría en telesalud de la Universidad de Antioquia, donde se especificaron los objetivos, metodología, material de estudio y actividades evaluativas de cada módulo. El curso está basado en el diseño de diferentes actividades que desarrollarán los estudiantes buscando la apropiación, análisis crítico y generación de conocimiento. Para su implantación se escogió la plataforma Moodle de código abierto, por su flexibilidad, escalabilidad, entorno intuitivo, arquitectura y porque es la que se utiliza en la Facultad de Medicina.

Resultados: La evaluación del diseño instruccional mostró facilidad de navegación, alta usabilidad y buena calidad técnica, estética y pedagógica.

Conclusiones: Para el adecuado desarrollo de un curso mixto o 100\% virtual es fundamental seguir un modelo de diseño instruccional. Se sugiere un próximo estudio para la evaluación de la satisfacción del usuario final y el cumplimiento de los objetivos de enseñanza propuestos al finalizar el curso. 


\section{Residentes de Medicina Familiar con dificultades de adaptación a la exigencia académica: una oportunidad para el desarrollo.}

Bizama Andres (1), Perez-Wilson Patricia (1)

\section{(1) Universidad de Concepción, Concepción, Chile}

Introducción: La Unidad de Apoyo al Residente (UAR) en la línea de apoyo a becados con dificultades asociadas a la exigencia académica y las crisis normativas y no normativas, realiza una intervención de fortalecimiento de recursos personales, considerando factores afectivo-motivacionales y habilidades cognitivas para el mejoramiento de condiciones de aprendizaje que potencien la calidad de razonamiento y que permitan un mejor afrontamiento a exigencias de la beca y a resolución de conflictos en la vida de los becados.

Métodos: Esta intervención se basa en la Experiencia de Aprendizaje Mediado (EAM) a través del Programa de Enriquecimiento Instrumental, dispositivo que propicia el fortalecimiento de recursos motivacionales, cognitivos y emocionales. El comité académico de la Beca ha derivado 4 médicos residentes para participar en este proceso, dadas sus dificultades de adaptación a la exigencia académica. Este entrenamiento se enmarca en el proceso del portafolio recuperativo, luego del cual terminada la intervención vuelven a ser evaluados para valorar la factibilidad de continuar la beca. La modalidad de trabajo es individual, con sesiones bisemanal por un período de 4 meses.

Resultados: La intervención de EAM mediante el Programa de Enriquecimiento Instrumental ha permitido desarrollar una intervención integral y sistemática para los residentes que han tenido dificultades académicas, contribuyendo a generar condiciones de mayor adaptación para su proceso de formación, así como para su vida en general. Los principales resultados reportados por los residentes son: a) A nivel motivacional: incremento de motivación intrínseca, autoeficacia, sentimiento de competencia, percepción de capacidad de cambio y menor temor al fracaso, b) A nivel cognitivo: incremento en habilidad para considerar 2 o más fuentes de información simultáneas, conducta comparativa espontánea, campo mental, búsqueda de evidencias lógicas, interiorización del comportamiento, planificación de la conducta y proyección de relaciones virtuales. Lo anterior les permite reinsertarse de manera satisfactoria a la beca, dando continuidad a su formación y manteniendo los logros alcanzados.

Conclusiones: La aplicación de la Experiencia de Aprendizaje Mediado a través del Programa de Enriquecimiento Instrumental, ha demostrado ser eficaz para fortalecer el desempeño integral de becados de medicina Familiar con dificultades académicas, logrando reintegrarse al programa formativo satisfactoriamente según reporte propio, de tutores y equipo docente. Asimismo, se observa desarrollo de conductas más adaptativas, con impacto para la especialidad. Creemos que este tipo de intervenciones está alineado con el sentido de la medicina familiar, acogiendo a las personas en dificultad con un abordaje integral, basado en sus propios recursos, modelando así relaciones de ayuda y desarrollo orientadas a fortalecer la autonomía las personas. Finalmente creemos que esta experiencia también contribuye a mejorar la gestión en el ámbito de la eficiencia de los recursos disponibles.

\section{Implementación del ejercicio como estrategia para la disminución del Síndrome de Burnout en residentes de pediatría.}

Chamorro Zaida (1), Martínez Gloria, Mesquita Mirta, Godoy Laura

(1) Hospital General Pediátrico Niños de Acosta Nu

Introducción: El síndrome de burnout es una reacción afectiva en respuesta al estrés laboral crónico que puede ocasionar deterioro o depleción de los recursos emocionales y cognitivos, culminando en un estado de agotamiento en el que el trabajador duda completamente de su capacidad para realizar sus labores, mostrándose cínico sobre el valor y el sentido de éstas. Objetivos: Evaluar el ejercicio como estrategia de intervención para reducir el grado de Síndrome de Burnout. Material y métodos: Estudio de intervención (actividad física), aleatorizado, abierto, realizado con residentes de pediatría, de noviembre 2016 a febrero del 2017. Se asignaron dos grupos: Grupo 1 que realizó actividad física (clases de zumba), durante 1 hora, 2 veces por semana (por 10 semanas) y Grupo 2: grupo control, que no realizó actividad física. Fueron excluidos aquellos residentes que no asistieron a las clases de zumba más de dos veces. Se entregó el cuestionario de Maslach a todos los residentes de pediatría antes y después de las clases de zumba, previo consentimiento informado. El tamaño muestral fue 21 participantes en cada grupo, para un error alfa de 0,05 y una potencia de $80 \%$. Los datos fueron analizados con SPSSv21, para la asociación de variables cualitativas se utilizaron: tabla de contingencia, prueba de Chi cuadrado de Pearson y test exacto de Fisher. La asociación de variables cuantitativas se realizó con el T de Student. Variables estudiadas: edad, sexo, año de residencia, convivencia, hijos, actividades recreativas, agotamiento emocional $(A E)$, despersonalización (DP), realización personal (RP), SB pre-intervención, SB post intervención Resultados: Fueron 
incluidos 47 residentes (16R1, 16R2, 15R3). La media de edad fue $27 \pm 2$ años. Predominio del sexo femenino $(85,1 \%)$. No hubo diferencias significativas en ambos grupos respecto a la convivencia, el grupo 1, vivía en su mayoría con su familia $(56,5 \%)$ vs sólo en el grupo 2 (37,5\%). En el Grupo 1: 95,7\% no tenía hijos vs $29,2 \%$ del Grupo 2 que si los tenía. Ambos grupos realizaban actividades físicas en bajo porcentaje (39,1\% y 29,2\%), sin embargo, la mayoría tenían actividades recreativas $82,6 \%$ y $75 \%$ respectivamente. En cuanto a los componentes del SB $(A E, D P, R P)$, pre intervención, no se encontraron diferencias significativas en ambos grupos (AE alto: $73,9 \%$ vs 70,8\%; DP alta: $73,9 \%$ vs $75 \%$; RP baja: $65,2 \%$ vs $58,3 \%$ ), en tanto que posterior a la intervención, se notó un descenso en el grupo 1 del AE de $73,9 \%$ a $47,8 \%(p=0,18)$ y de la RP de $65,2 \%$ a $30,4 \%$ $(p=0,06)$. Previa a la intervención el Grupo 1 tenía SB en $52,2 \%$ vs 37,5\% del Grupo 2, sin embargo, posterior a la intervención, se encontró SB en el Grupo 1 en 17,4\% ( $p=0,03$ Test exacto de Fisher). Conclusión: La intervención con el programa de ejercicio realizado en promedio una a dos veces por semana disminuyó el grado de Burnout de manera significativa en un periodo de diez semanas.

\section{Percepción de profesores y residentes de un hospital universitario de alta complejidad en Colombia, frente a la comunicación de malas noticias}

Torregrosa Lilian (1), GempelerFritz

\section{(1) Universidad Javeriana - Hospital San Igancio, Bogotá Colombia}

Introducción: El arte de comunicar malas noticias no es opcional hoy en día en medicina y constituye una competencia fundamental del profesionalismo médico. Pero tanto estudiantes como médicos reconocen deficiencias en la enseñanza curricular recibida sobre el tema y manifiestan tener poca preparación, sentir inseguridad, temor e incluso angustia moral al momento de enfrentar estas situaciones. Dado que no existe una última palabra sobre el método de enseñanza ideal, conocer las percepciones actuales de las generaciones de estudiantes y profesores sobre este tema, puede contribuir a la construcción colaborativa de una respuesta. Objetivo: Explorar las percepciones del cuerpo médico de un hospital universitario de alta complejidad, frente a la comunicación de malas noticias.

Metodología: Se planteó un diseño cuasiexperimental entre enero y diciembre de 2017 en el que participaron voluntariamente los médicos de planta y residentes programados por la institución para asistir a un taller de enseñanza multimodal sobre el tema, diseñado por el Servicio de Ética Clínica hospitalario y titulado
“Conversaciones difíciles en medicina: el arte de comunicar malas noticias". La participación consistió en el diligenciamiento de una encuesta anónima. El instrumento fue validado previamente e incluía los datos demográficos, así como preguntas de selección múltiple única respuesta que exploraban la percepción de los participantes sobre el tema. Resultados: La población estuvo conformada por 204 profesores y 219 residentes que diligenciaron la encuesta en forma completa, lo cual corresponde al $98 \%$ de los participantes en las sesiones educativas. La mayoría de los profesores y residentes consideró tener habilidad para comunicar malas noticias, si bien los primeros valoraron su capacidad por encima de los residentes. (78\% vs $52 \%$ ) En cuanto la forma en que adquirieron las destrezas para comunicar malas noticias se obtuvo lo siguiente: Residentes Profesores Entrenamiento formal 6,4\% 2,9\% Observación de sus docentes o colegas $65,8 \% 60,3 \%$ Ensayo y error $21,9 \% 23,5 \%$ Con respecto a las tareas más difíciles al momento de enfrentar malas noticias con los pacientes, los residentes y profesores coincidieron en que la principal era "Discutir problemas relacionados con el final de la vida (incluyendo ordenes de no resucitación y voluntades anticipadas)", Residentes 35,1\% y Profesores 30,4\%, seguida por "Hablar sobre la terminación de un tratamiento activo y el comienzo de un tratamiento paliativo" Residentes $27,4 \%$ y Profesores $24,5 \%$. Coincidieron ambos grupos en que el aspecto más difícil al comunicar malas noticias es lidiar con las emociones de cada paciente (llanto, rabia y ansiedad), así como que que los factores limitantes para una comunicación efectiva y adecuada de malas noticias son: el temor a las quejas y demandas legales y a la reacción emocional del paciente, así como la inseguridad de cada médico; la totalidad consideró a la educación formal sobre el tema una herramienta de ayuda en su práctica profesional. Conclusión: El diseño de estrategias curriculares efectivas para la adquisición de competencias en la comunicación de malas noticias puede enriquecerse al incorporar estrategias que respondan a las necesidades planteadas por los médicos en ejercicio.

Caracterización de los estilos de aprendizaje para los residentes asistentes a curso de formación para microcirugía en la Facultad de Medicina de la Universidad de Antioquia

Rojas Manuel (1)

(1) Universidad de Antioquia, Medellín, Colombia.

Introducción: la psicología educativa se constituye en la disciplina científica que ha dado soporte epistemológico a las diferentes tendencias en pedagogía y educación médica, a tal punto que muchos 
de los enfoques pedagógicos, los procesos que intervienen en la formación cognitiva, procesos de aprendizaje concentran el mayor interés en las distintas perspectivas planteadas por la psicología educativa, desde esta dinámica surge el interés de implementar estos avances en los modelos formativos para microcirugía y con ello determinar los estilos de aprendizaje de los estudiantes asistentes como elemento transversal para las metodologías propuestas, como punto de partida que busca responder el objetivo principal, cuál es el estilo de aprendizaje de los residentes de diferentes especialidades médico quirúrgicas que hacen parte del modelo formativo en microcirugía para el laboratorio.

Métodos: como marco en la investigación descriptiva en la cual participaron 26 residentes de especialidades médico - quirúrgicas en cirugía plástica, ortopedia y traumatología, neurocirugía, trasplantes, cirugía general y maestría en ciencias veterinarias, quienes hacen parte del programa de formación en técnicas en microcirugía del laboratorio de la facultad de medicina de la Universidad de Antioquia, a cada estudiante se le ofrece un cuestionario CAMEA-40 el cual contiene con información de la trayectoria académica, una serie de 40 preguntas con ítems de respuesta siempre, casi siempre, muchas veces, algunas veces y nunca, con estas preguntas busca identificar a cada estudiante en los cuatro estilos reflexivo, teórico, pragmático y activo; como principio y único fin en implementar estrategias metodológicas que fortalecieran el proceso cognitivo para cada actor, lo que permite elaborar perfiles y diseñar estrategias que posibiliten mejorar el rendimiento, dirigiendo las acciones de la práctica docente pueden estar dirigidas eficazmente en el proceso enseñanza aprendizaje.

Resultados: mediante la implementación de los residentes asistentes al cuestionario CAMEA-40 nos permitió identificar el principal estilo de aprendizaje es de tipo teórico con el $62 \%$, reflexivo con un $27 \%, 11 \%$ pragmático y $0 \%$ activo; como segunda tendencia de estilo predominó reflexivo $35 \%$, teórico un $34 \%$, pragmático $23 \%$ y activo $8 \%$.

Conclusiones: los Estilos de enseñanza de los docentes tienen incidencia en la forma en que aprenden los estudiantes, una vez que han sido identificados de manera cualitativa se dirigen acciones de práctica dirigidas precisamente a la fase del proceso de aprender, incorporando ante cada estudiante un método idóneo en el estilo particular como bastión del docente, complementando un espacio de conocimiento de sus aprendices, obedeciendo al uso de dinámicas pedagógicas como baluarte en los procesos de enseñanza aprendizaje. Por ello es de vital importancia implementar ante los objetivos propuestos al curso, estrategias de enseñanza con base en los estilos singulares como herramienta esencial en el diseño de metodologías y didácticas que facilitan la labor docente en pro del desarrollo de cursos de alta calidad para los residentes de nuestras especialidades en técnicas en microcirugía.

\section{Prevalencia y detección de violencia de pareja por residentes de Medicina Interna en Chile}

Gómez Saúl (1), Capurro Daniel (1), Torres Rafael (1), Ruiz Sergio (1)

(1) Pontificia Universidad Católica de Chile, Santiago, Chile.

Introducción: La violencia de pareja (VP) es un fenómeno frecuente que tiene graves efectos en la salud mental y física. Teniendo en cuenta su importancia, es relevante identificar su prevalencia y la capacidad de los médicos generales en formación para detectarlo. Objetivos: Explorar la prevalencia y detección de VP en un servicio de atención ambulatoria atendido por residentes de Medicina Interna (MI); Explorar su asociación con sintomatología depresiva; Comparar y discutir programas de formación de MI respecto de la detección de VP.

Métodos: Se realizó un análisis transversal de primeras consultas en MI ( $n=203$ ). Se usaron escalas de violencia (ISA) y depresión (PHQ9). La detección fue evaluada posteriormente mediante revisión del registro clínico. Además, se realizó una revisión cualitativa de distintos programas de formación en MI.

Resultados: La frecuencia total de VP fue 20\% (16.8\% VP no física y $10 \%$ VP física). No se encontraron diferencias estadísticamente significativas entre sexos para la VP tanto física $(X 2(1)=0.35, p=$ .56) como no física (X2 (1) = 0.71, $p=.4)$. La frecuencia detectada de depresión fue 22,2\%. La gravedad de la VP física y no física se asoció con la gravedad del trastorno depresivo $(r=.209, p=.004 y$ $r=.247, p=.001$, respectivamente). No se identificaron elementos escritos sugerentes de detección de VP por parte de los residentes. En el análisis de contenido de los programas de formación de MI de tres países (Chile, Canadá y España) no se encontraron conceptos relacionados con VP.

Conclusiones: La VP es un factor de alta prevalencia, con muy baja detección por residentes. La presencia de VP podría afectar a ambos sexos con similar incidencia y asociarse a síntomas depresivos. Los programas de formación en MI analizados carecen de contenidos relacionados con violencia por lo que es imperativo incluir objetivos de aprendizaje orientados a la detección de este fenómeno. 


\section{Los residentes consultan frecuentemente información científica, pero no la entienden. Un desafío para el logro del rol Académico (CanMEDS).}

Neumann Ignacio (1), Rivera Solange (1), Rojas Luis (1), Karzulovic Lorena (1)

(1) Pontificia Universidad Catolica de Chile, Santiago, Chile.

Introducción: De acuerdo al modelo de competencias CanMEDS, una práctica clínica de excelencia involucra dos competencias claves: el compromiso con el aprendizaje continuo y la capacidad de integrar a la práctica la mejor evidencia disponible. Estas competencias requieren que el médico sea capaz de evaluar críticamente la integridad, la confiabilidad y la aplicabilidad de la investigación relacionada con la salud. Habitualmente, la evidencia científica generada se presenta utilizando conceptos estadísticos y epidemiológicos, que no resultan intuitivos para los clínicos. Esto ha motivado a la incorporación de cursos de análisis crítico y medicina basada en evidencias a los programas de formación de residentes. A pesar de lo anterior, todavía parece existir una brecha en la capacidad de los residentes para interpretar correctamente los resultados de la investigación científica. Objetivo: Evaluar la capacidad de entender la evidencia presentada en escenarios clínicos en un grupo de residentes de medicina interna y familiar.

Métodos: Realizamos una encuesta a residentes de medicina interna y medicina familiar de 9 países (Argentina, Arabia Saudita, Canadá, Chile, Estados Unidos, España, El Líbano, Noruega y Suiza). En la encuesta recabamos información demográfica relevante, así como la frecuencia de uso de evidencia. Además, se les presentó 2 escenarios clínicos breves acompañados de una tabla de resumen de evidencia confeccionada según las recomendaciones del GRADE Working Group y la Colaboración Cochrane. Mediante preguntas de opción múltiple se evaluó el grado de comprensión de conceptos básicos de la evidencia presentada: efectos de la intervención, intervalo de confianza y certeza de la evidencia

Resultados: Encuestamos a 265 residentes, con una edad promedio de 28.3 años (desviación estándar 2.6 años). Aproximadamente la mitad fueron mujeres. El 49.8\% no había realizado ningún curso de análisis crítico o medicina basada en evidencias durante su formación. Un 48.6\% había realizado al menos un curso breve, y un 1.5\% tenía un entrenamiento formal en investigación (magíster o doctorado). La mayoría de los encuestados declararon consultar frecuentemente evidencia científica: $59.6 \%$ al menos una vez a la semana y un $24.2 \%$ al menos una vez al mes. Sin embargo, pese al uso frecuente, solamente un $41.1 \%$ fue capaz de entender correctamente la información presentada en las tablas de evidencia. El intervalo de confianza y la certeza de la evidencia resultaron ser los conceptos de mayor dificultad. No observamos variaciones significativas en nuestros resultados en relación con el país de origen ni con haber realizado un curso de análisis crítico o medicina basada en evidencias durante su formación

Conclusión: Nuestro estudio muestra que la mayoría de los residentes no es capaz de entender correctamente información científica, lo que es fundamental para la toma de decisiones clínicas de acuerdo a la mejor evidencia disponible. Creemos que los programas de formación debieran poner mayor énfasis en entregar herramientas que permitan a los médicos comprender e incorporar la evidencia en la toma de decisiones.

\section{Residencia Uni y Multiprofisional de Salud de la Cuidad de São Paulo- Brasil: Puntos Fuertes y Dificultades}

Nogueira Valnice (1)

(1) Secretaria Municipal da Saúde de São Paulo, Brasil.

Introducción: Los programas de Residencia Uni y Multiprofesional en Salud en Brasil tienen como premisa calificar profesionales del Sistema Único de Salud y promover la integración enseñanza-servicio en los niveles medio y superior y, beneficiar al usuario, a la familia y la comunidade. El municipio de São Paulo con aproximadamente 12 millones de habitantes forma parte del Grupo 2 del Índice Paulista de Responsabilidad Social y posee particularidades que hacen la construcción del conocimiento y las experiencias de los residentes de forma impar. Objetivos: Describir acerca de los programas de Residencia en Salud de la cuidad de São Paulo e evaluar los puntos fuertes y dificultades. Metodo: Estudio descriptivo reflexivo realizado en el primer trimestre de 2018. Creado un formulario con 13 ítems para la compilación y posterior análisis de los datos. Resultados: Los Programas de Residencias uni y multiprofesional fueron creadas en 1983 y 2015 y tienen una duración de 3 y 2 años respectivamente. Los escenarios de práctica son en el área hospitalaria y atención básica y los programas ofrecidos son Bucomaxilofacial, Urgencia / Emergencia, Neonatología, Terapia Intensiva y Prácticas Integrativas y Complementarias en Salud. Existen 11 categorías profesionales de las 13 autorizadas por los Órganos competentes: Biología, Educación Física, Enfermería, Farmacia, Fisioterapia, Fonoaudiología, Nutrición, Odontología, Psicología, Servicio Social y Terapia Ocupacional. Se ofrecen anualmente 68 vacantes por medio de proceso selectivo y las profesiones que poseen el mayor número de inscritos son los Enfermeros, Odontólogos y Psicólogos. Las que menos se someten a la selección son los Educadores Físicos y 
Fonoaudiólogos. La tasa de evasión por año de residencia está en torno al 25\%, siendo El Programa de Terapia Intensiva es el que más posee desistencia y los Enfermeros y Psicólogos las categorías que más formalizan el cierre. El programa de Cirugía Bucomaxilo facial es el que menos tiene salida de residentes. Entre las fragilidades destacan las cuestiones relativas a la estructura de los servicios (recursos humanos, materiales y físicos), al proceso (calificación y valorización de preceptoria, necesidad de reorganización del proceso de trabajo, realización de educación permanente). Se enumeran los puntos fuertes: Espacio que posibilita la producción de conocimiento, saberes y el uso de investigación en beneficio de la práctica; b) Fortalecimiento del proceso de educación permanente en salud, de las relaciones interpersonales y mejor cualificación de los profesionales y de los servicios. Conclusión: Los programas de residencia multiprofesional han proporcionado transformaciones significativas en el proceso de trabajo de los profesionales y de la comunidad en general. Además, suscita la reflexión de los diversos niveles de gestión en beneficio de la reorganización de los programas y servicios.

Diseño de un Curso Transversal de Formación en Docencia Clínica en Modalidad Semi-Presencial Para Residentes de Especialidades Médicas de la Pontificia Universidad Católica de Chile

Pérez Francisco (1), Leiva Isabel (1)

(1) Pontificia Universidad Católica de Chile, Santiago, Chile.

Introducción: La docencia clínica (DC) es esencial para la enseñanza-aprendizaje en medicina. Los residentes cumplen con un rol fundamental dentro de la docencia a estudiantes. Este rol docente es reconocido en la literatura internacional, lo cual ha llevado a la creación de cursos de formación en habilidades docentes para residentes, con muchas experiencias publicadas a nivel mundial. En nuestro país, sin embargo, no se ha visto esta tendencia, existiendo sólo una experiencia publicada. Objetivo: Diseñar un curso transversal de formación en DC, en modalidad semi-presencial, para residentes de especialidad y subespecialidad médica de la UC.

Métodos: Este proyecto educacional se dividió en siete etapas, basadas en el modelo de desarrollo curricular de Patricia Thomas y David Kern. El diseño del curso se realizó mediante 'diseño en reversa', a partir de una revisión de la literatura y un análisis de necesidades institucional, que incluyó encuestas a residentes, jefes de programa y la Dirección de Postgrado. Resultados: Primero, se delimitó la brecha entre la 'situación ideal' y la 'situación real' de la docencia realizada por residentes en la UC, destacando la falta de un programa de formación. Luego, se realizó un mapeo de información a partir de los programas identificados, destacando 'Feedback' en cuanto a contenidos, 'Role-play y/o simulación' como metodología de enseñanza-aprendizaje y 'Examen de Docencia Objetivo Estructurado' ('OSTE') como método de evaluación. Las encuestas permitieron constatar la valoración y apoyo transversal que existe a la DC realizada por residentes. Posteriormente, se definieron cuatro resultados de aprendizaje, que a su vez permitieron definir los objetivos de aprendizaje para el programa. Se incluyeron contenidos como'Retroalimentación','Docencia bedside,'Modelaje'y'Habilidades Comunicacionales Docentes'; se incorporaron metodologías activas como la 'clase invertida'y se optó por métodos de evaluación en los niveles más altos de la pirámide de Miller, dentro de los que se incluyó un OSTE. Además, se decidió implementar el curso en modalidad semi-presencial. Finalmente, se desarrolló un plan de implementación, considerando los recursos existentes, el apoyo institucional, la estructura administrativa necesaria y las potenciales barreras; y se adaptó un modelo de Kirkpatrick para la evaluación del programa.

Resultados: El programa fue diseñado en base a una exhaustiva revisión de la literatura y a un análisis de necesidades institucional desde el punto de vista de los principales interesados, por lo que se prevee una implementación sea exitosa. Este curso debiera beneficiar a los residentes y sus estudiantes, con algunos beneficios además para los jefes de programa y la Dirección de Postgrado en términos de acreditación institucional. A futuro, se esperaría la creación de cursos específicos para cada especialidad, longitudinales y con refuerzos periódicos, así como la definición de funciones docentes específicas para los residentes. Existen algunas limitaciones importantes a considerar, destacando el limitado número de residentes que accede al programa, la necesidad de capacitar a los tutores y los recursos necesarios.

Conclusión: Este proyecto busca ser una primera aproximación al problema identificado. A futuro, se espera que nuevas iniciativas contribuyan a una solución definitiva.

\section{Promoción de la salud en Dermatología: un estudio de encuestas sobre la experiencia de residentes y docentes en un programa de formación chileno}

Saldías-Fuentes Cristóbal (1), Sabatini-Ugarte Natalia (1), AbarzúaAraya Álvaro (1), Vera-Kellet Cristian (1), Navajas-Galimany Lucas (1).

(1) Departamento de Dermatología Pontificia Universidad Católica de Chile, Santiago, Chile.

Introducción: En distintos trabajos se ha reportado que el rol "promotor de la salud" se encuentra entre los más difíciles de enseñar 
y evaluar, particularmente por falta de un marco curricular o parámetros para evaluar dichas competencias. La literatura disponible en la materia es escasa, y hasta la fecha no existen publicaciones de esta índole en Chile. El objetivo es describir la situación de enseñanza y evaluación del rol "promotor de la salud" en el servicio de Dermatología de la Pontificia Universidad Católica de Chile.

Métodos: Estudio transversal a través de encuestas a residentes y médicos staff del servicio de Dermatología de la Pontificia Universidad Católica de Chile. Encuesta de 2 partes: la primera para antecedentes demográficos; la segunda en forma de escala de Likert para objetivos específicos. Se obtuvo respuestas a través de plataforma web, anónima, confidencial, voluntaria, previo consentimiento de cada participante. Análisis estadístico con programa SPSS Statistics v21 a través del test estadístico Chi-cuadrado para variables categóricas y T-Student para variables continuas. Se consideró estadísticamente significativo $p \leq 0,05$.

Resultados: Se obtuvo respuesta de 38 médicos staff y 22 residentes. El 55,3\% de los médicos staff tenían cargo académico. 7 residentes cursaban primer año, 8 segundo año, y 7 tercer año. Del total, 53,3\% aseguraron tener algún nivel de formación en docencia (65,8\% médicos staff y $31,8 \%$ residentes). Entre los participantes, $94,7 \%$ de staff y $86,4 \%$ de residentes estuvieron de acuerdo o totalmente de acuerdo con que la promoción de salud y la prevención de enfermedades juegan un papel sustancial en la práctica de la Dermatología. Un 97,4\% del staff y 95,5\% de los residentes, afirmaron que estos temas son importantes de abordar durante la residencia, y que es necesaria una capacitación formal al respecto. El 36,8\% del staff y ninguno de los residentes declararon manejar información con respecto a grupos comunitarios o asociaciones destinadas a promoción de la salud en Dermatología ( $p$ 0,026). Un $81,6 \%$ del staff y un $90,9 \%$ de los residentes tenían conciencia de la existencia del rol promotor de la salud dentro del programa de formación basado en competencias CanMEDS. El 58,3\% de los encuestados consideró que hay una formación formal de dicho rol en el programa actual de Dermatología. El 90,9\% de los residentes afirmaron haber participado en actividades u organizaciones de promoción de salud y/o prevención de enfermedades antes de la residencia, mientras que durante la residencia la cifra fue de 40,9\%.

Conclusión: Existe casi unanimidad en reconocer la importancia del rol de promotor de la salud en la práctica clínica diaria y la necesidad de tener instancias de enseñanzas y evaluaciones formales. La baja participación de residentes estas actividades durante su residencia (respecto a la participación en el pasado) debe tenerse en consideración. Resultados similares son esperables en otros roles que no han sido incorporados históricamente en las mallas curriculares de medicina, por ejemplo, los roles colaborador, comunicador y líder.

\section{Importancia del Jefe de Residentes en un Departamento de Medicina Interna: notas de una experiencia en Uruguay}

Ferreira Joaquín (1)

(1) Medicina Interna, Hospital Maciel, Montevideo, Uruguay.

Introducción: La presencia de un Jefe de Residentes que cumpla con diversos aspectos organizativos dirigidos a mejorar el funcionamiento del grupo de residentes es fundamental. En diversos países la figura del Jefe de Residentes ocupa un rol preponderante en la estructura académica, ejerciendo un puesto de enlace entre los residentes, docentes y la estructura administrativa del hospital. El Hospital Maciel es un hospital público de tercer nivel localizado en la ciudad de Montevideo, Uruguay, asociado a la Facultad de Medicina de la Universidad de la República. En el Hospital Maciel funcionan dos servicios clínicos dependientes del Departamento de Medicina Interna, las Clínicas Médicas «1» y «3». Cada servicio cuenta con un cuerpo docente integrado por 14 facultativos. Un total de 20 residentes de Medicina Interna (10 en cada servicio) cursa cada año, correspondiente a residentes de primer, segundo y tercer año de la especialidad. Cada año se provee un cargo de Jefe de Residentes de Medicina Interna mediante concurso de oposición y méritos. Dicho cargo se encuentra vacante en los últimos dos años.

El objetivo de este trabajo es describir por primera vez las principales funciones desempeñadas por el Jefe de Residentes de Medicina Interna en el Hospital Maciel en el período comprendido entre noviembre de 2015 y noviembre de 2016, y enfatizar la importancia de contar con esta figura de referencia durante la etapa de formación de los médicos especialistas.

Métodos: Estudio cualitativo con diseño documental.

Resultados: Creación de una base de datos Se recolectaron datos demográficos de los residentes mediante una encuesta escrita y se conformó una base de datos que permitió tener su conocimiento integral.

Establecimiento de un canal de comunicación Se estableció el correo electrónico como principal medio de comunicación formal entre el Jefe de Residentes, docentes y residentes. En forma alternativa se utilizó un grupo de WhatsApp.

Creación de una Comisión de Residentes Se designó mediante votación un representante del primer, segundo y tercer año de la residencia, para conformar una Comisión de Residentes. Esta comisión se reunió en forma periódica con el Jefe de Residentes para discutir diversos temas de interés (p.ej. cumplimiento del 
programa de la residencia, plan de trabajo, propuestas de mejora, resolución de conflictos, etcétera).

Coordinación y desempeño de actividades asistenciales Se coordinaron las actividades de consulta ambulatoria y guardias de urgencia en conjunto con los jefes de servicio. Asimismo, el Jefe de Residentes se mantuvo ligado a la realización de tareas asistenciales en estos escenarios y en salas de internación.

Desarrollo de actividades formativas a) Seminarios. Se organizaron tres ciclos con un total de 23 seminarios preparatorios para las pruebas teóricas semestrales en las áreas de hematología, reumatología, cardiología y neumología, supervisados por docentes de los servicios de Hematología, Terapia transfusional, Cardiología, Neumología y Medicina Interna. b) Curso de razonamiento clínico. Se planificó un curso de razonamiento clínico orientado a potenciar las habilidades de los residentes en esta competencia. Se completaron nueve sesiones clínicas en total. El aprendizaje basado en casos fue la metodología de enseñanza utilizada, y se trabajó con pacientes reales. Esta actividad presentó niveles de satisfacción global superiores al 90\% entre residentes y docentes. c) Curso de introducción a la medicina basada en la evidencia. Este curso se implementó con el objetivo de educar a los residentes en herramientas para el análisis crítico de la información biomédica. Se realizaron cuatro exposiciones teóricas a cargo de docentes de Clínica Médica y del Departamento de Métodos Cuantitativos de Facultad de Medicina, así como de un experto nacional. d) Primera Jornada de desnutrición hospitalaria y prescripción nutricional. Esta actividad se organizó en conjunto con la Unidad de Nutrición Especial para concientizar al equipo asistencial acerca de la importancia de la detección precoz del paciente internado con riesgo nutricional.

\section{Redacción de la Guía de anticoagulación del Hospital Maciel}

El Jefe de Residentes colaboró directamente con los servicios de Medicina Interna y Hematología para la redacción final de esta guía, adaptando su versión extendida a un formato de bolsillo para su difusión.

Conclusión: La información expuesta refleja que el Jefe de Residentes desempeñó variadas funciones administrativas y asistenciales durante el período descrito. Además, el Jefe de Residentes contribuyó a la planificación, desarrollo y ejecución de diversas actividades académicas destinadas a los residentes, en el marco del Programa de Formación de Especialistas de Medicina Interna. El rol articulador y motivador del Jefe de Residentes en este tipo de actividades constituyó una pieza clave para el estímulo permanente hacia una mejor educación, brindando al mismo tiempo la contención necesaria en los aspectos humanos de los profesionales en formación. Es imprescindible dar trascendencia a esta figura de enorme valor, dado que su continuidad puede redundar en un impacto positivo para la calidad de la formación durante la residencia.

\section{Interpretación de la identidad profesional del educador médico como miembro de un proyecto educativo}

López Mildred (1), Olivares Silvia (1), Heredia Yolanda (2)

(1) Tecnológico de Monterrey, Escuela de Medicina y Ciencias de la Salud, (2) Tecnológico de Monterrey, Escuela de Humanidades y Educación

Introducción: Además de compartir saberes, valores y actitudes, los miembros de una profesión comparten la forma de entenderse a sí mismos, y la perspectiva de concebir la vida, esto se conoce también como identidad profesional. Para lograrlo, adquieren múltiples roles y responsabilidades, y se desempeñan colaborativamente, definiendo así su cultura profesional.

Esto es palpable con el educador médico quién además de estar comprometido con el desarrollo de sus estudiantes, es líder en su campo disciplinar y tiene actividades académicas o asistencias que demuestran su compromiso con la comunidad. Para Van den Berg y Lombarts (2018), actuar como un educador médico es diferente de serlo, y la diferencia radica en la internalización de los valores asociados a su rol, es decir la apropiación de su identidad profesional. El objetivo de este estudio fue explorar el concepto de identidad profesional a través de la cultura profesional que percibe el educador médico.

Métodos: Se utiliza un enfoque cualitativo, con una encuesta electrónica utilizando el cuestionario de Steinert (2012). La muestra consistió en 39 educadores médicos. Se realiza un análisis contenido de las respuestas, con el modelo de cultura profesional utilizando las categorías: individualismo, balcanizada, colegialidad por proyecto y colaboración entendida (Hargreaves y Fullan, 1991). La cultura de individualismo se refiere a un individuo altamente capaz que trabaja solo porque se siente independiente, en lugar de ser interdependiente como miembro de un todo más grande (Hosfstede, 2018); sus obligaciones o responsabilidades se deben hacia sus clientes directos. La cultura balcanizada describe subgrupos que comparten una formación especializada y tienen una interacción intermitente, sus obligaciones son también para sus jefes directos. La cultura de colegialidad por proyecto se refiere a individuos que colaboran alrededor de una meta específica, su designación como 
equipo fue realizada por un directivo en la organización. Por último, la cultura de colaboración extendida engloba los individuos que tienen un compromiso con la sustentabilidad de la organización. Representando una red estrechamente entramada en la que los individuos esperan que los miembros de su grupo demuestren lealtad hacia el colectivo.

Resultados: Las tendencias encontradas indican que la identidad de los profesores se asocia en: un 44\% al individualismo, 31\% balcanizada, $13 \%$ colegialidad por proyecto, y $13 \%$ colaboración extendida. Esta concentración en la cultura profesional hacia la individualidad y la cultura balcanizada se evidencia en el enfoque que como universidades se otorga a las responsabilidades individuales de los docentes, por ejemplo, al recibir retroalimentación y compensación en base a evidencias como: publicaciones, premios y resultados de las encuestas de alumnos.

Conclusión: En la medida que se haga la transición hacia actividades colaborativas con proyectos significativos, será posible obtener esta visión compartida de lo que significa ser un educador médico. La percepción de la identidad como un constructo sólido e inamovible ha evolucionado a una concepción dinámica donde el individuo posee múltiples facetas que conforman su identidad (Goldie, 2012). De ahí que se describe a la identidad como múltiple, dinámica, situacional y negociable. Los médicos que tienen una participación en la formación de recursos humanos en salud, deben balancear al menos dos facetas: la asistencial y la docente.

\section{Evaluación de la implementación de un} Journal Club en el Hospital Sótero del Río: facilitadores y obstáculos para la adquisición de los objetivos de aprendizaje.

Nitsche Pía (1)(2), Sommerhoff Constanza (1)(2), Alvo Daniela (3), Moreno Fernanda (4), Correa Matías (3)(4)

(1) Departamento de Psiquiatría (2)Pontificia Universidad Católica de Chile, (3)Complejo Asistencial Dr. Sótero del Río, (4)Universidad Diego Portales. Santiago, Chile.

Introducción: El Journal Club es una metodología de enseñanza-aprendizaje que se ha utilizado para diferentes necesidades en los programas de residencia. Su efectividad depende de diversas variables y de los objetivos de aprendizaje establecidos. El objetivo de este trabajo es evaluar los facilitadores y obstáculos para la adquisición de objetivos de aprendizaje en el Journal Club organizado en el Hospital Sótero del Río.
Métodos: Se aplicó una encuesta vía mail a los residentes y ex residentes de psiquiatría adulto e infantojuvenil que hayan participado en la actividad Journal Club entre abril del 2016 hasta julio del 2018.

Resultados: 38 residentes contestaron (95\%). 24 residentes tenían como lugar de formación la Pontificia Universidad Católica de Chile. La actividad fue valorada con un 5,5, en una escala entre el 1 a 7. Los principales facilitadores fueron la constancia del lugar y horario, el tiempo protegido para asistir y la discusión enriquecedora. Las principales barreras identificadas fueron la falta de competencias para el análisis crítico de la literatura y la metodología aplicada. Por último, de los objetivos de aprendizaje establecidos para la actividad, los residentes valoraron con mayor puntuación de logro de aprendizaje: “Exponer una publicación ante pares y docentes de manera efectiva y atractiva", e "Identificar publicaciones recientes que respondan a una pregunta de interés en la práctica clínica".

Conclusión: Los resientes valoraron esta metodología de enseñanza-aprendizaje y recomendaron potenciar la actividad a través de la elección del estudio acorde a un caso clínico, y una homogeneización en las habilidades de análisis crítico de la literatura y metodología de investigación.

\section{Evaluación de la calidad de las rotaciones clínicas percibida por los residentes de Medicina Interna: Desarrolloy propiedades psicométricas del IMPRINT-15}

Díaz Luis (1), Sepúlveda Paola (1), Abbott Eduardo (1), FuentesLópez Eduardo (1), Letelier Luz María (2), Riquelme Arnoldo (2) (3), Aizman Andrés (1)

(1) Departamento de Medicina Interna, (2) Departamento de Ciencias de la Salud, (3) Departamento de Gastroenterología. Pontificia Universidad Católica de Chile. Santiago, Chile.

Introducción: Las rotaciones clínicas son un componente esencial de los programas de especialización en Medicina Interna (MI), donde los objetivos curriculares se llevan a cabo a través de visitas docentes a pacientes hospitalizados y la supervisión de pacientes ambulatorios. Actualmente, no existen instrumentos validados para evaluar las rotaciones clínicas de MI. Por lo tanto, los objetivos de nuestro estudio fueron: 1. Desarrollar un instrumento para residentes con el fin de evaluar la calidad de las rotaciones clínicas en los programas de especialización en Ml; 2. Evaluar las propiedades psicométricas del instrumento. 
Métodos: Se utilizó metodología mixta, incluyendo una fase cualitativa y posteriormente una cuantitativa. Un grupo de expertos propuso ítems, basados en las necesidades previamente identificadas en las rotaciones clínicas de nuestro programa de MI. Posteriormente, se desarrolló una fase cuantitativa para generar consenso entre docentes y residentes, para definir que ítems debían ser incluidos en el instrumento (panel Delfi). Una vez generado el instrumento, se realizaron evaluaciones psicométricas para evaluar la validez de constructo (análisis factorial) y confiabilidad (coeficiente de confiabilidad de Raykov). Adicionalmente, comparamos los resultados obtenidos entre las diferentes rotaciones del programa de Ml.

Resultados:Tras dos rondas Delfi se obtuvo un instrumento de 15 ítems: Internal Medicine Program Instrument 15-items (IMPRINT-15). Sesenta y dos residentes contestaron 428 encuestas durante siete meses, utilizando una escala Likert (tasa de respuestas 98.9\%). La mediana fue 4,3 ([IQ] 3,9-4,7) (escala de 1 a 5). El análisis factorial evidenció dos dominios: 1. Docencia y actividades clínicas; 2 Evaluación y feedback. El instrumento es confiable con un coeficiente de Raykov de 0,86. Asimismo, el coeficiente de Raykov para los dominios fue de 0,89 y 0,83, respectivamente. La mejor rotación en nuestra residencia fue la "Unidad de Intermedio", mientras que hubo más aspectos a mejorar en "Sala de baja complejidad - Hospital privado".

Conclusión: El instrumento IMPRINT-15 es un cuestionario bi-dimensional, válido y confiable para evaluar la calidad percibida por los residentes de las rotaciones clínicas en Medicina Interna. Además, este constituye el primer instrumento validado con este fin a nivel mundial.

\section{Proceso de actualización de los programas de residencias médicas en la República de Panamá, con fines de acreditación 2017-2018}

\author{
Saied Maria (1), Noriega Lorena (2), Gutierrez Raquel (3)
}

(1)Curriculista de la Facultad de Medicina, Universidad de Panamá, (2) Docente Clínico del Complejo Hospitalario Dr Arnulfo Arias Madrid, Caja de Seguro Social, (3)Coordinadora de la Unidad de Postgrados clínicos de la Facultad de Medicina, Universidad de Panamá.

Introducción: En la República de Panamá existen actualmente 50 programas de residencias médicas que se desarrollan en hospitales del Ministerio de Salud y de la Caja de Seguro Social a nivel nacional. Estos programas de formación, fueron creados progresiva y de característica hospitalarios hasta 1984, cuando se obtiene aval universitario por la Facultad de Medicina de la Universidad de Panamá (UP), con poca ingerencia de la universidad en los procesos de formación; luego en 2003, se formalizan 6 programas como maestrías clínicas universitarias; Medicina Interna, Cirugía General, Ginecología y Obstetricia, Pediatría, Medicina Familiar y Psiquiatría, con mayor ingerencia de la universidad al incluir cursos universitarios obligatorios y sustentacion de trabajo final que comienzan oficialmente en 2011. En el año 2006, la Universidad de Panamá aprueba la equivalencia universitaria a los programas de especialidades no básicas y subespecialidades, hecho que abre la posibilidad de su apertura a nivel de maestrías y doctorados.

En 2014 se establece por resolución de Ministerio de Salud, la Comisión Nacional de Docencia, como un organismo de carácter científico y de coordinación de procesos académicos, responsables de planificar, organizar, integrar, controlar y dirigir los procesos de capacitación y acreditación de los médicos internos y residentes. En dicha comisión, tienen participación autoridades y directivos de hospitales de las instituciones formadoras, médicos residentes, colegio médico y la facultad de medicina de la UP.

La Facultad de Medicina, responsable de los procesos académcios y consciente de la necesidad de contar con programas nacionales únicos, inicia en octubre 2017, el proceso de actualización y formalización con fines de acreditación de los programas existentes y de nuevas aperturas de residencias médicas del país.

Métodos: El proceso de actualización se desarrolla en 6 fases que son:

Fase 1-Talleres de Sensibilización a los responsables de los programas. Fase 2-Evaluación y Autoevaluación de programas de residencias de las sedes hospitalarias habilitadas en el país.

Fase 3- Asesoría por especialidad para estructuración de la malla curricular del nuevo programa.

Fase 4- Presentación y aprobación por entidades competentes, Junta de Facultad de Medicina de la UP, Consejo Técnico de Salud del Ministerio de Salud.

Fase 5-Implementación de los programas.

Fase 6-Evaluación y Acreditación.

Resultados: Fase 1: Sensibilización: Se realizaron 4 talleres entre octubre y diciembre de 2017 con participación de Universidad de Panamá, Colegio Médico de Panamá, ministerio de salud, abordando temas como: Acreditación de programas qué y por qué, formación por competencia, regulaciones vigentes e incentivos. Participaron 150 médicos tutores.

Segunda: Evaluación y autoevaluación: Llevada por la Comision Ad Hoc para actualización de programas' compuesta por: 1 representante de coordinación de maestría clínica de la universidad, una curriculista y una funcionaria docente de institución 
formadora. Estas actividades se realizaron del 6 de marzo al 30 de septiembre de 2018, 52 reuniones, con 150 especialistas, donde participaron los responsables de los programas de los 5 hospitales formadores de la capital y los 7 hospitales de provincias, además de representantes de la sociedad médica correspondiente.

Cada hospital presentó las estrategias de implementación de su programa, las fortalezas y debilidades de su hospital, utilizando en el "formulario de reportes" que fue enviado con previamente al servicio formador, documento que contiene 9 temas basados en la guía para la acreditación de programas de residencias del Ministerio de Salud de Argentina, Postgraduate Medical Education, WFME Global Standards y el Consejo Americano para la Educación Médica Graduada, ACGME. Durante la presentación, se brindó asesoría y se entregó una plantilla única, como formulario oficial para los procesos de actualización de los programas académicos de las especialidades médicas.

Tercera: Asesoría para estructuración del programa. Simultáneamente se dió comunicación continua, vía correo electrónico o presencial, para revisión detallada del programa. Todos programas trabajaron activamente en la actualización.

Cuarta: Presentación y aprobación por entidades. Entre enero y octubre de 2018, se realizaron 3 presentaciones ante la junta de facultad de la Facultad de Medicina de la UP, aprobandose 16 programas de residencias como maestrías o doctorados que fueron: Radiología médica, Medicina Fisica y Rehabilitación, Medicina Preventiva (nuevo), Radio oncología (nuevo), Neumología, Anestesia y Reanimación, Hematología, Algiología. Medicina Interna, Medicina Familiar, Neurología (nuevo), Urología, Obstetricia Crítica, Geriatría, Nefrología y Cirugía Oral y Maxilofacial. Y se siguió trabajando en los programas faltantes, a mayo 2019 van 32 aprobados.

Quinta: Implementación: Se inicia simultáneamente con las anteriores, en junio 2018 se introducen para validación con nuevo grupo de 80 residentes, nuevos instrumentos de evaluación incluyendo el Portafolio o archivo de evidencias evidencia.

En agosto de 2018, se realiza el primer congreso de actualización médica para tutores clínicos, actividad en la cual participaron 200 médicos tutores del país y se irealiza el curso online de la Organización Panamericana de la Salud, con la participación de 60 tutores médicos, de agosto a diciembre.

Sexta: Evaluación y acreditación: No iniciada en 2018, se prepara para junio 2019, la evaluación del plan piloto de implementación de Portafolio, en 3 servicios médicos de un hospital formador.

Conclusión: Se diseña, inicia y formaliza con importantes resultados, el proceso de actualización universitaria, con fines de acreditación de los 52 programas de residencias médicas en la República de Panamá, con dinámica participación de todas las partes involucradas.

Hasta el momento el esfuerzo realizado ha sido una experiencia positiva para la universidad y las instituciones formadoras dada la enorme acogida de este proyecto que demuestra la importancia de la integración que debe darse entre los sistemas de salud, las universidades y los centros de investigación en términos de formación, actualización, innovación y educación médica continua que contribuirá a una mejor calidad de atención a la población.

\section{Evaluación deresidentes de pediatría mediante Mini-CEX en el primer nivel de atención}

Yulitta Horacio (1), Molina María (1), Maiolo Lucia (1), Paganini Agustina (1), Trini Julia (1)

(1)Dirección General de Docencia Investigación y Desarrollo Profesional. Ministerio de Salud. Ciudad Autónoma de Buenos Aires, Argentina.

Introducción: La residencia de pediatría es un modelo de formación profesional basado en la práctica clínica permanente y supervisada que requiere de un proceso de evaluación periódico.

El Mini Clinical Evaluation Exercise (Mini-CEX) se basa en la observación directa de la práctica médica con un perfil formativo que permite implementar estrategias docentes para mejorar el desempeño del residente. Esta metodología ha demostrado ser válida, fiable, factible, de fácil aplicabilidad, bajo costo y buena aceptación.

El propósito de este estudio es la implementación de esta herramienta de evaluación a residentes de segundo año, de pediatría, que se encuentran rotando en el primer nivel de atención, teniendo en cuenta las diferencias que plantea este escenario con respecto a otras áreas donde ya se ha implementado.

Métodos: Se realizó un estudio observacional y transversal. Se aplicó a residentes de segundo año de pediatría del Gobierno de la Ciudad de Buenos Aires que se encuentran rotando por los Centros de Salud y Acción Comunitaria (CeSAC), entre el 1 de Octubre de 2018 y el 30 de Abril de 2019. Los residentes que participaron del estudio provenían de Hospitales monovalente Pediátricos y de Hospitales Generales de Agudos. La estandarización de las evaluadoras se realizó mediante reuniones previas al inicio del proyecto donde se unificaron criterios de evaluación. Previo a la participación, se explicó en qué consistía el estudio y firmaron el consentimiento informado, para ser incluidos en la investigación. Se realizaron 2 observaciones de cada residente, con una diferencia de 15 días entre las mismas. 
Resultados: Se incluyeron dentro del estudio un total de 34 residentes, 22 provienen de Hospitales monovalentes Pediátricos y 12 de Hospitales Generales de Agudos. Se evaluaron 8 áreas por participante (Anamnesis, Habilidades de comunicación con el paciente y con los cuidadores primarios, Examen físico, Criterio Clínico, Tratamiento inicial, Profesionalismo y organización de la consulta). La escala de puntuación de las distintas áreas es de 1 a 6 , siendo 1 insatisfactorio y 6 muy por encima de las expectativas. Con respecto a las observaciones de hospitales pediátricos la valoración global total (promedio de las 8 áreas evaluadas) fue de 4,20, el área con mejor puntuación fue examen físico y la que presento dificultad fue tratamiento inicial. En cuanto a hospitales generales la valoración global total fue 4,13, el área con mejor puntuación fue profesionalismo y la que presento dificultad fue habilidades de la comunicación con niños y adultos por igual.

Conclusión: A partir de la aplicación del Mini-CEX en esta población se objetivaron las diferencias que presentan en la atención medica de pacientes los residentes de hospitales monovalentes pediátricos y Generales de Agudos en el área del primer nivel de atención y los aspectos de la formación de los mismos en los que se debe enfatizar, esta es una de las fortalezas de este método de evaluación. Las dificultades que encontramos en la implementación de la herramienta en el ámbito de rotaciones en centros de salud, fueron principalmente las vulnerabilidades sociales que presentan las familias y que implican una complejidad en las consultas a la que no están habituados los residentes, ya que no son de índole biomédica. Otra de las circunstancias es que la gran demanda asistencial y el volumen de residentes a supervisar dificultan resguardar el tiempo necesario sin interrupciones para llevar a delante la observación. En cuanto a la implementación en general de la herramienta, creemos que el principal inconveniente que puede surgir es la diferencia de criterios cuando son diferentes observadores para un mismo grupo, es por ello que hay que realizar la estandarización de las evaluadoras previo al uso de la herramienta de evaluación. No encontramos en el análisis de los datos diferencias significativas en la formación entre residentes de hospitales pediátricos y generales. Se realizará una devolución a las sedes formadoras sobre los resultados de la investigación para que puedan implementar un plan de mejoras tanto en tratamiento inicial como en habilidades de la comunicación.

\section{Programa de Recertificación del Consejo de Evaluación Profesional de la Sociedad Argentina de Pediatría: 14 años de Experiencia}

Yulitta Horacio (1), Pascual Carolina (1)

(1) Consejo de Evaluación Profesional. Sociedad Argentina de Pediatría (CEP)

Introducción: La revalidación debe entenderse como un proceso que, iniciado con la certificación, busca promover la inserción de los médicos especialistas en actividades de actualización permanente a lo largo de la vida profesional, que exija un esfuerzo posible cualesquiera sean las condiciones, el ámbito o la región en que cada profesional desarrolle su tarea.

Objetivo: Analizar los resultados obtenidos en base al uso de un instrumento inédito utilizado por el CEP para otorgar créditos para la recertificación.

Métodos: Se utilizó una herramienta construida por consenso entre expertos (miembros del Consejo de Evaluación Profesional (CEP) y la Secretaria de Educación continua de la SAP). La misma se utiliza desde el año 2004. Para revalidar se deben obtener 200 créditos en total ( 40 créditos por año revalidado). Se muestra a continuación resumen de la herramienta utilizada.

Área 1: Dedicación horaria asistencial semanal (50-100 créditos máximo)

Área 2: Actividades de Educación médica, docentes, académicas

a) Docencia

b) Congresos Actividad no SAP 50\%

c) Actividades de Educación médica continua o de actualización reconocidas por la SAP

d) Producción Científica

Área 3: Actividad Societaria

Área 4: Calidad de ejercicio profesional, resolución de dudas, aplicación de avances científicos, desarrollo de proyectos comunitarios.

Las áreas 2,3 y 4 permiten completar los créditos restantes

Versión completa y detalles de la herramienta en el link: https:// www.sap.org.ar/institucional/contenidos/5/cerfificacion-de-profesionales.html

Resultados: La SAP cuenta con 18000 socios de los cuales 8888 , obtuvieron una certificación. Están vigentes por el periodo de 5 años que dura la misma 3406. La certificación es voluntaria, así como la recertificación, y hasta el momento no es requisito para el ejercicio de la profesión, salvo en algunas de las 24 jurisdicciones del país.

\section{ARS MEDICA Revista de Ciencias Médicas}




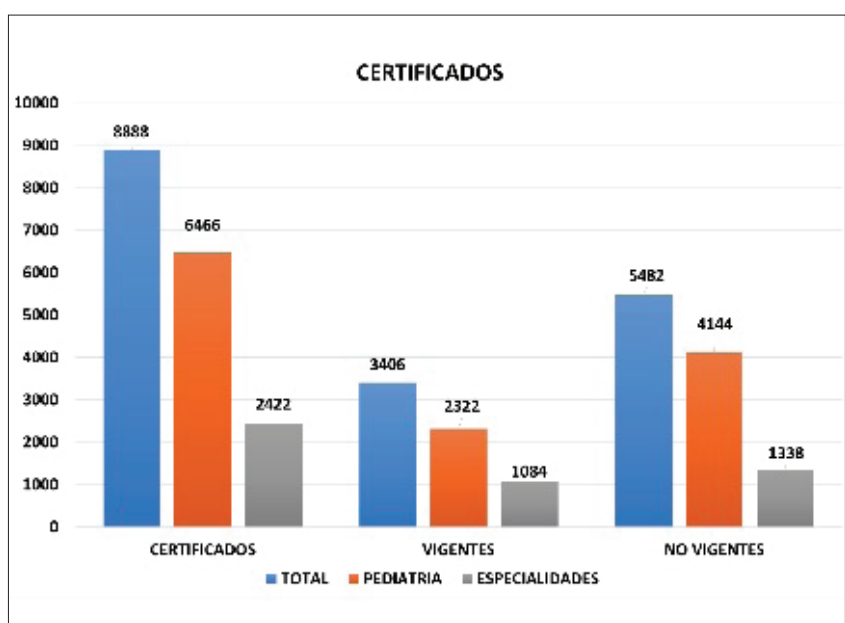

Conclusión: La Sociedad de Pediatría certifica pediatras desde el año 1968, el resto de las especialidades se fueron sumando progresivamente. A partir del año 2004, se inicia la recertificación, con el programa vigente, el mismo es utilizado por organismos de ley como una herramienta de calidad del ejercicio profesional. Existen pocos programas con estas características. En nuestro país es voluntario. Nuestra línea de trabajo está dirigida a la mejora de esta herramienta y a la adecuación de la misma a cada especialidad.

\section{Evaluación de impacto del Diplomado a distancia: Salud Integral de adolescentes. PUC/OPS}

Zubarew Tamara (1), Caffe Sonja (2), Rosenberg Hernan (2), Correa, Loreto (1), Inostroza Carolina (1), Urrejola Pascuala (1)

(1) Unidad de Adolescencia, Departamento de Pediatría. Pontificia Universidad Católica de Chile, (2) Organización Panamericana de la Salud, Washington DC. USA.

Introducción: En 2003 la Organización Panamericana de la Salud (OPS), Washington DC, estableció una alianza con la Unidad de Adolescencia de la División de Pediatría de la Pontificia Universidad Católica de Chile (PUC), para el desarrollo del Diplomado de educación a distancia "Desarrollo y Salud Integral del Adolescente". El curso consiste en un entrenamiento certificado modular de 9 meses, que se imparte a través de medios virtuales. Este Diplomado está basado en competencias y se caracteriza por alta interacción docente - estudiante, con el objetivo de entregar herramientas técnicas en el ám bito de promoción, prevención y tratamiento en atención de salud integral de adolescentes ${ }^{(1)}$.

En la literatura internacional hay controversia en cuanto al impacto del e-learning versus el aprendizaje presencial ${ }^{(2,3)}$.
Entre los años 2003 y 2017, la OPS ha otorgado becas de capacitación a profesionales de salud de diferentes países de América Latina y el Caribe.

La OPS está realizando una evaluación de la implementación del Plan Regional de Salud de Adolescentes, y dado que la capacitación de recursos humanos ha sido una de las áreas estratégicas de acción, se realizó el presente estudio en forma colaborativa con la PUC, con fin de revisar el impacto del Diplomado a distancia en el funcionamiento de los servicios de salud en América Latina. Este Diplomado es parte de la Beca de subespecialización en Medicina de Adolescentes de la PUC.

Objetivo: Evaluar el impacto del Diplomado de educación a distancia "Desarrollo y Salud Integral del Adolescente", en el desempeño profesional y funcionamiento de los servicios de salud de los participantes que han sido becados por la OPS.

Métodos: Estudio de tipo descriptivo. Se utilizó la base de datos de la PUC, con un registro total de 1200 participantes, de los cuales, entre los años 2003 y 2017, la OPS patrocinó 687 profesionales con becas, constituyendo el universo de evaluación. Se envió una encuesta vía correo electrónico, para estudiar el impacto del diplomado, la quefue contestada por un $41 \%$ de los participantes, cuyo perfil es muy semejante al universo total. Se analizó la percepción de los participantes, como resultado del Diplomado, en tres dimensiones, en escala de 1 a 10: a) Mejorías en competencias profesionales individuales: Se utilizaron las 17 competencias definidas por la OMS para la capacitación de proveedores de salud integral de adolescentes ${ }^{(4)}$, que incluyen ámbitos de salud y desarrollo, programas y políticas de salud, y habilidades de atención clínica en problemas de salud específicos. b) Mejorías en el trabajo: A través de la percepción del grado de avance de los participantes en atención de salud de adolescentes en cinco tipos de desempeño (Un cambio a un trabajo mejor, mayor autonomía, un aumento en la resolutividad, mayor satisfacción de los pacientes, y una mejoría en la carrera). c) Mejorías en el sistema de salud donde se desempeñan:Como resultado de la implementación de lo aprendido (ej. hacer el servicio más amigable para adolescentes).

Resultados: Los países participantes corresponden prioritariamente a países de LAC con menores índices de desarrollo (República Dominicana, Honduras y Panamá). Un $72 \%$ de los profesionales provienen de las ciencias médicas (medicina, enfermería, obstetricia), un $14 \%$ del área de sicología y un 14\% desde otras profesiones, incluyendo ciencias sociales, leyes y educación. Un $69 \%$ de ellos se desempeñan como proveedores de salud, un 17\% corresponden a autoridades sanitarias y un $2 \%$ viene del sector académico. Con respecto al análisis de la percepción de mejoría por parte de las participantes evaluadas en 3 dimensiones destaca lo siguiente: a)Mejorías de las competencias profesionales: Se obtuvo un promedio de 7, con un mayor puntaje en mejora de habilidades de interacción 
eficaz con adolescentes y entrega de servicios de prevención, detección, tratamiento y atención de problemas frecuentes de salud, incluido VIH y otros aspectos de salud sexual y reproductiva. Los menores impactos fueron en manejo de lesiones involuntarias y enfermedades crónicas. b) Mejorías en el trabajo: Se obtuvo un promedio mayor de 7.2, con los puntajes más altos en percepción de satisfacción de pacientes y capacidad de resolutividad. Hubo un menor aumento de la autonomía en el trabajo y cambios a un trabajo mejor, lo que refleja en parte la organización de los servicios de salud. c) Mejorías en el sistema de salud donde se desempeñan: Se usaron cuatro variables, con un nivel de satisfacción por encima de 7. Lo más relevante fue la mejoría en adherencia a las indicaciones por los adolescentes, mientras que el menor cambio se percibió en alineación de los servicios con las normas de atención de la OPS. La queja más frecuente se refiere a la dificultad de los sistemas para ajustarse a las técnicas aprendidas al volver los participantes al trabajo, así como la rigidez de los mismos.

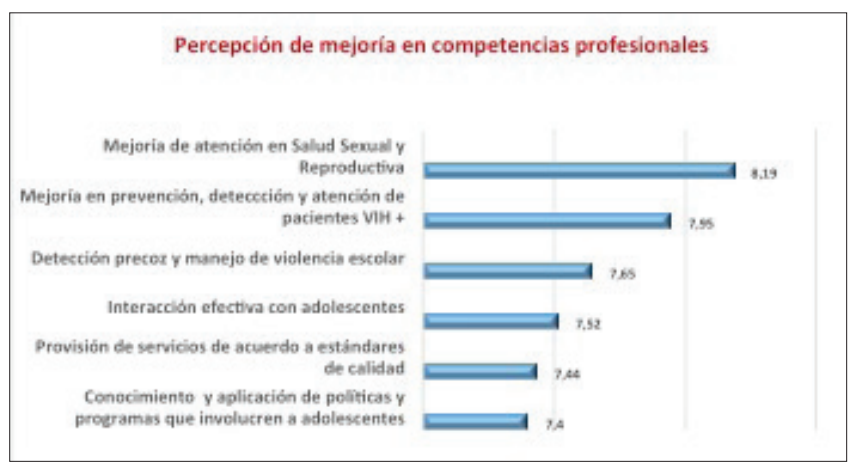

Figura 1.

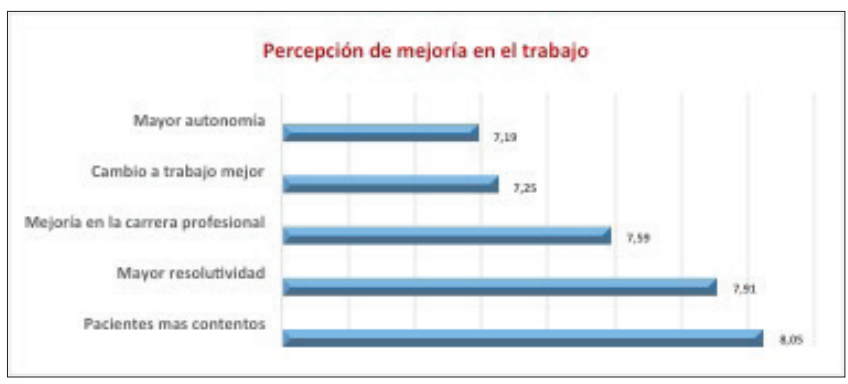

Figura 2.

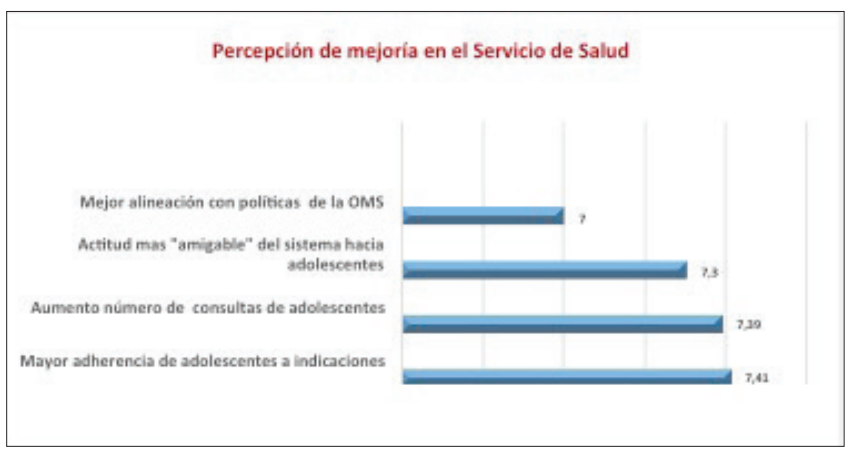

Figura 3.
Conclusión: Este estudio demuestra un alto impacto en adquisición de competencias a través de una estrategia de capacitación a distancia. Evidencia que la estrategia de aprendizaje utilizada en este diplomado de Salud de Adolescentes, caracterizada por alta interacción tutor-alumno y con énfasis en análisis de escenarios y casos clínicos, muestra mejorías en la adquisición de competencias y en el desempeño clínico de los profesionales, no sólo de manera individual, sino que también en mejoría en funcionamiento de los servicios de salud destinados a los adolescentes. Destaca la percepción del cambio hacia servicios de mejor calidad para adolescentes, reflejado en trato más empático, aumento de adherencia y mayor demanda de atención de salud. Además como resultado del diplomado se logra una mejoría en la situación laboral y profesional de los participantes.

\section{Bibliografía:}

ZubarewT. Educación médica a distancia: Diplomados on-line. Diploma de educación a distancia“"Desarrolloy Salud Integral del adolescente". ARS MEDICA Revista de Ciencias Médicas 2007; 36(2): 192-204.

Vaona A, Banzi R, Kwag KH, Rigon E, Cereda D, Pecoraro V, Tramacere I, Moja L. E-learning for health professionals. Cochrane Database of Systematic Reviews 2018, Issue 1.Art. No.:CD011736.

Rohwer A, Motaze NV, Rehfuess E, Young T. E-learning of evidence-based health care (EBHC) in healthcare professionals: a systematic review. Campbell Systematic Reviews 2017:4.

Organización Mundial de la Salud. Competencias básicas en materia de salud y desarrollo de los adolescentes para los proveedores de atención primaria. Organización Mundial de la Salud (2015). http:// www.who.int/iris/handle/10665/178251

Percepción Sobre la Retroalimentación Recibida Durante la Formación, Según Residentes y Egresados de Programas de Especialización en Psiquiatría de Chile

\section{Percepción Sobre la Retroalimentación Recibida Durante la Formación, Según Residentes y Egresados de Programas de Especialización en Psiquiatría de Chile}

Rubí Patricia (1), Bustos Claudio (1), Vergara-Barra Pablo (1), Rocha Marcela (1)

(1) Departamento de Psiquiatría y Salud Mental, Facultad de Medicina, Universidad de Concepción, Chile. 
Introducción: En Educación Médica, la retroalimentación constituye un instrumento que informa al estudiante sobre su proceso de aprendizaje y facilita los cambios necesarios ${ }^{1}$. Busca las propias percepciones del alumno respecto a su desempeño y el cómo mejorarlo, desarrollando habilidades de aprendizaje permanentes². Si se realiza de forma efectiva resulta útil para mejorar el rendimiento, las habilidades clínicas y de comunicación, y el trato hacia los pacientes ${ }^{3}$. Si se entrega de mala forma produce desmotivación y un quiebre en la relación docente-alumno ${ }^{4}$.

Tal como sucede en pregrado, la formación de especialistas ocurre en su mayoría en situaciones clínicas, por lo que la retroalimentación es un componente crucial para el aprendizaje en estos contextos $^{5}$. Sin embargo, estudios muestran que un alto porcentaje de residentes refiere no haberla recibido nunca o de manera infrecuente ${ }^{6}$. El objetivo del presente trabajo fue evaluar la percepción de los residentes de psiquiatría de Chile sobre la retroalimentación recibida durante su formación.

Método: Diseño observacional, correlacional, transversal. Se aplicó una encuesta en línea a una muestra intencionada de 87 residentes de Chile. Se contactó a los diferentes jefes de programas de especialización, quienes facilitaron los correos electrónicos de sus residentes. Respondieron la encuesta 51 mujeres y 36 hombres, con una media de edad de 31,1 años. 76 residentes se encontraban cursando programas de especialización en Psiquiatría del Adulto, 10 en programas de Psiquiatría Infanto-Juvenil y 1 en un programa de especialización en Psiquiatría Forense, de 12 centros formadores. Se enviaron invitaciones para responder a todos éstos, en noviembre de 2016 y junio de 2018.

Resultados: La mediana en la frecuencia en que los residentes han recibido retroalimentación de parte de sus docentes fue de 3 en una escala de 1 (nunca) a 5 (muchas veces) (Figura №1), específicamente retroalimentación positiva con una mediana de 4 y retroalimentación negativa de 3 . Esta retroalimentación ha sido recibida frente a otros con una mediana de frecuencia de 3 , principalmente frente a otros residentes. El 64\% afirmó que su programa de especialización cuenta con una instancia formal de entrega de retroalimentación y la mediana de satisfacción con ésta se encuentra en la categoría "satisfecho". La mediana sobre cuánto la retroalimentación ha influido en la motivación al aprendizaje fue de 4 en una escala de 1 (nada) a 5 (mucho). Las actividades mencionadas como más retroalimentadas fueron: la entrevista clínica y confección de historia clínica, ambas con una mediana de 4 en una escala de 1 (nunca) a 5 (muchas veces). En relación a las características de la retroalimentación recibida: respeto, atingencia y claridad, la mediana fue de 4 en una escala de 1 (pésimo) a 5 (óptimo). El 39\% de los residentes consideró que la retroalimentación negativa mejora la relación con el docente que la entrega. El 40\% de los residentes busca activamente ser retroalimentado y el 54\% no la busca pero la acepta de buena manera. $89 \%$ señaló que la retroalimentación es muy importante en la formación de psiquiatras y el 65\% afirmó que fue recibida de los docentes a los que considera más cercanos.

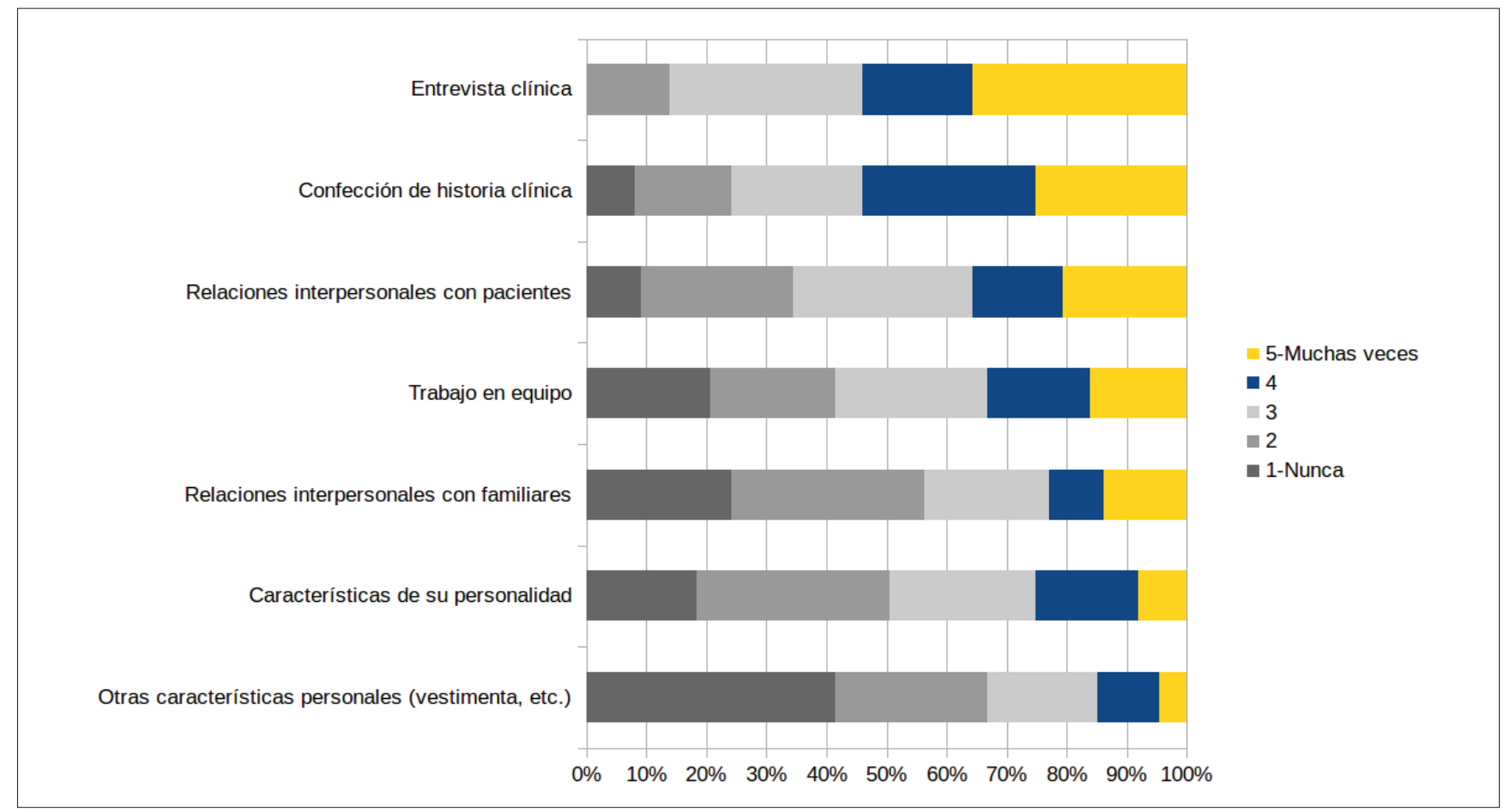

Figura N¹: Frecuencia y escenarios en los que los residentes han recibido retroalimentación. 
Conclusión: La retroalimentación cumple un rol moderador significativo en la formación de los residentes de psiquiatría, siendo considerada por ellos una actividad muy importante, que aporta y motiva su aprendizaje. La mayoría de los centros formadores cuenta con instancias formales de retroalimentación, entregándose ésta con respeto, de manera atingente y clara.

\section{Referencias:}

Ramaprasad A. On the definition of feedback. Behav Sci. 1983; 28:4-13.

Brown N, Cooke L. Giving effective feedback to psychiatric trainees. Adv Psychiatr Treat. 2009; 15: 123-128.

Salam A, Halizah H, Mohamad N, Das S, Yousuf R. Bedside teaching in undergraduate medical education: Issues, strategies, and new models for better preparation of new generation doctors. Iran J Med Sci. 2011; 36(1): 1-6.

Kluger AN, DeNisi A. The effects of feedback interventions on performance: A historical review, a meta-analysis, and a preliminary feedback intervention theory. Psychol Bull. 1996; 119(2): 254-284.

Archer JC. State of the science in health professional education: effective feedback. Med Educ. 2010; 44: 101-108.

Isaacson J, Posk L, Liaker D, Halperin A. Resident perceptions of the evaluative process. J Gen Intern Med. 1995;10 (suppl): 89.

\section{¿Qué significa ser un educador médico?: Una comparación binacional entre Canadáy México}

Olivares Silvia (1), López Mildred (1), Turrubiates Miriam (1), Steinert Yvonne (2)

(1) Tecnológico de Monterrey, Escuela de Medicina y Ciencias de la Salud, (2) McGill University, Montreal, Canadá

Introducción: La identidad profesional de los educadores médicos describe la construcción y organización de la comprensión de sí mismos y su papel como facultad. Esta identidad debe ser congruente con los procesos en los que los individuos desarrollan sus comportamientos, ideas, preferencias, aspiraciones y estilo de enseñanza. Permitiendo replantear el concepto de "trabajar como miembro de la facultad" hacia "ser un educador médico". El objetivo de este estudio fue explorar y comparar cómo los docentes de dos universidades integran su función docente en su identidad profesional.

Método: Se realizó un estudio cualitativo que involucró a 26 educadores médicos en una escuela de medicina canadiense (13) y una mexicana (13). Los participantes fueron entrevistados con la pregunta: ¿qué significa ser un educador médico? y sus narrativas se codificaron utilizando Kegan (1982) etapas de desarrollo de la identidad profesional: 1) imperial, 2) interpersonal, y 3) institucional. La etapa imperial tiene en cuenta la visión de los demás, pero predominan los intereses propios. Interpersonal cuando el individuo puede ver múltiples perspectivas y le preocupa la percepción de los demás. Institucional es un individuo que puede asumir un papel y establecer relaciones mientras lo evalúa en términos de principios y estándares autoproclamados independientemente de los demás.

Resultados: La distribución de respuestas de educadores clínicos mexicanos fue: $46 \%$ imperial, 38\% interpersonal, 15\% institucional. Los profesores canadienses fueron codificados como: $15 \%$ imperial, $46 \%$ interpersonal y $38 \%$ institucional. La formación de identidad en los participantes canadienses se encuentra en niveles de etapa más avanzada que los mexicanos.

Conclusión: Las primeras etapas están relacionadas con el autoconocimiento del individuo que crea sus propias creencias y valores. Estos valores están moldeados por las experiencias con otros como miembros de un equipo en la etapa interpersonal, como en el proceso de enseñanza y aprendizaje. Sin embargo, para poder alcanzar niveles más altos, la persona necesita liderar iniciativas para que otros los ayuden a crear su identidad.

El proceso de formación de identidad comienza desde la participación periférica hasta la participación plena a través de la interacción social en las comunidades de práctica. Con el fin de mejorar la formación de la identidad de los educadores médicos es importante facilitar el desarrollo de la facultad con proyectos de transformación donde pueden interactuar simultáneamente con los estudiantes y compañeros.

\section{Diseño e Implementación de una Herramienta Electrónica de Registro en Smartphone para Optimizar la Vigilancia de la Morbilidad Postoperatoria en un Departamento Quirúrgico de un Hospital Universitario}

\author{
Torregrosa Lilian (1), Ariza Aníbal (2), Aponte Andrés (3)
}

(1) Departamento de Cirugía General Hospital Universitario San Ignacio, Bogotá, Colombia, (2)Cirugía General, Pontificia Universidad Javeriana, Bogotá, Colombia.

Introducción: Las complicaciones postoperatorias son una causa importante de mortalidad y de discapacidad a nivel mundial. Si 
bien, la presencia de las mismas, hacen parte inherente de un procedimiento quirúrgico, su evaluación y análisis en un Servicio de Cirugía General constituye la herramienta más importante para la prevención y el adecuado manejo de las mismas.

En nuestra experiencia a nivel local, existe una gran inconsistencia en la forma en que se documentan las complicaciones. La renuencia a reportar resultados negativos y las preocupaciones médico-legales, han perjudicado la capacidad de los departamentos de cirugía para recopilar los datos de complicaciones, necesarios para identificar las áreas susceptibles de procesos de mejoramiento en calidad.

En nuestro departamento de cirugía, hemos optado por diseñar esta herramienta, para fomentar el registro oportuno de las complicaciones, y crear una cultura en nuestros residentes, de ser capaces de asumir con autocritica la presencia de estos eventos y a partir de un análisis detallado implementar acciones de mejora en cada uno de los casos. Adicionalmente, tener la información completa y precisa de estos eventos, nos permitiría como departamento quirúrgico, evaluar nuestros resultados y saber con precisión nuestros indicadores de calidad en cuanto a tasas de ISO, re intervención no programada, infecciones asociadas al cuidado de la salud, eventos tromboembolicos, etc

\section{Métodos:}

1. Análisis del problema y revisión de literatura

2. Diseño de sistema de registro (Google Forms - Acceso fácil en Smartphone)

3. Recolección de datos de complicaciones postoperatorias durante la valoración diaria de pacientes (hospitalización, urgencias, consulta externa)

4. Análisis crítico e individual del evento por el residente a cargo (Reporte del análisis con datos disponibles en la literatura en relación con la complicación, determinar frecuencia, factores predisponentes, definir si fue un evento prevenible o no, acciones inseguras, intervenciones requeridas, consecuencias para el paciente, etc.)

5. Análisis mensual de los resultados registrados en junta general del departamento.

Resultados: Los resultados en el periodo de tiempo comprendido entre 04/17 hasta 01/19 de registro, fueron 456 registros de complicaciones en 8691 procedimientos realizados. La gran mayoría de estos eventos, pertenencias a la clasificación I-II de Clavien Dindo (CD), 42\% requirieron algún tipo de intervención quirúrgica, endoscópica o percutánea (CD III) y un 2\% requirió manejo en UCI (CD IV).

Las complicaciones se presentaron de forma equitativa en la población masculina y femenina, y como es de esperarse fueron más frecuentes en población mayor de 65 años con ASA III-IV.
En particular, se disminuyó el subregistro de ISO, en nuestro registro obtuvimos 112 casos de nuestro departamento, en comparación con 76 reportados por nuestro hospital en todas las especialidades.

Conclusión: Crear la cultura del registro y el análisis de complicaciones postoperatorias debe ser un esfuerzo de todos los programas de cirugía general; es nuestra responsabilidad como docentes, establecer ambientes propicios para la evaluación de estos eventos desde la academia e incentivar a nuestros residentes a afrontar las complicaciones como situaciones esperables en cirugía, pero que requieren un proceso de evaluación autocritica para evitar su aparición y sus consecuencias en los pacientes.

\section{Aptitud clínica final en internado de pregrado en sedes hospitalarias de Nuevo León}

Cobos-Aguilar Héctor (1), Pérez-Cortés Patricia (1), MartínezDelgado Guillermo (1), Hernández-Portales Jorge (1), MendozaVillalba Jaziel (2), Gómez-García Salvador (2), Jacobo-Saucedo Luis (3)

(1) Universidad de Monterrey, N.L., México, (2) IMSS, HGZ 2, HGZ 4, N.L., México, (3)Secretaría de Salud, N.L., México

Introducción: La evaluación del aprendizaje clínico se indaga con diversos instrumentos, como los casos clínicos reales problematizados que indagan la aptitud clínica (APCL) que se define como la capacidad del médico para decidir entre diferentes opciones, la más adecuada, en un caso particular, con sus diferentes indicadores: factores de riesgo (FR), diagnóstico (DX), uso de paraclínicos $(\mathrm{PCL})$, tratamiento $(\mathrm{TX})$, pronóstico (PRN). En México los resultados han sido bajos en médicos internos de pregrado (MIP). Por ello el objetivo fue comparar la APCL final del IP de cinco hospitales de Nuevo León.

Métodos: Se estudiaron 70 médicos MIP en una muestra censal. Se aplicó un instrumento al final del internado, válido y consistente (KR: 0.77) con 30 casos clínicos, que exploraban padecimientos más frecuentes en los hospitales (apendicitis aguda, preclamsia, neumonía, infarto agudo al miocardio, diabetes mellitus, etc.), con 5 indicadores: FR, DX, PCL, TRAT y PRN, de medicina interna (MI), pediatría (PED), medicina familiar (MF), urgencias (URG), cirugía general (CG) y ginecoobstetricia (GO). Fueron 75 reactivos por módulo, 450 en total. Se analizó promedio escolar, medianas por módulo y universidad de procedencia. Estadísticos: Kruskal-Wallis, U de Mann-Whitney, Rho de Spearman (medianas entre sedes por indicador y universidad) promedio vs. mediana global). 
Resultados: No hubo correlación entre promedio y mediana global. Se observó una APCL baja en las sedes (Mediana global: 177/450). Se observó $p<0.05$ a favor de $G_{1}$ y $G_{5}$ en casi todos los módulos e indicadores vs. $\mathrm{G}_{4}$ Las medianas más altas se obtuvieron en URG (50/75) y MF (43/75) y las más bajas en GO (5/75). Los aciertos más altos se observaron en PRN (48\%) y DX (41\%), el más bajo en TRAT (33\%), $\mathrm{p}=\mathrm{NS}$ en FR y PCL. Entre universidades $\mathrm{p}=\mathrm{NS}$ en $\mathrm{MI}, \mathrm{CG}, \mathrm{FR}$, PCL y PRN. En 22 CC se obtuvieron menos del $50 \%$ de respuestas correctas y de éstos, cuatro estuvieron por debajo del 15\% (apendicitis aguda, preeclampsia, endometriosis y pielonefritis). Ocho estuvieron entre $51 \%$ y $60 \%$ y tres entre $62 \%$ y $73 \%$ IAM y EHH en URG, y $\mathrm{DM}_{2}$ en MF.

Conclusión: La APCL debe fortalecerse en los MIP en GyO y en DX, aún insuficientes. El instrumento fue elaborado por MIP recién egresados por se esperaban mejores resultados. No se relacionaron los resultados con los ambientes educativos, lo que consideramos indispensable para evaluar en forma integral el desarrollo de esta relevante habilidad médica. La comunicación de los resultados de evaluación debe ser constante entre las universidades y los hospitales sedes para fortalecerla.

\section{Enseñanza y Evaluación del Rol Académico CanMEDS en Residentes de Especialidad y Subespecialidad Médica de la Pontificia Universidad Católica de Chile}

Basauri Sofía (1), Riquelme Catalina (2), Peña, Arturo (1), Navea Óscar (1), Gonzále Matías (3), Cifuentes Lorena (2), Hoyl Trinidad (2)

(1)Sección Medicina de Urgencia. Escuela de Medicina, (2)Dirección de Postgrado. Escuela de Medicina, (3)Centro de Edución Médica y Ciencias de la Salud. Escuela de Medicina, Pontificia Universidad Católica de Chile, Santiago, Chile.

Introducción: Para ser un Médico Experto, de acuerdo al Modelo de Competencias CanMEDS, los residentes deben desarrollar Roles o meta-competencias que les permitan desempeñarse efectivamente en su práctica médica. Uno de ellos es el Rol Académico, para el cual es necesario desarrollar e implementar instancias de enseñanza y evaluación que promuevan el aprendizaje continuo y la docencia en los residentes, como parte de su formación profesional. Es por este motivo, que se propuso como objetivo diseñar e implementar un curso en metodología b-learning (semi-presencial), dirigido a residentes de la Escuela de Medicina UC, para enseñar y evaluar el Rol Académico CanMEDS.

Métodos: Para crear el curso, se llevó a cabo un proceso estandarizado, que incluyó las siguientes etapas: diseño, producción, implementación y evaluación. En la etapa de diseño se identificaron las competencias del Rol que se abordarían en el cuso y se realizó el diseño instruccional (se definieron objetivos de aprendizaje, contenidos, metodología y evaluaciones). Durante la producción, se desarrolló el material pedagógico para las sesiones online y talleres presenciales. Además se realizó la grabación y edición de los videos de las clases online. La etapa de implementación se realizó a través de un sistema de tutoría administrativa para la gestión de la plataforma online y comunicación con los residentes, y una tutoría académica, para brindar retroalimentación a las evaluaciones. En la etapa de evaluación se aplicó la Encuesta de Satisfacción del curso a los residentes, a partir de la cual se fueron realizando los cambios y reediciones correspondientes.

Resultados: Se desarrolló un curso en metodología b-learning (7 sesiones online y 3 talleres presenciales) dirigido a residentes de especialidades y subespecialidades médicas de la Escuela de Medicina UC. El curso entregó conceptos básicos y herramientas útiles para promover en los residentes el desarrollo de competencias asociadas al aprendizaje continuo y la enseñanza a otros, tales como: técnicas de docencia bedside, clase invertida, presentaciones efectivas, simulación y feedback, entre otros.

La evaluación se realizó a través de tareas semanales y actividades en talleres presenciales, con el objetivo de promover la práctica reflexiva sobre sus propias experiencias como alumnos y docentes en la práctica diaria, y aplicar las herramientas aprendidas, facilitando su rol como docentes. Se buscó incentivar a los residentes a desarrollar sus competencias a través de la propuesta de nuevas ideas para mejorar su desarrollo académico personal y en su programa de residencia.

El curso se dictó por primera vez en metodología b-learning en el año 2016, como parte de los cursos transversales para residentes. Posteriormente, se realizaron dos versiones en el año 2017, y dos en el año 2018, con un total de 144 estudiantes en todas las versiones (117 pertenecientes a especialidades médicas, 25 a susbepecialidades médicas, 1 residente de odontología y 1 estudiante del Magíster en Educación Médica). A través de una Encuesta de Satisfacción, utilizando una escala tipo Likert, 112 (77,8\%) residentes evaluaron el curso. En cuanto al contenido, un $84,8 \%$ dice estar muy de acuerdo y un $15,2 \%$ de acuerdo con que los contenidos y conceptos del curso les han permitido aprender sobre nuevas herramientas de enseñanza en educación médica que serán de utilidad para ejercer su rol como docente. Un $81,3 \%$ de los residentes refiere estar muy de acuerdo con que el curso les ha permitido reflexionar y tomar conciencia sobre su rol como académico en su desempeño cotidiano. Con respecto a la metodología b-learning, un 78,6\% está muy de acuerdo y un $18,8 \%$ de acuerdo con que esta metodología les acomoda más que la presencial. Finalmente, con respecto a la pregunta de si recomendaría este curso a otros colegas, $83,9 \%$ de los residentes dice estar muy de acuerdo y $16,1 \%$ de acuerdo con esta afirmación. 
Al codificar los comentarios de las encuestas realizadas, surgieron categorías relacionadas con la alta calidad del curso, siendo este valorado por los contenidos entregados y la disposición y preparación del equipo docente. Asimismo, los residentes mencionan que el curso les entregó los contenidos y herramientas para ejercer su rol como docente en la práctica diaria en la interacción con internos, otros residentes y pacientes. Sugieren como cambios para mejorar el curso, realizar modificaciones a la extensión de las clases videadas y tareas, así como también a la metodología utilizada en algunas de las evaluaciones.

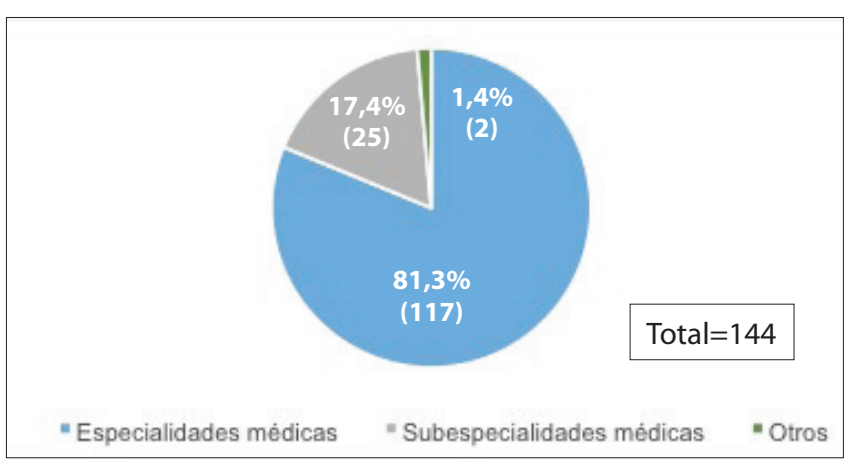

Figura 1. Total de residentes que han realizado el curso, según especialidad y subespecialidad médica.

Tabla 1. Especialidades y Subespecialidad Médicas a las cuales pertenecen los residentes que realizaron el curso.

\begin{tabular}{l|l}
\hline Especialidades Médicas & Subespecialidades Médicas \\
\hline & \\
Anestesiología & Cardiología Pediátrica \\
Anatomía Patológica & Cirugía Cardiovascular \\
Cirugía & Endocrinología Adulto \\
Dermatología & Enfermedades Infecciosas del Adulto \\
Medicina Familiar Mención Adulto & Enfermedades Respiratorias del \\
Medicina Familiar Mención Niño & Adulto \\
Medicina Interna & Enfermedades Respiratorias del Niño \\
Medicina de Urgencia & Enfermedades Respiratorias y Me- \\
Neonatología & dicina Intensiva del Adulto \\
Neurocirugía & Gastroenterología \\
Neurología & Geriatría \\
Neurología Pediátrica & Hematología y Oncología Pediátrica \\
Nutrición Clínica y Diabetología & Inmunología Clínica y Reumatología \\
Obstetricia y Ginecología & Inmunología Clínica yeumatología \\
Otorrinolaringología & Pediátrica \\
Pediatría & Medicina Intensiva del Adulto \\
Psiquiatría & Medicina Intensiva del Niño \\
Psiquiatría del Niñoy del Adolescente & Nefrología \\
Radiología & Nefrología Pediátrica \\
Traumatología y Ortopedia & Nutrición Clínica y Diabetología \\
Urología & Psiquiatría de Enlace y Medicina \\
& Psicosomática \\
\hline & \\
\hline
\end{tabular}

Tabla 2. Principales resultados de la Encuesta de Satisfacción del curso, de acuerdo a una escala Likert de 1 (muy en desacuerdo) a 4 (muy de acuerdo) para las siguientes afirmaciones.

\begin{tabular}{|c|c|c|c|c|}
\hline Ítem & $\begin{array}{l}\text { Muy en } \\
\text { desacuerdo }\end{array}$ & $\begin{array}{l}\text { En } \\
\text { desacuerdo }\end{array}$ & $\begin{array}{l}\text { De } \\
\text { acuerdo }\end{array}$ & $\begin{array}{l}\text { Muy de } \\
\text { acuerdo }\end{array}$ \\
\hline $\begin{array}{l}\text { Los contenidos } \\
\text { y conceptos del } \\
\text { curso me han per- } \\
\text { mitido aprender } \\
\text { sobre nuevas } \\
\text { herramientas de } \\
\text { enseñanza en } \\
\text { educación mé- } \\
\text { dica, que serán } \\
\text { de utilidad para } \\
\text { ejercer mi rol } \\
\text { como docente. }\end{array}$ & & & $\begin{array}{c}15.2 \% \\
(17)\end{array}$ & $\begin{array}{c}\mathbf{8 4 . 8 \%} \\
(95)\end{array}$ \\
\hline $\begin{array}{l}\text { Los contenidos } \\
\text { del curso son } \\
\text { aplicables a mi } \\
\text { trabajo y espe- } \\
\text { cialidad. }\end{array}$ & & $\begin{array}{l}1.8 \% \\
(2)\end{array}$ & $\begin{array}{c}19.6 \% \\
(22)\end{array}$ & $\begin{array}{c}78.6 \% \\
(88)\end{array}$ \\
\hline $\begin{array}{l}\text { El curso me ha } \\
\text { permitido re- } \\
\text { flexionary tomar } \\
\text { conciencia sobre } \\
\text { mi Rol Académico } \\
\text { en mi desempeño } \\
\text { cotidiano. }\end{array}$ & & $\begin{array}{c}\mathbf{0 . 9 \%} \\
(1)\end{array}$ & $\begin{array}{c}17.9 \% \\
(20)\end{array}$ & $\begin{array}{c}81.3 \% \\
(91)\end{array}$ \\
\hline $\begin{array}{l}\text { El sistema b-lear- } \\
\text { ning meacomoda } \\
\text { más que el siste- } \\
\text { ma presencial. }\end{array}$ & & $\begin{array}{c}2.7 \% \\
(3)\end{array}$ & $\begin{array}{c}\mathbf{1 8 . 8} \% \\
(21)\end{array}$ & $\begin{array}{c}78.6 \% \\
(88)\end{array}$ \\
\hline $\begin{array}{l}\text { Recomendaría el } \\
\text { curso de todas } \\
\text { maneras a otros } \\
\text { colegas. }\end{array}$ & & & $\begin{array}{c}16.1 \% \\
(18)\end{array}$ & $\begin{array}{l}83.9 \% \\
(94)\end{array}$ \\
\hline
\end{tabular}

Conclusión: La enseñanza y evaluación del Rol Académico es fundamental para que los residentes adquieran las competencias necesarias para convertirse en un Médico Experto. A través de un curso formal, se muestra una manera efectiva para cumplir con este objetivo, promoviendo en los residentes una práctica reflexiva y entregándoles herramientas útiles para realizar docencia en su práctica cotidiana. Asimismo, ellos valoran esta oportunidad de aprendizaje, mostrándose satisfechos con los contenidos entregados y la metodología utilizada.

\section{ARS MEDICA Revista de Ciencias Médicas}




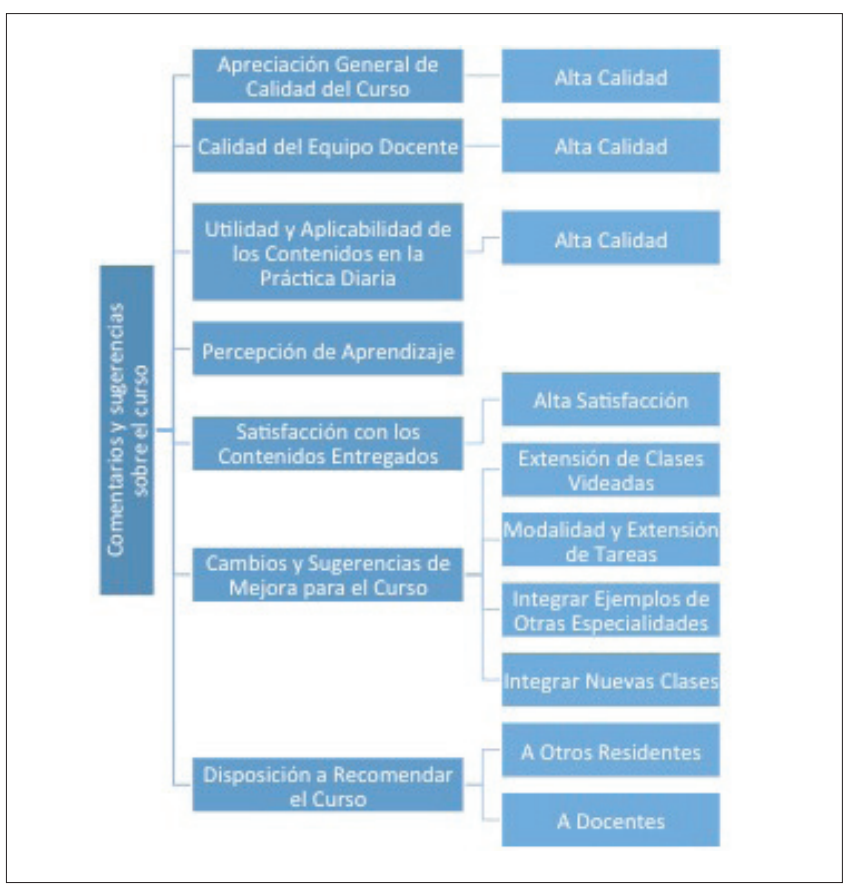

Figura 2. Análisis cualitativo de los comentarios de la Encuesta de Satisfacción del curso.

\section{Alumnos tutores en lectura crítica de investigación en internado de pregrado en Nuevo León. Experiencia de 7 años}

Cobos-Aguilar Héctor (1), Pérez-Cortés Patricia (1), HernándezPortales Jorge (1) Gómez-García Salvador (2), Romero-García Miguel (3)

(1) Universidad de Monterrey, N.L., México, (2)IMSS, HGZ 4, Guadalupe, N.L., México, (3)IMSS, HGZ 33, Guadalupe, N.L., México.

Introducción: La investigación es esencial en salud pero su aprendizaje está poco desarrollado en pregrado, incluyendo médicos internos de pregrado (MIP) especialidades y subespecialidades médicas. La lectura crítica de la investigación (LCR) promueve su aprendizaje pero es una habilidad metodológica tan compleja como la habilidad docente que apoyan la clínica para una formación integral. El peer-teaching learning (PAL) puede ser una respuesta integradora en los diferentes programas educativos. EI PAL se ha implementado en MIP con alumnos tutores (AT) desde hace 7 años. El objetivo del estudio fue referir la experiencia en 7 años de cursos de LCR por PAL (2011- 2018).

Métodos: Participaron 22 AT y 271 MIP en cinco cursos y 15 subgrupos. Se seleccionaron los AT por completar un curso previo de LCR, asistencia completa, elaboración de tareas, resultado >P90 en instrumento final y aceptación voluntaria. Condujeron un curso de 40 horas, semanalmente, a MIP, quienes criticaban artículos y resolvían guías de lectura con aspectos metodológicos y estadísticos pertinentes y discusión grupal coordinada por los AT. Se utilizó un instrumento válido y confiable (KR: 0.74) al inicio y final de cada curso, de 96 reactivos, 32 por indicador (interpretar, enjuiciar, proponer y global), 12 por diseño (casos y controles, cohortes, ECA, pruebas diagnósticas, encuestas, metaanálisis, instrumentos y supervivencia) y 48 respuestas correctas e incorrectas del tipo C, F, No Sé. La unidad de análisis fue la mediana. Estadísticos: Wilcoxon para antes y después, avance ponderal y determinación del azar en cada grupo.

Resultados: Se eliminaron 29 MIP por inasistencias, instrumento evaluado solo al inicio o al término. Los 15 grupos avanzaron (excepto tres), significativamente. La mediana global aumentó 12 vs. 25 y el azar disminuyó 0.67 vs. 0.25 antes y después y un avance ponderal grupal (2.54).

Conclusión: Los AT pueden ser excelentes docentes en aspectos que se consideran solo de habilidad en posgrado. La relación que se establece es muy cercana y los resultados esperanzadores. La disponibilidad de autoridades interinstitucionales es meritoria.

\section{Evaluación de dos competencias en Anestesiología (Cuidado del paciente y mejoramiento de la calidad basado en la práctica): una perspectiva desde los residentes, docentes y jefes de programa}

Jaramillo Sandra (1), Durante Eduardo (2), Ladenheim Roberta (2), Diaz Juan (1)

(1) Clínica de Marly, Universidad de los Andes, Colombia, (2) Instituto Universitario Hospital Italiano de Buenos Aires, Argentina.

Introducción: Estudios muestran el desequilibrio a la hora de evaluar el desempeño por competencias en anestesiología (marco teórico de la ACGME), inclinando la balanza a estrategias de evaluación ligadas a las competencias "cuidado del paciente (CP)" y "conocimiento médico (CM)", mientras que competencias como "aprendizaje autodirigido y mejoramiento basado en la práctica $(\mathrm{MBP})$ " raramente son abordadas. Tratando de responder a la pregunta: ¿Cuál es la percepción que tienen residentes, docentes y jefes de programa en relación con las estrategias de evaluación utilizadas en los programas de formación en anestesiología en Colombia?, este trabajo se propuso explorar estas percepciones en dos competencias trazadoras: cuidado del paciente y mejoramiento de la calidad basado en la práctica. 
Métodos: Se desarrollaron y aplicaron a los programas de formación en anestesia acreditados en Colombia, tres instrumentos para ser resueltos de manera virtual por directores de programa, residentes y docentes, previa aprobación del comité de ética. Para el análisis de datos se utilizó el paquete estadístico SPSS 17; se buscaron diferencias entre las percepciones de los docentes y los residentes en las variables que lo permitieron por medio de la prueba $\mathrm{X} 2$ y el test de Tau-b de Kendall.

Resultados: Se obtuvo una tasa de respuesta por programas del 75\% (15/20), 72(58\%, TR:29.7\%) respuestas correspondieron a residentes y 52 a docentes (42\%, TR:27.4). 2(13\%) programas de los encuestados manifestaron tener un currículo por competencias, el $100 \%$ de los programas evaluaban las 6 competencias genéricas de la ACGME. Las percepciones sobre la evaluación del CP no mostraron una distribución simétrica entre docentes y residentes. El tipo de test de evaluación más utilizado para valorar el CP fueron los test de conocimientos para los residentes $(73,54 \%$ ) y los test de contexto clínico para los docentes (85,3\%). Se encontraron diferencias significativas entre la percepción de docentes y residentes en los 14 hitos de esta competencia. La competencia $M B P$, aunque mostro un patrón de distribución simétrico, evidenció una tendencia por la utilización de test de contexto clínico en los residentes $(63,1 \%)$ y los test de evaluación práctica en los docentes (59,6\%). Se encontraron diferencias significativas en los hitos "Incorporación de iniciativas de calidad" $(p<0,05)$ y "Aprendizaje autodirigido" $(p<0,01)$.Gráficos 1 y 2

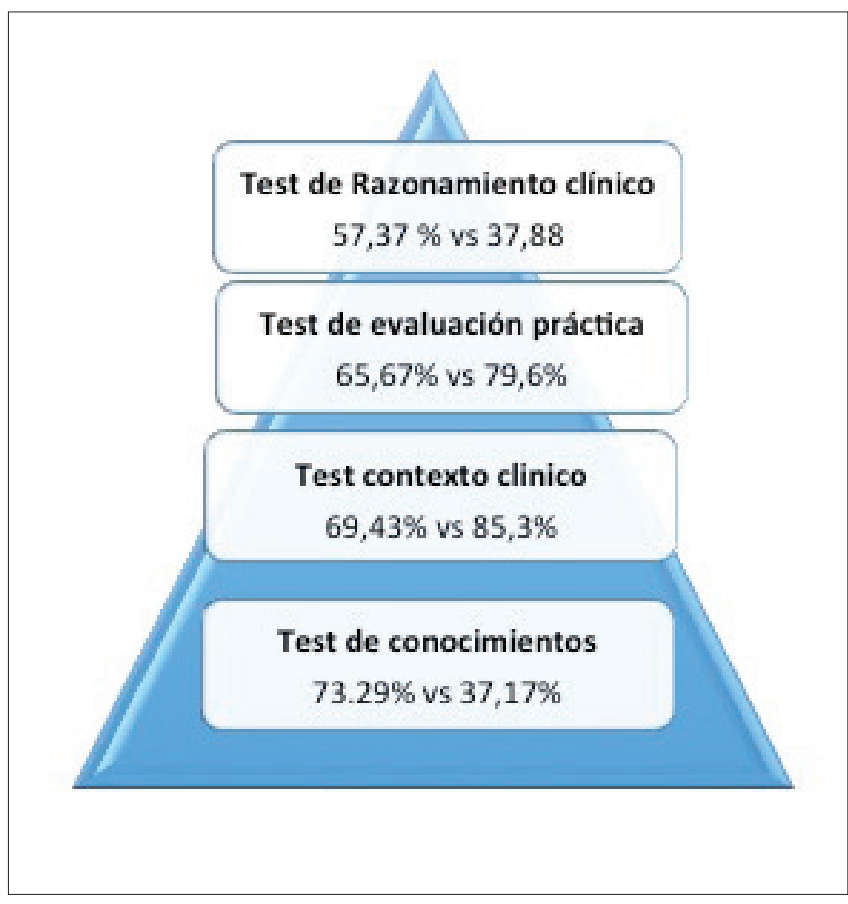

Gráfico 1: Frecuencia de utilización (Frecuente/muy frecuente) de las herramientas de evaluación de la competencia: Cuidado del paciente según residentes vs docentes

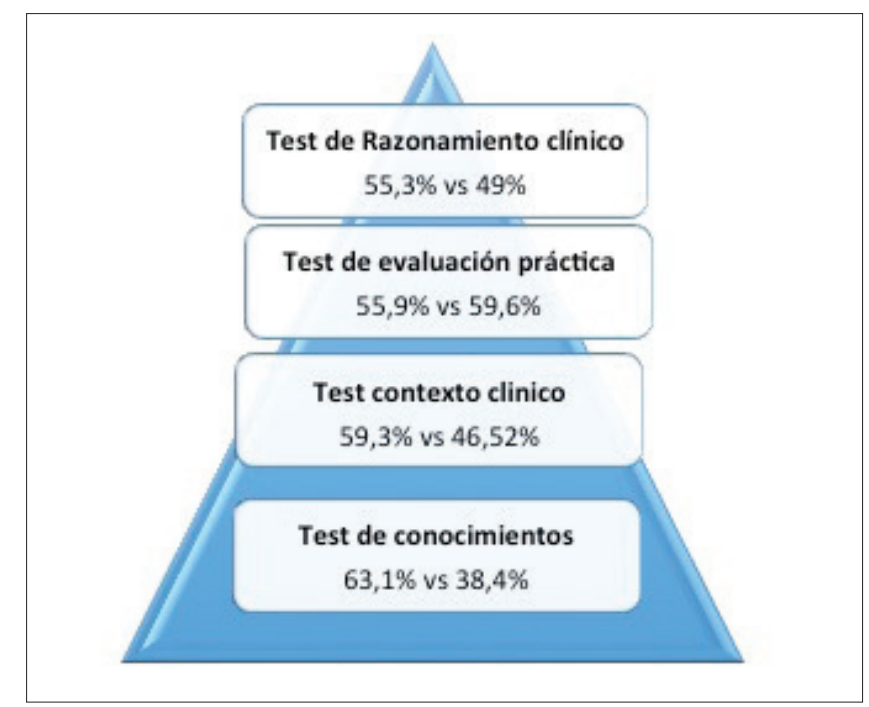

Gráfico 2: Frecuencia de utilización (Frecuente/muy frecuente) de las herramientas de evaluación de la competencia: Mejoramiento de la calidad basado en la práctica según residentes vs docentes

Conclusión: Este es el primer estudio de estas características realizado en Latinoamérica, por lo que los resultados aquí obtenidos son de gran valor. La evaluación tendía a profundizar en las competencias CM y CP y en menor grado al MCBP. La distribución mostrada en la selección de instrumentos de evaluación por competencia e hito tienden a favorecer los instrumentos de los niveles más bajos de la pirámide (saber y saber cómo). La discordancia existente entre las percepciones de los residentes y los docentes en términos de los instrumentos utilizados y las habilidades cognitivas evaluadas podría interpretarse como una tendencia a la evaluación de habilidades cognitivas de orden inferior y conocimientos biomédicos sobre la adquisición de habilidades psicomotoras. Los resultados muestran desbalance entre lo que se debe aprender (objetivos de aprendizaje), lo que se quiere evaluar (objetivos de evaluación), y como se evalúa (instrumentos de evaluación).

\section{Rol de la Universidad Nacional del Sur en el proceso de acreditación de especialistas en Medicina Familiar de la Federación Argentina de Medicina Familiar y General: Utilización del sistema de evaluación Filomena.}

Badr P (1), Serralunga G (1), Sartori L (2)

(1) Departamento de Ciencias de la Salud, Universidad Nacional del Sur, Argentina (2) Federación Argentina de Medicina

Introducción: La OPS recomienda que las residencias médicas estén orientadas a la resolución de problemas, centradas en la

\section{ARS MEDICA Revista de Ciencias Médicas}


comunidad, posibilitando el aprendizaje en diversos contextos y centrada en competencias de APS. Enfatiza en que las Universidades sean socios activos en la formación de residencias y proponen compartir propuestas novedosas de competencias básicas desarrolladas en diversos países. La complejidad socioambiental del primer nivel de atención demanda evaluaciones de competencias clínicas que reflejen el contexto real de trabajo. En este contexto la Universidad Nacional del Sur colabora formalmente con la Federación Argentina de Medicina Familiar y General, aplicando una herramienta evaluativa que garantiza validez, confiabilidad, pertinencia, y factibilidad, en la instancia de acreditación de especialistas, con énfasis en competencias específicas del médico de familia. El objetivo de este estudio es describir la construcción del sistema de evaluación de competencias "Filomena" desarrollado por la UNS para medicina familiar y su utilidad para el proceso de acreditación de especialistas de la FAMFyG durante 2018.

Métodos: Filomena fue desarrollado empleando el framework open source llamado Cake PHP y utiliza la arquitectura Modelo - Vista - Controlador, que separa las lógicas de diseño (modelo) y presentación (vista). Es una aplicación web centralizada (arquitectura cliente-servidor), accesible desde cualquier navegador web instalado. Se evalúan competencias mediante el diseño de 10 situaciones clínicas que cubran el espectro de problemas en Medicina Familiar. Cada problema tiene especificidad de contenido, y aporta transversalmente a todas las competencias. Las situaciones recrean la dinámica y complejidad real de la consulta ambulatoria, enmarcadas y articuladas en un único contexto socio familiar. Las 15 competencias se agrupan en los 4 momentos de la entrevista orientada al problema:Subjetivo (S), Objetivo(O), Evaluación (E) y Plan de acción (P), y se evalúan mediante 120 conductas observadas en listas de cotejo. El software permite la presentación de información multimedia en forma secuencial, con acceso de todos los usuarios vía web. La validez de constructo se garantizó con técnica Delphi con médicos de familia expertos y la confiabilidad se analizó calculando el Coeficiente alfa de Kronbach con el uso del software SPSS 22

Resultados: Se evaluaron 17 residentes. El desempeño global fue de 54,2 (DE=11,0). La confiabilidad presentó un Coeficiente alfa de Kronbach de 0,84. La aceptabilidad evaluada mediante encuestas resalta la pertinencia y coherencia de la herramienta, su impacto educativo, interfaz amigable, resguardo electrónico, proceso de corrección sistemático y eficiente. Como desventaja se identifican la complejidad de diseño y la limitación operativa que implica la utilización de una PC por evaluado.

Conclusiones: Filomena es una herramienta de evaluación diseñada para currículas basadas en competencias. Su arquitectura de aplicación WEB otorga al sistema robustez, flexibilidad y adaptabilidad a distintos procesos formativos. En Medicina Familiar es válida para resaltar aspectos no biomédicos y logra una representación fiable del contexto profesional del médico de familia, lo cual jerarquiza aspectos del perfil del médico de APS con su correspondiente impacto educativo. Sus características psicométricas, aceptabilidad, impacto educativo y bajo costo la hacen una herramienta útil para su utilización en residencias del primer nivel de atención.

\section{Evolución del Método de Selección de Residentes en Panamá}

Atencio Sary (1), Carrasquilla Sonia (2), Cedeño Marleni (2), Rangel Janett (3)

(1) Facultad de Medicina de la Universidad de Panamá, (2)Caja del Seguro Social de la República de Panamá, (3) Hospital Santo Tomás

Introducción: Abordaremos de manera general el nacimiento de las residencias médicas en los hospitales de la República de Panamá y su vínculo académico con la Facultad de Medicina de la Universidad de Panamá, para luego adentrarnos en los mecanismos utilizados para la selección de los residentes desde su inicio a la fecha; estableciendo las leyes que reglamentan los requisitos para los concursos de residencia y los organismos creados para la supervisión del mismo.

Métodos: Utilizamos el método de investigación histórica (analítico-sintético). Analizamos los sucesos históricos dividiéndolos por periodos, enfocándonos en las especialidades básicas más antiguas para poder conocer sus raíces y partiendo de esta información llevamos a cabo la síntesis que reconstruye y explica "La Evolución de la Selección de Residentes en Panamá".

Manejamos fuentes escritas y orales principalmente. Nos entrevistamos con especialistas egresados de las residencias más antiguas del país, ya que de las mismas no hay muchas fuentes escritas por la manera en que se manejaban a los residentes de aquella época, utilizamos internet y también obtuvimos una valiosa información con documentos como cartas, libros que reposan en la biblioteca de la Facultad de Medicina de la Universidad de Panamá.

Algunos de los hechos históricos más relevantes ligados a las Residencias Medicas en Panamá:

- En 1938, después de finalizar dos años de internado en el Hospital SantoTomas, el Dr. Antonio González Revilla ingresa a la residencia en Cirugía en el recién inaugurado Hospital "Manuel Amador Guerrero" de Colon a través de una entrevista y buenas referencias. 
- En 1947 se crea con el fin de controlar la práctica de las profesiones médicas y afines, el Consejo Técnico de Salud mediante la Ley 66.

- En 1948 Se funda la Academia Panameña de Medicina y Cirugía por el Dr. Antonio González Revilla.

- En 1951 se funda la Facultad de Medicina de la Universidad de Panamá.

- En 1962, después de finalizar dos años de internado en el Hospital Santo Tomas, el Dr. Enero Avilés ingresa a la residencia en Cirugía en el recién inaugurado Complejo Hospitalario Dr. Arnulfo Arias Madrid a través de una entrevista, buenas referencias y aprobación del examen realizado por el Servicio de Cirugía.

- En 1969 se crea el Ministerio de Salud (MINSA), por medio de Decreto de Gabinete №1.

- En 1985 el Hospital Santo Tomás es acreditado como Hospital Docente por el Consejo Técnico de Salud ya tipificado en la Ley N`4 del 10 de abril de 2000.

- En 1988, mediante Resolución No3 se aprueba el Reglamento que regula los requisitos para clasificar a los hospitales de docencia en la Republica de Panamá.

- En 1995 la Facultad de Medicina empieza a otorgar avales académicos a las residencias hospitalarias. Se da comienzo a la figura del residente hospitalario universitario, bajo unos planes y supervisiones más específicas. Las especialidades de Cirugía General, Ortopedia, Medicina Interna, Obstetricia y Ginecología, Pediatría y Psiquiatría, fueron las primeras en organizarse dentro de este nuevo método.

- En 1996 la Facultad de Medicina de la Universidad de Panamá confecciona el Examen de Conocimientos Básicos Generales, con el propósito de ser parte fundamental en los requisitos para optar por una plaza de residencia médica

- En el 2003 la Facultad de Medicina implementa el Examen de Conocimientos Básicos Generales realizado por National Board of Medical Examiners (NBME), el cual reforzó la confianza de los concursantes en el proceso de selección de los residentes.

- En el 2003 por medio del Decreto Ejecutivo №119 se establece el reglamento general de médicos residentes e internos y se contempla la creación de la Comisión Nacional de Docencia Medica.

- En el 2004, por medio de la reunión extraordinaria №3 del Consejo Técnico de Salud, se aprueba el Programa de Internado Medico.

- $\quad$ En el 2004, por medio del Decreto Ejecutivo №222 se crea el Reglamento de los Concursos para las Residencias Medicas.

- En el 2014 la Facultad de Medicina inicia la aplicación del IFOM confeccionado por NBME; como prerrequisito para ingresar al internado medico fortaleciendo así las competencias de los aspirantes al Concurso Nacional de Residencias Medicas.

- En el 2014 el Concurso Nacional de Residencia pasa de ser
Institucional a ser de carácter nacional, siendo la Facultad de Medicina de la Universidad de Panamá la sede de todo el proceso de selección.

- En el 2014 la Comisión Nacional de Docencia de Médicos Residentes e Internos se instala en la Facultad de Medicina, bajo la Resolución Nํ786.

- $\quad$ En el 2018, por medio de la Resolución №2 del Consejo Técnico de Salud, fue aprobada la actualización del Programa Nacional de Internado Médico, el cual es un requisito para participar en el Concurso Nacional de Residencias Medicas.

- $\quad$ En el 2018, por medio del Decreto Ejecutivo №321 se actualiza el Reglamento de Concurso de Residencias Medicas.

- En el 2018 se actualizan los Programas de Residencias Medicas, dándoles carácter de Doctorado y Maestrías Universitarias.

Resultados: Podemos destacar que los resultados de la introducción de nuevos instrumentos en el proceso de selección de los residentes demuestran la transparencia con la que se lleva acabo el concurso, siendo el merito académico el criterio de aprobación de una etapa a la otra.

El IFOM mide el conocimiento central esperado internacionalmente de los estudiantes en puntos críticos a lo largo de su educación médica universitaria. Examina el conocimiento clínico que deben tener los estudiantes o egresados de medicina antes de iniciar el internado medico en Panamá.

Desde que se implemento el IFOM en el 2014, como filtro para entrar en el sistema de salud, se han fortalecido las competencias de los aspirantes al Concurso Nacional de Residencia Medicas.

El Examen de Conocimientos Básico Generales examina en mayor medida el conocimiento en las Ciencias Médicas Clínicas que deben tener los médicos luego de haber culminado el entrenamiento de 2 años en el Internado Medico, el cual equivale a una especialización universitaria en Ciencias Clínicas.

El IFOM y el Examen de Conocimientos Básicos Generales es elaborado por NBME, entidad que reforzó la transparencia en el proceso de selección de residentes.

\section{LICENCIATURA EN MEDICINA}

\begin{tabular}{l|l|l|l}
\hline \multicolumn{4}{c}{ IFOM - NBME } \\
Mayo 2016 \\
\hline \multirow{3}{*}{ Universidad de Panamá } & Inscritos & Aprobados & Porcentaje \\
\cline { 2 - 4 } & 56 & 56 & $100 \%$ \\
\hline
\end{tabular}

*En el 2014 la Facultad de Medicina inicia la aplicación del IFOM elaborado por National Board of Medical Examiners (NBME); como prerrequisito para ingresar al Internado Medico. 


\section{INTERNADO MEDICO}

Examen de Conocimientos Básicos Generales - NBME Febrero 2019

\begin{tabular}{l|l|l|l}
\hline \multirow{2}{*}{ Universidad de Panamá } & Inscritos & Aprobados & Porcentaje \\
\cline { 2 - 4 } & 138 & 129 & $93 \%$ \\
\hline
\end{tabular}

Inscritos en el Examen de Conocimientos Básicos Generales de febrero 2019 que realizaron el IFOM de mayo 2016

\begin{tabular}{l|l|l} 
Inscritos & Aprobados & Porcentaje \\
32 & 32 & $100 \%$ \\
\hline
\end{tabular}

*En el 2003 la Facultad de Medicina implementa el Examen de Conocimientos Básicos Generales elaborado por NBME, como requisito para ingresar a una Residencia Medica, luego de finalizar el internado medico.

Conclusión: La búsqueda del método ideal para la Selección de Residentes en Panamá es lo que la Comisión Nacional de Docencia de Médicos Residentes e Internos está empeñada a encontrar, por medio de la implementación de instrumentos cuantificables apoyados en Decretos y Reglamentos que rigen al Concurso Nacional de Residencia Medica; partiendo del hecho de que no existe la perfección metodológica, sino una adaptación a las realidades actuales.

La Comisión debe velar por la supervisión de los programas de adiestramiento para médicos residentes e internos, al igual que por el cumplimiento del Reglamento Nacional, siendo todos los aspectos y beneficios de obligatorio cumplimiento en todas las instalaciones donde se formen internos $\mathrm{y} / \mathrm{o}$ residentes.

En el esfuerzo de conocer la Evolucion de la Selección de Residentes en Panamá pudimos entender la gran importancia de brindarle la oportunidad a los aspirantes a una plaza de residencia a que demuestren con mertios academicos que son dignos de la misma, lo que da cierta tranquilidad a los futuros pacientes ya que tendran como especialistas a lo mejor de los mejor. Se debe tener claro que el proceso de selección que hay en Panamá no asegura la integridad moral y etica de los ganadores de las plazas, aunque hayan aprobado todas las etapas del Concurso Nacional de Residencia. La Comisión está constantemente trabajando por modernizar los procesos, introduciendo o modificando los instrumentos de evaluación para que sean más objetivos. La Comision pronto contará con un laboratorio de simulación el cual será incorporado al proceso de selección de residentes, con el fin de poder seleccionar los mejores residentes en cada concurso en pro de la salud de la población panameña.

\section{Evaluación de un curso de Seguridad Asistencial para residentes de Medicina Intensiva mediante el modelo de Kirkpatrick}

\author{
Ruiz Carolina (1)(2)(4), Vera Magdalena (1)(3)(4), Navarro Noskar \\ (3), Severino Nicolás (1)(3)
}

(1) Departamento de Medicina Intensiva, Facultad de Medicina, Pontificia Universidad Católica de Chile, (2)Unidad de Paciente Crítico, Complejo Asistencial Dr. Sotero del Rio, (3)Unidad de Paciente Crítico, Hospital Clínico Universidad Católica, (4)Estudiantes del Magister en Educación Médica de la Pontificia Universidad Católica de Chile

Introducción: El ambiente de las Unidades de Cuidados Intensivos (UCls) y la gravedad de los pacientes críticos hace que estos sean especialmente vulnerables a los eventos adversos. Se describen 1.7 errores/paciente por día de UCI y gran parte pueden producir daño significativo o muerte (1). A pesar de esto, los niveles de conocimiento sobre seguridad asistencial (SA) en residentes de distintas especialidades, incluyendo medicina intensiva (MI), son limitados (2). Sin embargo, diferentes estudios han demostrado que el entrenamiento mejora la actitud hacia la SA y reduce los errores (3). Adicionalmente, la SA está considerada de distintas formas en cada uno de los roles y competencias CanMEDS (4). Es por esto, que a partir de 2018 dentro del programa de $\mathrm{MI}$ de la Pontificia Universidad Católica de Chile (PUC) se desarrolló un curso piloto de educación en SA. Este curso se desarrolló en 4 sesiones con metodologías activas de pequeño grupo y tuvo un equipo de docentes multidisciplinario. El objetivo del presente estudio fue evaluar este curso mediante los niveles 1 y 2 del modelo de evaluación de programas educacionales de Kirkpatrick.

Metodología: Se evaluaron los niveles 1 (satisfacción) y 2 A (percepción) del modelo de Kirkpatrick mediante encuestas contestadas por los participantes. Para evaluar la satisfacción se realizó una encuesta tras la 1era y última sesión del curso, y para evaluar la percepción se aplicó una encuesta antes de la 1era sesión y tras finalizar el curso. Ambas encuestas fueron desarrolladas con apoyo de expertos del Centro de Educación Médica PUC. La encuesta de percepción tenía 8 enunciados (relacionados con la relevancia de la SA, el rol de los médicos y el reporte de eventos adversos) a calificar mediante una escala de acuerdo de 5 puntos. La encuesta de satisfacción tenía 11 enunciados (relacionados con la organización del curso, el rol de los facilitatores y el aprendizaje) que se calificaban con una escala de apreciación global de 1 a 7 . Los resultados se describieron me- 
diante porcentajes. Además, se analizó la evolución de las respuestas entre las encuentas iniciales y finales, utilizando el Test de Fisher. Se consideró un valor $\mathrm{p}<0.05$ como estadísticamente significativo.

Resultados: Los 18 residentes del programa hicieron el curso y contestaron las encuestas. En la encuesta de satisfacción inicial sobre $88 \%$ de los enunciados se calificaron bien o muy bien. En la de percepción inicial sobre el $67 \%$ de los enunciados fueron calificados como de acuerdo o muy de acuerdo. Los resultados finales de ambas encuestas mejoraron, llegando al 100\% de satisfacción, sin embargo, no hubo significación estadística en estos cambios.

Conclusiones: Tras realizar este curso piloto de SA para residentes de MI se encontró un $100 \%$ de satisfacción usuaria. Si bien la percepción sobre la importancia de la SA aumentó con el curso, es un nivel del modelo de Kirkpatrick que aún puede mejorar, tanto con la mejoría del presente curso como mediante la introducción de otras actividades sobre educación en SA en el programa de residencia de MI de la PUC.

\section{Referencias:}

Sharp J. To err is human. TCE The Chemical Engineer. 2013. 28 p.

Sandars J, Bax N, Mayer D, Wass V, Vickers R. Educating undergraduate medical students about patient safety: priority areas for curriculum development. Med Teach [Internet]. 2007;29(1):60-1. Available from: http://www.ncbi.nlm.nih.gov/pubmed/17538837

Leonard M, Graham S, Bonacum D. The human factor: The critical importance of effective teamwork and communication in providing safe care. Qual Saf Heal Care. 2004;13(SUPPL. 1):85-90.

Expert M. CanMEDS 2015 OTR Special Addendum. 2016;(December 2016):3-5.

\section{Examen Único Nacional de selección de residentes 2018: Análisis psicométrico y características de los postulantes}

Serralunga Gabriela(1)(4), del Valle Marta(2)(4), Reig María Ernestina(2)(4), Ladenheim Roberta(3), Skoumal Gladys(3), García Dieguez Marcelo(3)(4)

\begin{abstract}
'Departamento Matemática Universidad Nacional del Sur (UNS), Bahía Blanca, Argentina, ${ }^{2}$ Departamento Ciencias de la Salud, (UNS), Bahía Blanca, Argentina, ${ }^{3}$ Dirección Nacional de Capital Humano, Secretaría de Gobierno de Salud, ${ }^{4}$ Centro de Estudios en Educación de Profesionales de la Salud (CEPROS - UNS)
\end{abstract}

Introducción: La Argentina ha progresado desde 2011 en el desarrollo de un examen único nacional para el ingreso a las residencias sumando progresivamente a las diferentes provincias. En el año 2018 el examen ofreció 3123 vacantes y sólo tres distritos quedaron sin participar. El objetivo de este trabajo es estudiar las características de los postulantes y del Examen Único de admisión a residencias para la convocatoria 2018, analizando sus tablas de especificaciones, la calidad de sus preguntas, sus características psicométricas, su capacidad de establecer ranking y el número de preguntas para una confiablidad óptima.

Métodos: Estudio descriptivo. Se utilizaron bases de datos de postulantes y resultados de 2018. Se analizaron: edad, sexo, nacionalidad, universidad de procedencia, puntaje del examen, promedio de carrera y especialidad elegida. Se compararon puntajes entre sexos y nacionalidad mediante prueba T Student. Se determinó el porcentaje de preguntas por área temática; se analizaron las características de las preguntas incluidas utilizando la escala de Galofré, por presencia de viñeta clínica y aplicación de conocimiento. Se comparó el desempeño según área temática mediante ANOVA, y según presencia de viñeta con T Student. Se analizó la confiabilidad mediante la teoría de generalizabilidad, se evaluó confiabilidad del punto de corte y se realizó un estudio $D$ para optimización. Software: SPSS 17 y Edu G para generalizabilidad.

Resultados: El número de postulantes fue 4805; edad promedio $=28,5$ años $(\mathrm{DE}=3,5)$, porcentaje de mujeres $=64 \%$, porcentaje de extranjeros $=20 \%$, promedio en la carrera $=7,0(D E=1,0)$, Instituciones donde cursaron la carrera con mayor número de postulantes: Buenos Aires (22,3\%), Rosario (15,9\%) y La Plata (7,5\%). El 62\% concursa para Instituciones nacionales (Garrahan, Cruce, y Pcia. Bs. As. y municipios asociados) y las especialidades más frecuentes, Pediatría $(15,1 \%)$ y Clínica médica (12,3\%). Puntaje promedio del examen $=63,4(\mathrm{DE}=11,5)$. Se encontraron diferencias estadísticamente significativas entre puntajes de varones y mujeres a favor de estas últimas $(\mathrm{P}<0,001)$ y los postulantes de universidades nacionales obtuvieron en promedio 12,6 puntos más (IC95\% 11,8-13,4) que sus pares de universidades extranjeras.

Áreas temáticas: Salud del adulto y adulto mayor (38\%), Salud del niño y adolescente (29\%), Salud de la mujer (17\%), Salud pública (16\%). Los porcentajes de preguntas con viñeta y de aplicación de conocimientos fueron $65 \%$ y $87 \%$, respectivamente. El $67 \%$ de las preguntas tuvo un valor del Galofré de 3 o más. Dificultad promedio: $68 \%$. No se encontraron diferencias en el desempeño según área temática $(P=0,24)$, ni entre preguntas con o sin viñeta $(P=0,56)$. Resultados del análisis de generalizabilidad: Coeficiente Gabsoluto $=0,84$, Coeficiente Grelativo $=0,87$, confiabilidad del punto de corte phi $(40)=1$. Resultados del estudio D: El Gabsoluto es de 0,81 con 80 preguntas y va aumentando, aunque se ameseta a partir de las 110 preguntas. 
Conclusión: El examen tiene buena confiabilidad, que no mejora considerablemente aumentando el número de preguntas. Su dificultad es adecuada y es temáticamente equilibrado. La mayoría de sus preguntas tienen calidad aceptable con alto porcentaje de aplicación de conocimientos y presencia de viñeta. Se continuará trabajando para la incorporación de los distritos que aún no se sumaron a la propuesta de este Examen único nacional.

\section{Desarrollo de material para familiares de pacientes, potenciando las competencias CanMeds. Experiencia: "Nuestra abuelita Clara está enferma en casa. ¿Y ahora qué?": Integrando a los niños en los Cuidados Paliativos}

Pavlovic Andrés (1), Claro Juan Carlos (2)

(1 Unidad de Geriatría, Departamento de Medicina Interna, (2) Departamento de Medicina Interna, Pontificia Universidad Católica de Chile, Santiago, Chile.

Introducción: El desarrollo de la educación médica por competencias CanMeds está constantemente buscando nuevas herramientas para el desarrollo de las competencias descritas como intrínsecas (líder, colaborador, promotor de la salud, académico) ${ }^{1}$, con el objetivo de una formación integral centrada en el paciente².

Por otro lado, y frecuentemente, el manejo de pacientes en cuidados paliativos implica una sobrecarga física y emocional para su entorno ${ }^{3}$, situación que tiende a producir menor integración de los niños a la experiencia de enfermedad del familiar y su entorno ${ }^{4,5}$. Dados estos antecedentes, un equipo multidisciplinario (staff de medicina interna, diseñadora gráfica, psico-oncólogas infantiles y un residente de geriatría liderando el equipo), desarrolló un libro para ofrecer un recurso a las familias de pacientes en cuidados paliativos, centrado en los niños y sus cuidadores, que eduque respecto al proceso de enfermedad, favorezca la expresión de emociones y permita reconocer recursos para el afrontamiento de esta experiencia.

Métodos: El material desarrollado consiste en una historia de ficción narrada por dos personajes, nietos de una paciente en cuidados paliativos. En el transcurso de la historia el lector (niño/a) tendrá la posibilidad de intervenir el material (dibujando, enumerando, pintando, etc.) a través de actividades que los personajes le invitarán a realizar, además de reflexionar en torno a distintas temáticas propias del proceso de enfermedad (educación sobre la enfermedad oncológica, visibilizar los cambios y adaptarse a ellos, identificar las redes de apoyo, planificar el autocuidado, redefinir la relación/ interacción con el enfermo, expresar emociones y aceptarlas). Además, cuenta con un cuadernillo para el cuidador, donde encuentra sugerencias para facilitar el acompañamiento del niño/a durante este proceso y el abordaje de cada uno de los temas expuestos en el libro con lenguaje y profundidad adecuados para cada edad.

La confección del material se basa en la técnica narrativa, donde por medio de la estimulación de la imaginación, se sugieren cambios y se ofrece una toma gradual de conciencia ante la situación de enfermedad acontecida en el sistema familiar. Para lograr la validez del material, fue construido por un equipo multidisciplinario y la revisión de expertos.

Resultados: El libro se encuentra actualmente publicado y en proceso de divulgación y presentación en congreso. La experiencia de creación de este material informativo para niños con familiares en cuidados paliativos, evidenció cómo el desarrollo de múltiples competencias CanMeds de difícil abordaje y a distintos niveles, como son el rol de comunicador, colaborador, líder y promotor de la salud, es posible a través de la realización de proyectos con el fin de educar e incorporar al paciente y su entorno en su propio cuidado.

Conclusión: Dicho modelo de desarrollo de proyectos, deben ser evaluadas como herramientas válidas para el potenciamiento de competencias CanMeds en residentes en el futuro.

\section{Bibliografía}

McConnell M, Gu A, Arshad A, Mokhtari A, Azzam K. An innovative approach to identifying learning needs for intrinsic CanMEDS roles in continuing professional development. Med Educ Online. 2018;23(1). doi:10.1080/10872981.2018.1497374

Frank JR, Snell L, Sherbino J E. CanMEDs 2015 Physician Competency Framework. CanMEDS 2015 Physician Competency Fram Ottawa $R$ Coll Physicians Surg Canada. 2015:1-30. http://www.royalcollege. $\mathrm{ca} /$ portal/page/portal/rc/canmeds/resources/publications.

LL N. Helping families of patients with cancer. Oncol Nurs Forum. 2005;32(4):743-750 8p. doi:10.1188/05.ONF.743-750

Park EM, Deal AM, Yopp JM, et al. End-of-life experiences of mothers with advanced cancer: Perspectives of widowed fathers. BMJ Support Palliat Care. 2016;6(4):437-444. doi:10.1136/bmjspcare-2015-000976

Almendro E, Strempel P, Andaluza S, et al. La familia en la enfermedad terminal. Control. 2010;(1):1-31 


\section{Evaluación de un programa de inducción para residentes de medicina familiar}

Mora Isabel (1), Rojas, María (1)

(1) Departamento de Medicina Familiar, Escuela de Medicina, Pontificia Universidad Católica de Chile

Introducción: En 2014, la P. Universidad Católica de Chile se comprometió a aumentar su capacidad formativa de especialistas en medicina familiar para contribuir a satisfacer las necesidades planteadas por el Ministerio de Salud. El número de nuevos residentes creció en 2015 en aproximadamente 60\%, lo que ha requerido una reorganización de algunos aspectos formativos de la residencia. Entre otros cambios, se estableció un Programa de Inducción (PI) estructurado e intencionado, con tiempos protegidos y una secuencia de actividades especialmente diseñadas para generar un espacio de orientación y adaptación progresiva de los nuevos residentes durante sus primeras cuatro semanas de especialidad. Las actividades del PI tienen por objetivo introducir a los residentes paulatinamente en las competencias que se espera desarrollen a lo largo de la residencia, así como en aspectos clínicos y administrativos básicos para desenvolverse sin problemas en los espacios académicos y campos clínicos.

El objetivo de este trabajo es Evaluar la satisfacción de los residentes de las cohortes 2015 a 2018 de primer año de Medicina Familiar con el Programa de Inducción.

Métodos: Una vez completado el PI, se les solicita a todos los residentes responder un cuestionario on line (www.e-encuestas.com) de preguntas abiertas y cerradas. El cuestionario está estructurado en 3 partes: [a] valoración general del PI y del material bibliográfico; [b] evaluación de cada actividad realizada; [c] preguntas abiertas solicitando sugerencias y comentarios al PI. Las respuestas son confidenciales.

Resultados: El número de residentes de primer año fue de 19, 17, 19 y 20 para los años 2015, 2016, 2017 y 2018, respectivamente, con un $85 \%$ de sexo femenino.

Dentro de los principales resultados de la sección [a], el 89\% de los residentes se declara "muy satisfecho" con el PI en general. Sobre el $85 \%$ de los residentes destaca que el Programa les da más seguridad para iniciar las actividades de la residencia, y asimismo, les aumenta el interés por la especialidad al profundizar en los principios y prácticas de la disciplina a través de lecturas y talleres de discusión con distintos docentes/facilitadores a quienes consideran expertos en sus respectivas áreas.
Con respecto a la sección [b], las actividades con mejor evaluación fueron el curso Educación Participativa de Adultos, los conversatorios con exresidentes y la recepción/bienvenida de sus pares.

En los comentarios libres (sección [c]), los residentes valoran especialmente la oportunidad de conocerse y generar vínculos entre compañeros, así como conocer los centros de salud donde desarrollarán su formación. Además, lo reconocen como un espacio necesario para involucrarse en su residencia de manera progresiva y ordenada permitiéndoles disminuir la ansiedad inicial de esta nueva etapa.

Conclusión: El aumento del número de residentes en los últimos años ha requerido una reevaluación de varios aspectos de la residencia de medicina familiar. El Programa de Inducción como actividad intencionada y programada ha contribuido a una inserción progresiva de los nuevos residentes gracias a la cual pueden conocer los campos clínicos, los docentes y los aspectos operativos de la residencia, disminuyendo la ansiedad de las primeras semanas.

\section{Selective process of nursing residents by competency assessment: experience report}

Souza Regina (1), Saito Katya (2), Santos Audry (3)

(1) Nursing, Núcleo de Novos Conhecimentos. Sirio Libanes Hospital, (2)Coordinator, Núcleo de Novos Conhecimentos. Sirio Libanes Hospital, (3)Nursing Manager, Núcleo de Novos Conhecimentos. Sirio Libanes Hospital.

Introduction: The Residency modality in nursing is an on-the-job training program considered the best way of qualifying health professionals for professional practice. It was recognized in Brazil by the Ministry of Health and Education and became an important resource to address the problems related to the training of professionals and their performance in the Unified Health System. The selection process for the incursion into the programs is guided by the Ministry of Education, but it is up to the institutions to define their criteria. The Sirio Libanes Hospital had its first program in 2011 and currently has 10 modalities, of which 4 are uniprofessionals and the other 6 nurses train together with other professional categories. Its main objective is to obtain the desired skills in the candidates such as knowledge, communication, teamwork and interpersonal skills. This approach is supported by the literature which shows that attitudes, such as professionalism and decision making, often require more remediation with residents than technical skills. The latter have less influence on the residents' performance. 
Method: The process is carried out in three distinct phases: the written test, the practical test and the interview. The first phase consists of the written test, being eliminatory and in which the cognitive competences are evaluated through multiple choice questions elaborated by an instrumentalized team according to the National Board of Medical Examiners. In the second phase, there is the practical evaluation that is done with clinical simulation of cases, specially constructed for the evaluation of clinical and attitudinal competences. The third and final phase is the interview and curriculum analysis with the program managers referring to the moment in which the competences related to research and communication areevaluated. After these steps, the candidate is classified according to his / her performance throughout the process.

Results: Two hundred and sixty four nurses were selected in the period from 2011 to 2018 , with $80 \%$ of the female population with a mean age of 26 years (SD 4), with little previous professional experience. Fifty-eight percent of the participants were from the southeast region, $14 \%$ from the northeast region, $15 \%$ from the center-west region, $0.7 \%$ from the southern region and $25 \%$ did not report these data.

Conclusion: The aim of this selection model based on competency assessment is to identify the candidates' ability to learn a new task, solve problems and work as a team, reinforcing aspects not evaluated by traditional selection processes that are decisive for the success of candidates and programs.

\section{Elaboración de criterios para el reconocimiento y categorización de nuevas especialidades y su relación con las modalidades de formación a través de la residencia en el Ministerio de Salud y Acción Social de Argentina}

Duré María Isabel (1), Fernández Mariano (1), Cavalieri Mariana (1), Gilligan Clara (1), García Marcelo (1), Barani Mariela (1)

(1) Ministerio de Salud y Desarrollo Social de Argentina

Introducción: Las especialidades médicas son resultado de un proceso de formación específico. En Argentina, la mayor oferta de formación de posgrado en Medicina la constituyen las residencias. El 80\% de ellas son financiadas desde los ministerios de salud (nacional y provinciales). Existen por consenso federal 70 especialidades médicas reconocidas pero cada jurisdicción puede determinar cuáles certificar en su territorio.
Residencias y especialidades se encuentran en íntima vinculación. Con la aparición de nuevas formaciones surge la demanda de reconocimiento de nuevas especialidades y viceversa, dificultando el proceso de planificación de recursos humanos. Se verifica dispersión en los procesos formativos para una misma especialidad: en algunos casos, se promueven residencias básicas, y en otros; pos básicas. Este estudio se propuso explorar el mapa de especialidades médicas en Argentina, sus marcos regulatorios y su correspondencia con los distintos niveles de la residencia.

Método: La investigación se planteó como un estudio descriptivo, utilizando técnicas de recolección primarias y secundarias. Se llevaron a cabo relevamientos sucesivos organizados por núcleos temáticos centrales como: Antecedentes de experiencias de regulación y formación, Historia de la profesionalización médica en Argentina y políticas sanitarias, Estrategias en formación y articulación laboral. Se realizó una encuesta estructurada a los responsables provinciales de recursos humanos en salud. Se analizaron normativas internacionales, nacionales y provinciales identificando número de especialidades médicas, criterios para la inclusión de nuevas especialidades, categorización en especialidades, subespecialidades y otras, relación con la formación y el acceso a la certificación. Se organizaron los resultados sistemáticamente identificando las especialidades comunes y su coincidencia con el proceso de formación. Se analizaron las categorías existentes para la regulación de las especialidades médicas en las provincias y a nivel internacional.

Resultados: Se encontraron un total de 148 especialidades médicas reconocidas en el país y 79 denominaciones de residencias, de las cuales 37 cuentan con financiamiento nacional. Sólo 42 especialidades coinciden en todas las provincias, número similar al de las especialidades comunes del Mercosur y de la Comunidad Europea. Se identificaron y definieron tres categorías dentro de las especialidades: especialidades propiamente dichas o "básicas", especialidades dependientes o subespecialidades que profundizan un área de una especialidad y capacitaciones especializadas comunes a varias especialidades, que se relacionan con nuevos conocimientos, metodologías de abordaje de los problemas o tecnología. El primer grupo se relaciona con las residencias a las que se accede con la formación de grado y los dos restantes con lo que se considera residencias posbásicas o fellows que requieren una residencia previa

Los resultados permitieron elaborar una propuesta con criterios de incorporación de nuevas especialidades y categorización de las vigentes y lineamientos para la elaboración de programas de residencias acorde a dichos criterios.

Conclusiones: La cantidad de especialidades comunes en todas las provincias es acorde a las residencias financiadas a nivel nacional y 
a las que se identifican internacionalmente. EL número de denominaciones de residencias es coincidente con las especialidades del consenso federal. Los procesos regulatorios de reconocimiento de especialidades deben acompañar la planificación de recursos humanos en la materia para una adecuada orientación de la formación a las prioridades de política sanitaria.

\section{Maltrato durante la formación de residentes. Tiempo de terminar con el silenciamiento}

Reboiras Fabiana (1)

(1) Directora Departamento de Educación Instituto Universitario Hospital Italiano, Buenos Aires, Argentina.

Introducción: Las residencias médicas son programas de formación en servicio de dedicación a tiempo completo que implican un alto requerimiento psico-físico para los residentes.

La residencia se caracteriza por una organización fuertemente jerárquico-piramidal y por un alto nivel de interdependencia entre sus miembros.

Existe un elevado número de estudios internacionales que alertan sobre la presencia de prácticas de maltrato al interior de los programas de formación de residentes. Sin embargo son aún escasos los estudios que abordan esta problemática en las residencias latinoamericanas.

Este estudio se propone investigar la presencia de maltrato durante la formación en la residencia médica y su influencia en los procesos de aprendizaje y en el estado físico y psico-emocional de los médicos residentes de clínica médica, pediatría, tocoginecología y cirugía general de dos hospitales de la ciudad de Buenos aires.

Métodos: Se diseñó un cuestionario para ser completado de forma voluntaria, anónima y con el consentimiento informado correspondiente.

Se organizó en cuatro dominios: maltrato educativo, maltrato psico-emocional, acoso sexual y discriminación. Se relevó información demográfica, grado de reporte de las situaciones de maltrato experimentadas, sus principales consecuencias y los responsables de dichas situaciones.

El protocolo de investigación fue aprobado por el Comité de Ética de Protocolos de Investigación del Hospital Italiano de Buenos Aires.

Resultados: Respondieron la encuesta 98/184 residentes de Cirugía General, Clínica Médica, Pediatría y Tocoginecología de 2 hospitales universitarios de Argentina.
El 68\% fueron mujeres. El 98\% experimentó maltrato educativo, el $86 \%$ abuso psico-emocional, el $28 \%$ acoso sexual y el $45 \%$ discriminación.

Las principales acciones de maltrato identificadas fueron: asignación de tareas inapropiadas para el año de formación; falta de reconocimiento al esfuerzo personal; recibir calificaciones verbales degradantes u ofensivas, recibir gritos ante colegas y pacientes.

El 43\% reportó algún tipo de consecuencia atribuible a las situaciones de maltrato vividas. El $33 \%$ de los encuestados señala haber padecido consecuencias físicas tales como Anorexia, Hipertensión, Obesidad, Jaqueca entre otras. El 30 \% reporta consecuencias psicológicas tales como Ataques de pánico, adicción a drogas o alcohol e insomnio entre otras.

El $70 \%$ dice haber sido testigo de situaciones de maltrato a residentes durante su formación.

Los médicos de planta fueron identificados como los mayores responsables en relación al maltrato educativo y psico emocional (45\%), seguidos por los residentes superiores en segundo lugar (36,5 $\%$ ) y el Jefe de residentes en tercer lugar (28,5\%). El 24,5\% identificó al Jefe de servicio como responsable de este tipo de acciones.

El $73 \%$ de residentes que experimentó maltrato no reportó la situación en la institución.

Conclusión: Las prácticas de maltrato están legitimadas y naturalizadas en las residencias estudiadas.La prevalencia de maltrato durante la residencia es alta. El maltrato fue asociado con ser mujer o extranjero.

Hay escasas normativas institucionales al respecto y existe un bajo nivel de reporte de las experiencias de maltrato. La elaboración de protocolos institucionales de prevención y acción podrían proteger a los residentes y animarlos a reportar este tipo de incidentes abusivos.

\section{Relevancia del estudio y destacar su aporte.}

Se espera que este estudio posibilite futuras investigaciones que profundicen acerca de las consecuencias que el maltrato podría producir sobre los procesos de aprendizaje de los residentes y sobre su estado físico y psico-emocional.

A su vez, se podrá también analizar la relación que estas prácticas de maltrato pueden tener con los modelos de atención de los sistemas de salud y la relación médico-paciente y a su vez, su relación con el error en la práctica médica.

Por otra parte, se espera que este estudio sea un estímulo para producir políticas institucionales de prevención de estas prácticas 
durante la residencia, así como mecanismos de reporte y protección hacia los residentes que fueran objeto de maltrato durante su formación.

Finalmente es intención de este trabajo posibilitar debates, que no se encuentran hoy presentes, acerca de la legitimación de ciertas prácticas educativas de extrema exigencia y sacrificio percibidas como necesarias para la formación médica.

\section{¿Qué transformaciones relatan los residentes luego de su rotación en APS con poblaciones en estado de vulnerabilidad en el chaco salteño argentino?}

Facioni Clara (1), Reboiras Fabiana (1), Daels Francisco (2)

(1) Departamento de Educación, (2)Departamento de Alumnos, Instituto Universitario Hospital Italiano de Buenos Aires, Argentina.

Introducción: Una preocupación actual a nivel mundial es el desarrollo de proyectos y programas educativos que formen a los profesionales de la salud en asistencia contextualizada. Los programas de formación con comunidades en situación de vulnerabilidad y sectores rurales han demostrado fortalecer la preparación de profesionales de la salud para futuras prácticas clínicas en contextos diversos. El Hospital Italiano de Buenos Aires en Argentina es un hospital universitario de gestión privada sin fines de lucro. Es un centro de referencia regional y de alto prestigio en la formación de residentes. El Área de Compromiso Social Universitario Ileva adelante el Proyecto Socio-sanitario-educativo en el chaco salteño argentino. Su principal objetivo es comprender el rol social de las intervenciones profesionales en salud y participar como agente de transformación de las problemáticas nacionales. Este estudio ofrece información para evaluar la implementación del Proyecto. Los resultados contribuyen a revisar los programas de formación, las prácticas presentes y ausentes ofrecidas a los residentes en un centro de alta complejidad como el nuestro insertado en el contexto urbano de Buenos Aires.

Métodos: Se trata de un estudio de tipo cualitativo basado en cuestionarios estructurados, entrevistas en profundidad y el análisis de portfolios elaborados por los residentes al finalizar la rotación de un mes de duración. Los cuestionarios se administraron uno antes y otro después de la rotación y son una aproximación al análisis comparativo de: los intereses y expectativas previas y posteriores a la experiencia en terreno, a los aprendizajes esperados y alcanzados, los aportes de la formación previa para la rotación y el impacto de la rotación en el desarrollo profesional futuro.
Resultados: Hasta el momento rotaron 57 estudiantes y profesionales de la salud (residentes, estudiantes avanzados de grado y profesores de las carreras de medicina y enfermería), de los cuales 27 fueron residentes de las siguientes especialidades: clínica médica, pediatría, psiquiatría, psicología,tocoginecología y kinesiología. El $100 \%$ de los residentes relata que la rotación superó sus expectativas. Algunos de los aspectos más valorados fueron el aprendizaje sobre pueblos originarios, la práctica profesional en contextos desfavorables y ser parte de equipos interdisciplinarios. Manifestaron poseer escasa capacitación previa para la atención integral en ambientes vulnerables y con exiguos recursos. Sin embargo, la mayoría concuerda que ha podido aplicar las habilidades clínicas adquiridas durante su residencia.

Conclusión: La rotación brindó la posibilidad de transformar y ampliar los enfoques profesionales, entendiendo el papel social de la práctica. Los resultados nos desafían a revisar la brecha observada entre los programas de las residencias y las competencias requeridas para la rotación.

La experiencia refuerza la importancia de promover este tipo de proyectos comunitarios y rurales que otorgan la oportunidad educativa de desarrollar experiencia profesional en atención primaria de salud en contextos vulnerables a residentes de un centro urbano y alta complejidad como el nuestro.

\section{Evaluación de la Formación Sanitaria del Residentado Médico en la Especialidad de Anestesia, Analgesia y Reanimación en la Facultad de Medicina - UNMSM}

\author{
Tovar Javier (1), Miranda Christian (2)
}

(1) Universidad Nacional Mayor de San Marcos, Facultad de Medicina Humana, Red Prestacional Almenara - Seguro Social de Salud - EsSalud

(2) Red Prestacional Almenara, Seguro Social de Salud - EsSalud, Hospital Nacional Guillermo Almenara - (3)Red Prestacional Rebagliati Seguro Social de Salud - EsSalud, Hospital Nacional Edgardo Rebagliati Martins Departamento de Anestesiología y Centro Quirúrgico.

Introducción: La evaluación del Residente responde a un fin principal, rendir cuentas a la sociedad sobre el resultado de un proceso formativo desarrollado en instituciones sanitarias. La sociedad pone en nuestras manos a licenciados en el área de las ciencias de la salud (medicina, enfermería, etc.), y nos encarga que le devolvamos un especialista competente. Dicha rendición de cuentas tiene dos aspectos básicos, uno legal y otro moral. El legal está, lógicamente, unido al cumplimiento de la legislación correspondiente, a efectos de obtener un título de especialista en 
unas condiciones reguladas a nivel estatal. El moral está unido a nuestro deber con la sociedad y nuestra institución, y al derecho del residente a ser evaluado, a fin de facilitar y mejorar su aprendizaje, garantizando su competencia final. Por tanto, se trata de un sistema de garantías y de responsabilidad social.

Objetivo: Describir la primera la experiencia nacional obtenida en una especialidad médica en la universidad en el Perú en términos de evaluación de Médicos Residentes, así como la correlación de los resultados obtenidos por las herramientas utilizadas entre sí mismas. Considerando que la formación en la especialidad de Anestesiología es la que mayores estándares se debe cumplir.

Métodos: Estudio retrospectivo observacional, en el periodo lectivo 2016. Se realizó la evaluación con una ficha (encuesta) con 49 preguntas las cuales se validaron en un Piloto previamente, el análisis estadístico se realizó por SPSS. principales medidas de resultado validación del instrumento.

Resultados: Nos evidencia los siguientes resultados; En la Acogida e integración en el centro docente en los residentes del primer año entre $33 \%$ aceptable y $29 \%$ satisfactorio existiendo un $40 \%$ entre muy insatisfechos e insuficiente. Estructura de la docencia el 30\% desconoce dicha estructura. Planificación y desarrollo de la formación el $41 \%$ lo considera aceptable, $41 \%$ satisfactorio y no refiere $18 \%$. Seguridad del paciente de su centro. el $26 \%$ lo considera aceptable y otro $49 \%$ satisfactorio. El nivel de competencial que ha alcanzado en el último año de residencia en todos los niveles se evaluados se encuentra por debajo del $25 \%$. Rotaciones; Desarrollo y supervisión del residente en cualquier dispositivo perteneciente a la unidad en todos los niveles se evaluados se encuentra por debajo del $25 \%$ en general y para los residentes de primer año no existe supervisión física en un $87 \%$ y al alta de los pacientes en un $59 \%$. Sesiones clínicas y bibliográficas: Comunicación y desarrollo; Secciones Clínicas lo considera aceptable 43\% y otro satisfactorio 23\%. Actividades de investigación: Comunicación y desarrollo; Participa en actividades o proyectos de investigación Muy insuficiente $30 \%$ e insuficiente $64 \%$. Evaluación anual; No Conoce los criterios que se aplican para realizar las evaluaciones anuales y finales el 64\%. Quejas y reclamaciones de los residentes; El 79\% No conoce el mecanismo de tramitación de quejas de los residentes al proceso de docencia. Comunicación de resultados; resultados de la encuesta anual de satisfacción de residentes de su hospital /UPG. El 81\% no le comunican y desconocen si se realizan. La Valoración General de la especialidad; En 4\% es muy insuficiente, $13 \%$ insuficiente, $35 \%$ aceptable, $37 \%$ satisfactorio, muy satisfactorio $6 \%$, no refiere $5 \%$.

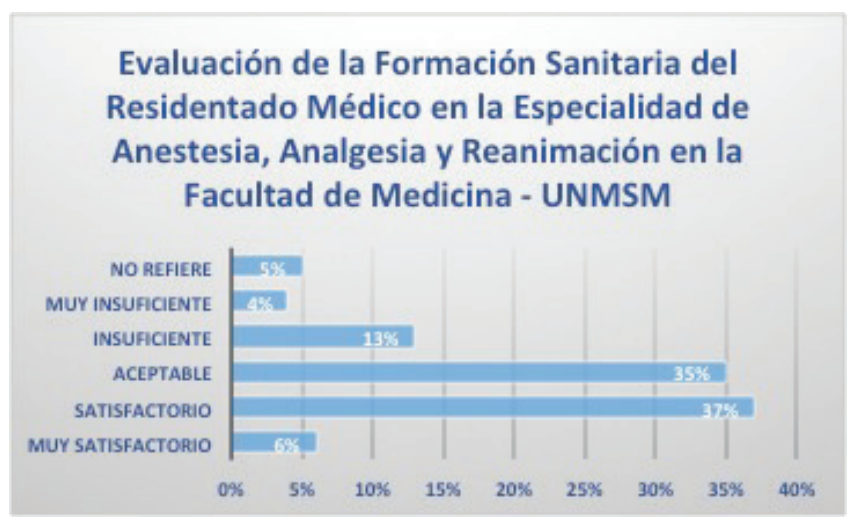

Podemos afirmar que amerita proponer y validar un nuevo formulario de evaluación formativa basada en siete dominios extendiendo, un formulario para las entrevistas tutor- residente, feedback que incluye una autoevaluación de competencias y proponer un portafolio (memoria) reflexivo por competencias. El tamaño de la muestra es de 100 Médicos Residentes de un total de 150. (año 2016).

Conclusión: Es el primer estudio realizado a una Especialidad Médica en la formación en Perú, se puede afirmar que si bien tiene la Facultad e de Medicina Humana - Universidad Nacional Mayor de San Marcos, en una de pocas por no decir la única que tiene todos sus programas estructurados en la formación de Médicos Residentes. Observamos al evaluar a una de las especialidades emblemáticas en la atención de pacientes y con mayores exigencias en la formación, se determinó que existen deficiencias que deben se consideradas y reformular para su intervención, así como las que no tiene la misma exigencia de esta especialidad en la formación en la Universidad como en las otras universidades en la formación de Médicos Residentes.

\section{Implementación de un curso de habilidades en comunicación para residentes: experiencia de 3 años}

Beltrán Daniela (1)(2), Núñez Carolina(3), Vergara Claudia(1), Claro Juan (1)(2), Monsalve Ximena (1)(2), Sepúlveda Paola(1)(2)

\section{(1) Medicina Interna Facultad de Medicina, Pontificia Universidad Católica de Chile \\ (2) Médico Académico, Pontificia Universidad Católica de Chile \\ (3) Residente Medicina Interna Facultad de Medicina, Pontificia Universidad Católica de Chile}

Introducción: Las habilidades comunicacionales (HC) son una de las competencias esenciales de un médico y su enseñanza 
forma parte del core curriculum de las escuelas de medicina y de nuestro marco de referencia CanMEDS. Las HC pueden y deben ser enseñadas, entrenadas y evaluadas. Deben ser comprendidas como una necesidad multidimensional, enfocada en establecer una relación médico-paciente-familia con atención centrada en el paciente, en las relaciones con el equipo de salud, obtención de información médica relevante y el rol del médico como educador. Sin embargo, los médicos no entrenamos estas habilidades, en parte, por sobrevalorar la propia experiencia como entrenamiento suficiente. Desde el año 2015 se imparte un curso obligatorio para residentes de 1er año de Medicina Interna y desde el año 2017 como curso transversal electivo para residentes de otras especialidades en nuestra Escuela de Medicina. El objetivo del presente trabajo es describir las características de los residentes que han participado y los resultados de 6 cohortes.

Métodos: Se realiza un análisis descriptivo de los antecedentes de los residentes que han realizado el curso respecto a variables: demográficas, experiencia laboral previa, especialidad y calificación final del curso. Los instrumentos de evaluación del aprendizaje utilizados de forma sumativa son pruebas escritas, ensayos con pauta y escenarios con paciente simulado con pauta de apreciación. Se realiza un análisis estadístico de aquellas variables con IC al 95\%.

Resultados: El curso ha sido realizado por 112 residentes en sus 6 versiones. El 49\% (55) son residentes mujeres y el 51\% (57) hombres. Presentan una edad promedio al inicio del curso de 27 años (24 - 34) y un 54\% (60) presenta experiencia laboral previa de más de 3 meses. El 64\% (72 residentes) pertenece a la especialidad de medicina interna y un $36 \%$ a otras especialidades que se subdividen en: Geriatría 20\% (10), Diabetes y Nutrición 16\% (8), Medicina Familiar 14\% (7), Psiquiatría del adulto e infantil 10\% (5), Ginecología y Obstetricia 8\% (4) y otras $12 \%(6)$ (resultados se resumen en tabla $n^{\circ} 1$ ). La calificación promedio obtenida es de $6,3(5,1-7,0)$ con una reprobación del $3 \%$ por desempeño insuficiente en el escenario de paciente simulado. No hay diferencias estadísticamente significativas al comparar promedios de calificaciones finales del curso por variables de género (hombres 6,30 vs mujeres 6,21), edad al inicio del curso (menor 30 años 6,29 vs igual o mayor 30 años 6,10 ) y experiencia laboral previa (sin experiencia 6,32 vs con experiencia 6,20 ). Por la baja cantidad de residentes en la rama de especialidades quirúrgicas, no es posible realizar una comparación entre el aprendizaje obtenido entre especialidades médicas versus quirúrgicas.
Tabla n ${ }^{\circ}$ : Características demográficas participantes curso de comunicación

\begin{tabular}{l|l|l}
\hline Sexo & Femenino & Masculino \\
\hline & $49 \%(55)$ & $51 \%(57)$ \\
\hline Edad promedio & 27 años & \\
\hline Experiencia laboral previa & SI & NO \\
\hline & $54 \%(60)$ & $46 \%(52)$ \\
\hline Especialidad de origen & $\begin{array}{l}\text { MEDICINA } \\
\text { INTERNA }\end{array}$ & OTRAS \\
\hline & $64 \%(72)$ & $36 \%(30)$ \\
\hline
\end{tabular}

Conclusión: Las HC pueden ser enseñadas independiente de su etapa profesional y un curso de estas características demostró ser factible y sostenible en el tiempo. Factores como la edad, sexo o experiencia laboral previa no afectan significativamente el rendimiento de los alumnos.

A medida que aumenten los participantes provenientes de otras especialidades, se podrá hacer un análisis que determine si hay diferencias y necesidades de cambios en las metodologías utilizadas para subgrupos de residentes.

\section{Proyecto de Evaluación al Docente de Especialidades Médicas}

\author{
Aguirre Carlos (1), Grez Marcela (1), Garrido Verónica (2)
}

(1) Dirección de Postgrado, Escuela de Medicina, Pontificia Universidad Católica de Chile. Santiago, Chile, (2)Centro de Educación Médica y Ciencias de la Salud, Escuela de Medicina, Pontificia Universidad Católica de Chile. Santiago, Chile.

Introducción: Los programas de especialidades médicas están en permanente búsqueda de mejora de sus metodologías y procesos de evaluación, con el fin de asegurar la calidad de la docencia y el aprendizaje de la educación médica.

Uno de los insumos necesarios para la mejora continua de los Programas de Especialidades Médicas es la evaluación de sus docentes, permitiendo una retroalimentación efectiva que aporte a la mejora del desempeño docente y del Programa de Especialidad. De esta forma, este proyecto busca poner en práctica la evaluación a la docencia tutorial por parte de los residentes en distintos contextos, incluyendo contextos hospitalarios, ambulatorios y quirúrgicos a través del uso de tecnologías de la información que permitan elaborar una base de datos confiable y objetiva. 
Métodos: Para la evaluación docente en los programas de especialidades de la Escuela de Medicina UC de la se proponen 3 etapas de Implementación del Proyecto de Evaluación a la Docencia:

\section{1) Primera Etapa}

\section{Planificación del Proyecto y Elaboración de Encuesta}

Se define:

- Utilización de Pauta de Evaluación MEDUC-PG141

- Información de Rotaciones y Docentes asociados a éstas

- Envío de encuesta online trimestralmente vía email

- Centralización de datos en Excel General con todos los docentes evaluados, con emisión anual de Informe de Resultados a cada Jefe de Programa.

\section{2) Segunda Etapa}

\section{$\underline{\text { Recolección de Datos }}$}

- Presentación del instrumento en reuniones de residentes, aclaración de dudas y entrega de información sobre pasos a seguir.

- Período de Evaluación: Envío de link vía e-mail, respuesta de consultas y recordatorios. Una vez finalizado el período, se envía al Jefe de Programa el recuento de encuestas contestadas, cantidad de docentes evaluados y promedio general parcial de cada pregunta para análisis global de fortalezas y aspectos a mejorar.

\section{3) Tercera Etapa}

\section{Entrega de resultados y retroalimentación.}

Etapa aún no desarrollada porque aún no se cumple un año de implementación ni se alcanza el $\mathrm{N}^{\circ}$ mínimo de encuestas por docente para elaborar un informe objetivo.

Resultados: Inicio del Proyecto y recolección de datos con Programas de Especialidades 2018 - 2019

\begin{tabular}{l|l|l}
\hline Programa de Especialidad & $\mathbf{N}^{\circ}$ de Residentes & N Encuestas \\
\hline Pediatría & 63 & 309 \\
\hline Cirugía General & 33 & 211 \\
\hline Medicina de Urgencia & 30 & 26 \\
\hline Traumatología & 23 & 45 \\
\hline Anatomía Patológica & 12 & 111 \\
\hline Oncología & 4 & 12 \\
\hline Oftalmología & 13 & 34 \\
\hline Ginecología y Obstetricia & 26 & 29 \\
\hline Medicina Familiar Adulto & 33 & 45 \\
\hline Medicina Familiar Niño & 26 & 60 \\
\hline Psiquiatría Adultos & 20 & 70 \\
\hline Medicina Interna & 67 & 750 \\
\hline
\end{tabular}

ARS MEDICA Revista de Ciencias Médicas
Conclusión: El proyecto se encuentra actualmente en una segunda fase de recolección de datos, extendiendo el proyecto de evaluación de docentes a la mayor cantidad de programas de especialidad, con el fin aumentar el número de respuestas por docente.

Se ha evaluado positivamente la presentación del instrumento a Docentes y Residentes en reuniones o jornadas de estudio, aclarando dudas al mismo tiempo de generar fidelización e incrementar la participación de los residentes.

Dentro de los próximos desafíos se encuentra el generar mecanismos de incentivo a la participación de los residentes; definir un número de encuestas para considerar válidos los resultados expuestos; lograr una adecuada retroalimentación a los docentes desde los resultados y conclusiones que arroje la encuesta.

\section{Referencias:}

Pizarro, Margarita et al. Evaluación de docentes clínicos de Postgrado: desarrollo y propiedades psicométricas del instrumento MEDUC-PG14. Rev. méd. Chile. 2015, vol.143, n.8, pp.1005-1014. ISSN 0034-9887.

\section{Enseñando habilidades comunicacionales: satisfacción de los residentes a 3 años de implementación de un curso}

Nuñez Carolina (1), Vergara Claudia (1), Claro Juan (1), Sepúlveda Paola(1), Beltrán Daniela (1)

(1) Departamento de Medicina Interna, Pontificia Universidad Católica de Chile

Introducción: Las habilidades comunicacionales (HC) son una de las competencias esenciales para ser un buen médico y forman parte de los roles del Modelo de Competencia CanMEDS que ha sido adoptado por nuestra Universidad. Desde el año 2015 se implementa un curso de HC para residentes de primer año de Medicina Interna, que en el año 2017 se incorpora como curso transversal para residentes de otras especialidades. La modalidad es mixta, con b-learning y sesiones de carácter teórico práctico, incorporando en sus metodologías vodcast, ensayos, feedback y role play. Presentamos los resultados de evaluación del nivel 1 de Kirkpatrick (reacción) recopilados desde el año 2017, con el objetivo de evaluar el grado de satisfacción de los residentes con respecto a dicho curso.

Métodos: Se confecciona un cuestionario de 18 preguntas en escala de Likert de 4 niveles y una pregunta de respuesta no estructurada. Este instrumento evalúa aspectos que se categorizan 
en contenidos (4 preguntas), metodologías (8), aplicabilidad (5) y grado de satisfacción global (1). Se aplica cuestionario de forma presencial de forma inmediata al finalizar el curso. Se analizan los resultados agrupando los ítems en favorables ("de acuerdo" y "muy de acuerdo") y en desfavorables ("en desacuerdo y "muy en desacuerdo"). Se clasifican los comentarios según aquellos con contenido positivo o aspectos a mejorar.

Resultados: Se aplica el cuestionario a 43 estudiantes, de los cuales se obtiene 41 cuestionarios respondidos de forma completa ( $95 \%$ tasa de respuesta). El $42 \%$ de los residentes son de Medicina Interna. El $88 \%$ de los participantes recomendaría el curso a un colega. Los resultados según categorías: un 95\% considera que lo aprendido es aplicable a la práctica clínica, el 92\% está satisfecho con el contenido y un $88 \%$ con la metodología implementada - destacando el uso de role play como una metodología aceptada por los residentes-. De los aspectos con menor grado de aceptación (76\%) fueron los ensayos semanales. Respecto al análisis de la pregunta de respuesta no estructurada, se obtuvieron 9 comentarios positivos y 17 comentarios como aspectos a mejorar, en su mayoría enfocado en cómo mejorar la experiencia respecto al uso del ensayo como metodología de evaluación para el aprendizaje.

Conclusiones La implementación de un curso de HC es factible en los programas de residencia y es bien aceptado por los residentes. Para su éxito es necesario que el aprendizaje sea situado para mantener la percepción favorable de los residentes de su aplicabilidad. Debemos trabajar en cómo mejorar la experiencia de las actividades reflexivas y su importancia en la adquisición de dominios actitudinales.

\section{Habilidades comunicacionales: enseñando a los docentes}

Sepúlveda Paola (1), Claro Juan(1), Vergara Claudia (1), Núñez Carolina(1), Beltrán Daniela (1)

(1) Departamento de Medicina Interna, Pontificia Universidad Católica de Chile.

Introducción: Las habilidades comunicacionales $(\mathrm{HC})$ son una de las competencias esenciales de un médico y deben ser enseñadas formalmente tanto en pre como en posgrado. En los últimos años, nuestra institución está en el proceso de adoptar el modelo de competencias CanMEDS, en el cual el rol comunicador es uno de los 7 dominios centrales que deben ser enseñados y evaluados en un programa de postgrado. En el año 2011 se aplicó una encuesta en estudiantes de medicina, residentes y docentes para evaluar la percepción acerca de su preparación para enfrentar desafíos comunicacionales en nuestra institución. En dicha encuesta, se descubrió una necesidad curricular, ya que sólo la mitad de los encuestados habían recibido entrenamiento formal en $\mathrm{HC}$ y este porcentaje fue constante si se separaba por estamento. En este contexto, desde el año 2015 se implementó un curso obligatorio para los residentes de $1^{\text {er }}$ año de Medicina Interna. Posteriormente, desde el año 2017 se extendió a otros programas de postgrado como curso electivo. Dado el éxito de la implementación a nivel de residentes, se realizó una evaluación de necesidades de los docentes de Medicina Interna y se diseñó un curso piloto, teórico práctico de HC para docentes, incorporando role play con paciente simulado. El objetivo de este trabajo es mostrar los resultados de dicha encuesta y evaluar el resultado de un curso piloto para docentes.

Métodos: Se confeccionó un cuestionario de 30 preguntas con escala de Likert de 4 niveles, para evaluación de necesidades y se aplicó online a todos los docentes del Departamento de Medicina Interna, previo al inicio del curso. En base a los resultados de esta encuesta, se diseño un curso de modalidad presencial, en formato de pequeño grupo (8 cupos), de 16 hrs cronológicas, que se realizó en un periodo de 4 semanas. Se confeccionó una pauta de observación de $\mathrm{HC}$ en un escenario con paciente simulado, la que fue aplicada al inicio y luego al finalizar el curso. Se realiza un análisis descriptivo del cuestionario inicial y un análisis estadístico con $\mathrm{t}$ de Student para la pauta de observación pre-y post-curso piloto.

Resultados: Respecto a la encuesta, se obtuvo una tasa de respuesta de $52 \%$ por parte de los docentes. Un $88,6 \%$ de los encuestados refirieron sentirse preparados para enfrentar los distintos ámbitos de las HC abordados. Sin embargo, un 72\% negó entrenamiento formal para la adquisición de esta competencia. Respecto al curso piloto, se implementó durante enero del 2018, en el cual participaron 6 docentes de Medicina Interna de manera voluntaria. En la evaluación diagnóstica en escenario de paciente simulado, según pauta de observación, se obtiene una media del porcentaje de logro del $63 \%$ versus $80 \%$ al finalizar el curso, siendo una diferencia estadísticamente significativa ( $p=0.046)$.

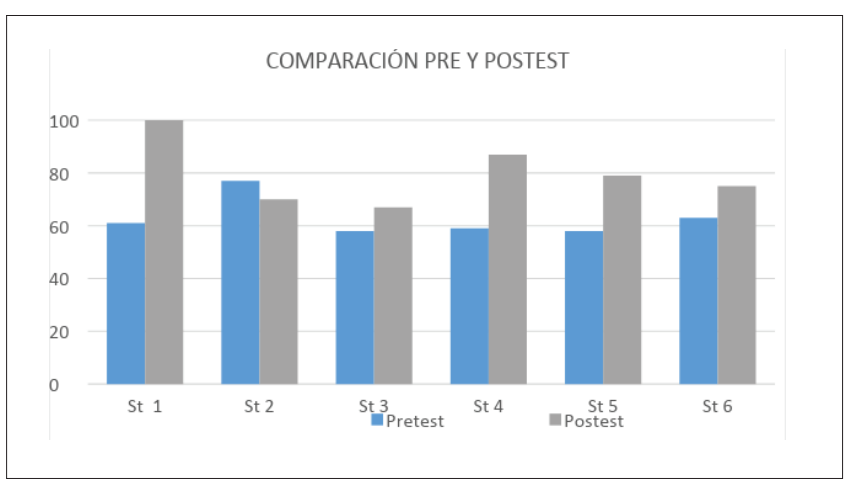

\section{ARS MEDICA Revista de Ciencias Médicas}




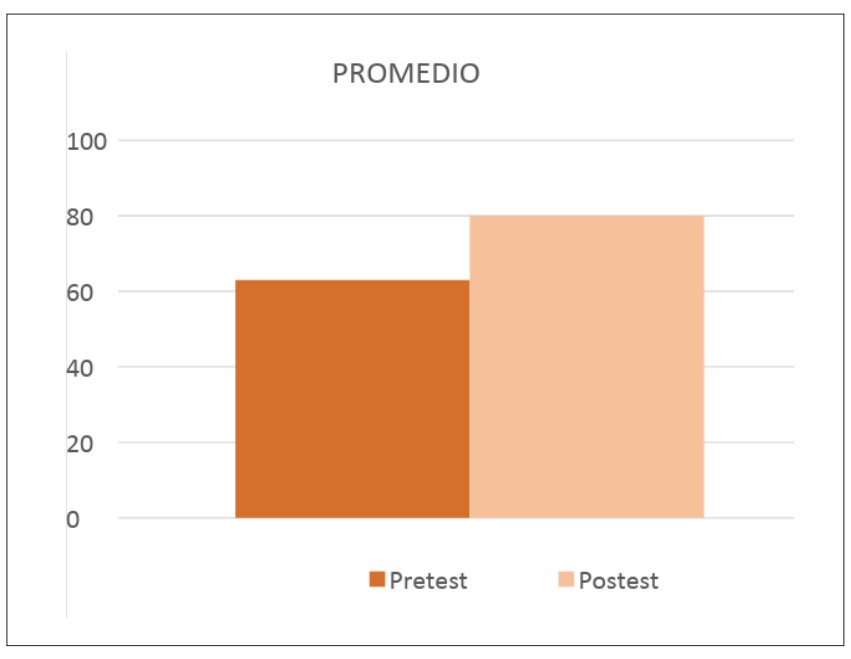

Conclusión: Si bien la encuesta muestra una percepción de los docentes de que tienen la preparación adecuada y necesaria para enfrentar desafíos comunicacionales, la implementación de un curso formal de esta competencia, demostró ser útil en mejorar las destrezas en HC. Se hace necesario promover la enseñanza formal en $\mathrm{HC}$ e incorporarla como un área de desarrollo docente, ya que demuestra ser necesaria y poco reconocida como aspecto a mejorar. Esperamos que este sea el inicio de un curriculum formal de desarrollo académico en nuestra Institución y a futuro, a nivel de todo el país.

\section{Conductas Disruptivas en Ambiente de Pabellón: Un Estudio Cualitativo en Equipos Multidisciplinarios de Salud}

\author{
Campos Mauricio (1), Zúñiga Denisse (2), Lira María (1), Sepúlveda \\ Macarena (1), Calderón Maribel (1), Mery Pamela (1)
}

(1)Departamento de Ortopedia, Facultad de Medicina, Pontificia Universidad Católica de Chile. Santiago, Chile, (2)Centro de Educación Médica y Ciencias de la Salud, Facultad de Medicina, Pontificia Universidad Católica de Chile. Santiago, Chile, (3)Facultad de Psicología, Universidad Alberto Hurtado. Santiago, Chile.

Introducción: El estudio de las conductas disruptivas en ambiente de pabellón ha sido tema de alto interés en los últimos años. Dos hallazgos resultan especialmente preocupantes: los cirujanos son los profesionales que presentan mayores tasas de comportamiento disruptivo al interior del equipo de salud, y se establecen relaciones entre conductas disruptivas, el ambiente laboral y la seguridad del paciente. No se encontraron estudios que investiguen el fenómeno de conductas disruptivas desde la perspectiva de multidisciplinaria. El objetivo del estudio fue explorar las percepciones de los diferentes integrantes del equipo de salud respecto la dinámica del trabajo en pabellón y la presencia de conductas disruptivas en este ambiente.

Métodos: Estudio cualitativo de carácter exploratorio enmarcado en una lógica analítica- relacional. Se realizaron 20 entrevistas individuales semiestructuradas a cirujanos, anestesistas, residentes y personal del equipo de salud en contexto de pabellón de una institución. Dos investigadoras entrenadas del equipo realizaron las entrevistas mediante un guion temático, hasta lograr saturación de los datos. Se realizó un análisis de las entrevistas en los niveles de codificación abierta y axial del enfoque de teoría fundada. Para la validación de los resultados se utilizó la triangulación entre investigadores. Los análisis se realizaron con el software Atlas.ti v8.0.

Resultados: En el contexto quirúrgico existen condiciones que disponen el actuar de los agentes involucrados en el trabajo de pabellón, quienes reaccionan -o no- frente a las dificultades con comportamientos disruptivos hacia el equipo de trabajo. Los principales problemas identificados se asocian a la infraestructura, relaciones interpersonales y falencias organizacionales.

Existen factores personales y culturales que condicionan la respuesta de los involucrados, y que pueden conducirlos a presentar conductas disruptivas en pabellón. Los factores identificados fueron las normalizaciones de la disrupción, relaciones jerárquicas dentro del equipo de trabajo, diferencias de género, generacionales y socioculturales.

Se identificaron relaciones potencialmente disruptivas entre los distintos estamentos que conforman el equipo de pabellón. Dentro de estas relaciones, el estamento cirujano mostró ser el estamento con mayor cantidad de denuncias de conductas disruptivas.

Las principales conductas disruptivas identificadas se clasificaron en aquellas verbales correspondientes a gritos, groserías e insultos y no verbales como tirar cosas, agresividad y aquellas relacionadas a la falta de profesionalismo (mascar chicle, mirar el celular). No se identificaron estrategias institucionales para enfrentar comportamientos disruptivos en el ambiente de pabellón. A nivel individual existen diferentes estrategias, las cuáles van desde la normalización hasta el rechazo de dichas conductas. Se identificaron como consecuencias de conductas disruptivas la alteración del ambiente en pabellón y seguridad del paciente.

Conclusión: El ambiente en pabellón presenta características particulares que ponen en tensión las dinámicas relacionales de trabajo que pueden influir en los resultados clínicos, y en la percepción del ambiente educacional y laboral de todos los integrantes del equipo de salud. En el contexto de un hospital docente, sería relevante poder comprender con mayor profundidad cuál 
es la percepción que tienen los residentes sobre las conductas disruptivas en ambiente de formación en pabellón e identificar los aspectos a mejorar.

\section{Calidad de vida en residentes de programas de especialidades médicas de una Universidad Chilena.}

Irribarra Luis (1), Lira María (1), Mery Pamela, Irarrázaval Sebastian (1), Anwandter Philipp (1), Klaber laniv (1), Campos Mauricio (1)

(1)Departamento de Ortopedia, Facultad de Medicina, Pontificia Universidad Católica de Chile. Santiago, Chile

Introducción: La calidad de vida (CV) es un tema relevante en la literatura médica. Los programas de residencia de especialidades médicas presentan una gran exigencia académica y emocional, teniendo un impacto negativo en la salud mental. El objetivo de este estudio fue evaluar la calidad de vida en residentes de programas en especialidades médicas.

Métodos: Estudio transversal. Se invitó a participar a todos los residentes de especialidades médicas de la Pontificia Universidad Católica de Chile. Se utilizó una encuesta online, se recolectaron datos sociodemográficos y se evaluó la calidad de vida a través del cuestionario WHOQOL-BREF (puntaje de 0 a 100), validado al español. Se evaluó el puntaje global obtenido en los dominios de la encuesta: Salud física, Salud psicológica, Relaciones interpersonales y Entorno. Se compararon los puntajes de cada dominio según: tipo de especialidad (quirúrgica vs médicas), sexo, estado civil, actividad física y consumo de tabaquismo, metilfenidato, modafinilo y marihuana. Se utilizó la prueba de chi cuadrado para variables categóricas, T-student y ANOVA para medias. Se realizó regresión lineal multivariante paso a paso utilizando las mismas variables analizadas de forma independiente, reportando el efecto de cada variable sobre los puntajes en cada dominio de calidad de vida. Se utilizó el software estadístico STATA v14.0.

Resultados: Los puntajes globales fueron: Salud física (62.45), Psicológica (61.60), Relaciones interpersonales (57.48) y Entorno (62.9). En el análisis multivariado hubo, en el dominio de Salud física, una asociación indirecta con la variable consumo de metilfenidato (-8.14 puntos) y una relación directa con los residentes que practican actividad física (+ 5.6 puntos). En Salud psicológica, estudiantes de segundo y tercer año y con consumo de metilfenidato presentaron una relación indirecta $(-5.06,-6.14$ y -7.24 puntos, respectivamente), mientras que tener pareja y ser hombre se asociaron de forma directa a mejores puntajes (+ $5.13 \mathrm{y}+5.20$ puntos, respectivamente). En
Relaciones Interpersonales, los menores puntajes correspondieron a aquellos residentes que tienen hijos (-9.9 puntos), mientras aquellos que realizan actividad física y tienen pareja presentaron mejores puntajes ( $+5.67 \mathrm{y}+10.94$ puntos, respectivamente). Estas dos últimas se variables asociaron también a mejores puntajes en Entorno ( $+5.40 y+6.24$ puntos), mientras que ser extranjero, tener hijos y ser de segundo año fueron predictores de peores puntajes (-7.07; -6.6 y -4.9 puntos, respectivamente).

Conclusión: Se observó que la CV se modifica según las variables estudiadas. Dentro de las variables modificables que afectan la calidad de vida se encuentran el consumo de metilfenidato y la actividad física. Respecto a esta última, influyó en los puntajes de tres de los cuatro dominios.

\section{Implementación de una rotación de Lactancia Materna como modelo de enseñanza del rol de Promotor en Salud (CANMEDS)}

Karzulovic Lorena (1), Escalante María (2), Valdebenito Carolina (3)

(1) Departamento de Pediatría, División de Pediatría, (2)Departamento de Neonatología, División de Pediatría, (3)Departamento de Pediatría, División de Pediatría, Pontificia Universidad Católica de Chile, Santiago, Chile.

Introducción: El programa de especialización en Pediatría de la Pontificia Universidad Católica de Chile adscribe al modelo de formación por competencias CanMEDS. Una de estas competencias es el rol del médico como "Promotor en Salud", que busca contribuir a la mejora de la salud, involucrando su prevención, promoción y protección.

La lactancia materna representa el ideal de nutrición por sus múltiples beneficios para los lactantes, sus madres, sus familias y la sociedad. Por esto, las intervenciones para promover la lactancia materna son de las más costo-efectivas en salud.

La evidencia demuestra que las intervenciones que apoyan la lactancia materna realizadas por profesionales de la salud son efectivas para aumentar la proporción de madres que amamantan, por lo cual el pediatra debe tener una formación que le permita reconocer condiciones que ponen en riesgo el inicio, establecimiento y mantención de una lactancia exitosa como oportunidades de intervención.

Es un rol fundamental del pediatra promover la lactancia materna en diferentes situaciones, ya sea en el apoyo del establecimiento de la lactancia materna con madres puérperas o en la supervisión de 
salud. También debe protegerla ante amenazas para su mantención, como son las hospitalizaciones neonatales o de lactantes. Es por esto que consideramos que para lograr el aprendizaje de este rol era necesaria una rotación que permitiera abordar la lactancia como tema central, llevada a diferentes situaciones habituales de la práctica pediátrica.

Se presenta la experiencia de la implementación de una Rotación de Lactancia Materna para becados de pediatría, como un modelo para enseñar el rol de promotor en salud.

Métodos: El programa fue diseñado por docentes de pediatría de la Pontificia Universidad Católica de Pediatría. El propósito de la rotación es desarrollar conocimientos, habilidades y actitudes necesarias para promover y proteger la lactancia materna en la práctica pediátrica, independiente del lugar donde se ejerza. Esto incluye objetivos específicos de promoción de lactancia materna en condiciones fisiológicas y promoción y protección de la misma en situaciones especiales.

Se diseñó una rotación de 4 semanas de actividades docentes clínicas tanto en el escenario ambulatorio como hospitalizado (pediatría ambulatoria, neonatología, pediatría hospitalaria) y con diferentes profesionales de la salud (pediatras, fonoaudiólogos, enfermeras, matronas).

Se realizó una encuesta de satisfacción a la residente que realizó el piloto de rotación.

Resultados: La evaluación de la rotación por la residente fue positiva en cuanto a los contenidos, metodología docente y valoración de lo aprendido para su práctica clínica. Se evalúa positivamente que el enfoque sea multicéntrico y multidisciplinario. Como valoración global, destacó especialmente el haber logrado incorporar el concepto de que la lactancia se ve amenazada muchas veces y el rol del pediatra debe ser activo en su protección y anticiparse a estos momentos (prematurez, hospitalización, retorno al trabajo, etc). También refiere que desde que realizó la rotación, trabaja más en conjunto con otros profesionales de la salud. Como aspectos a mejorar sugiere entregar un mayor contenido teórico al inicio de la rotación para que el resto de las actividades sea más productivo.

Conclusión: La rotación de lactancia materna ofrece una oportunidad única para desarrollar el rol de promotor en salud en los residentes de pediatría. La promoción de la lactancia materna beneficia a la sociedad y los pediatras deben promoverla y protegerla activamente. Esta primera versión fue evaluada positivamente y permitió lograr su objetivo docente. Se evaluará incorporar más contenido teórico en próximas versiones.

\section{Implementación de un Curso de Medicina Basada en Evidencias modalidad b-learning para residentes como modelo de enseñanza del Rol Académico (CANMEDS)}

\author{
Karzulovic Lorena (1), Lara Bárbara(2), Cifuentes Lorena(3), \\ Letelier Luz María (4)
}

(1) Departamento de Pediatría, División de Pediatría, (2)Sección de Medicina de Urgencia, (3)Departamento de Pediatría, Centro de Evidencia UC, (4) Departamento de Medicina Interna, Pontificia Universidad Católica de Chile, Santiago, Chile

Introducción: Una competencia clave del rol "Académico" del médico es integrar a la práctica la mejor evidencia disponible. Para lograr esto, es indispensable contar con los conocimientos y habilidades necesarias para evaluar críticamente la confiabilidad y la aplicabilidad de la literatura relacionada con salud.

Tradicionalmente, algunos programas de especialidad imparten cursos de medicina basada en la evidencia (MBE) en horario protegido para los residentes del programa con metodología presencial. Estos cursos, aunque abiertos a otros programas, no logran abarcar a todos los residentes de todos los programas de postgrado. Una encuesta de satisfacción realizada a residentes que habían realizado este curso reveló que a pesar de que la gran mayoría valoraba la utilidad de lo aprendido para su práctica profesional, un $61,6 \%$ habría preferido una modalidad online o blended.

La evidencia sugiere que la metodología B-learning no es inferior a la metodología presencial convencional y podría tener ventajas para los estudiantes como la autogestión del tiempo o poder acceder desde lugares geográficos alejados.

Se presenta la experiencia de la implementación de un curso de MBE con metodología B-learning, como un modelo para enseñar el rol de académico.

Métodos: Se diseñó un curso equivalente a 20 horas académicas, distribuidas en 9 semanas, con 4 sesiones online de módulos de autoinstrucción y 5 sesiones presenciales integradoras. La evaluación del curso se realizó a través de tareas que evaluaban el aprendizaje de los contenidos de los módulos online y un trabajo final que consistió en una presentación de síntesis de evidencia en pequeño grupo.

Se realizó una encuesta de satisfacción a los residentes que realizaron la primera versión b-learning del curso. 
Resultados: La versión piloto del curso se implementó con los residentes de primer año de medicina de urgencia $(n=12)$. Todos respondieron la encuesta al terminar el curso. La mayoría respondió que estaba de acuerdo o muy de acuerdo en que los contenidos y metodología docente fueron adecuados y que valoraban lo aprendido para su práctica clínica. Se evaluó como adecuada la proporción entre contenido online y presencial, destacando que la sesión integradora resulta muy útil para aplicar lo aprendido y resolver dudas. Mencionan que el tener acceso a contenido teórico online previo a las sesiones presenciales y completar las tareas, les permite comprender mejor los temas y enfocar las sesiones de grupo pequeño a la aplicación práctica. El 91,7\% de los encuestados respondió que el sistema b-learning le acomoda.

Conclusión: La metodología b-learning permite a los residentes optimizar mejor el uso de su escaso tiempo y les acomoda al permitirles utilizar mejor las sesiones presenciales para resolver dudas y aplicar el contenido. Esta primera versión fue evaluada positivamente y permitió lograr su objetivo docente. Con esta modalidad esperamos poder ofrecer a esta oportunidad a un mayor número de residentes por año.

\section{Estudio de correlación de clima educativo y Burnout en residentes de medicina interna en el Uruguay}

Silvera Luciana (1), Añon Ximena(1), Bernardi Natalia (1), Carissi José María (1), Zubiaurre Valentina (1)

\section{(1) Hospital de Clínicas, Facultad de Medicina, Universidad de la República}

Introducción: El clima educativo es una forma de evaluar la calidad de los programas de residencias médicas y permite planificar modificaciones curriculares hacia una mejora de las mismas. Existen múltiples definiciones de clima educativo y en todas ellas se incluyen variados aspectos y puntos de vista. Resumiendo se podría decir que se trata de un constructo multifactorial que depende fuertemente de la percepción de los integrantes de la institución, concibiendo aspectos que van más allá de la infraestructura y el currículo, tales como los vínculos interpersonales, el currículo oculto y la cultura organizacional, entre otros (Goñi, Danza, Urgoiti, \& Durante, 2015). Existe un número importante de instrumentos disponibles para la evaluación del Clima de aprendizaje en posgrado.

Se dispone de suficiente evidencia que respalda su validez de contenido, constructo y confiabilidad. Uno de los más utilizados y validado al español es el cuestionario Dutch Residency Educational Climate Test (DRECT) (Dominguez, 2018).
Por otro lado, múltiples informes que describen una alta prevalencia de indicadores de tensión emocional, tanto entre los estudiantes de medicina como entre los residentes. Este tipo de manifestaciones de la conducta se describe como el síndrome de desgaste profesional o burnout (BO) (Julian \& Eduardo, 2014).

En Uruguay no existen estudios de correlación de clima educativo y BO en residentes, sin embargo, en el año 2013 se realizó un estudio de correlación en el Internado obligatorio, mostrando entre otras conclusiones una correlación negativa entre Clima Educativo y $\mathrm{B}$ (Goñi, Danza, Urgoiti, \& Durante, 2015).

En relación al contexto en que fue desarrollado el trabajo la realidad actual de la formación de postgrados en Uruguay la Universidad de la República es la institución con mayor responsabilidad sobre la educación universitaria en Uruguay. Sus funciones esenciales son la docencia, la extensión y la investigación, y tiene como características distintivas su autonomía, el cogobierno, la gratuidad y la laicidad, que determinan su funcionamiento.

Actualmente la Escuela de Graduados ofrece 50 carreras de especialización, discriminadas en especializaciones de inserción primaria y especializaciones de inserción secundaria.

La especialización en Medicina Interna, ésta dentro de las mismas condiciones. En el año 2018 en el país contamos con un total de 70 residentes de Medicina Interna, de los mismos el 96\% la realizan en el subsector público y el $4 \%$ en el sector privado. En lo que respecta a la distribución territorial más del $90 \%$ se concentran en la capital del país en Montevideo y el resto en el interior del país. El objetivo general del estudio fue correlacionar clima educativo y síndrome de burnout en los residentes de Medicina Interna de Uruguay.

Los objetivos secundarios son: establecer prevalencia de síndrome de burnout y conocer la media de clima educativo. Correlacionar año de residencia con clima educativo y burnout. Correlacionar dimensiones del cuestionario de burnout (agotamiento emocional, despersonalización y realización personal) con dimensiones del cuestionario sobre clima educativo (Atmosfera educacional, trabajo adaptado a competencias del residente, trabajo en equipo, coaching, evaluación y asesoramiento, educación formal, accesibilidad a los supervisores, colaboración entre pares, alta y pase de pacientes). También se planteó como objetivo analizar diferencias entre residentes de instituciones públicas y privadas.

Métodos: Se realizó una encuesta utilizando dos cuestionarios validados para dicho fin; el Dutch Residency Educational Climate Test (D-RECT) para evaluar clima educativo y Maslach Burnout Inventory $(\mathrm{MBI})$ para evaluar burnout. Los mismos fueron enviados 
a los residentes de Medicina Interna con cargo actual en el ámbito público y privado vía correo electrónico en forma anónima a través del programa Survey Monkey durante el mes de setiembre de 2018. El análisis estadístico se realizó a través del coeficiente de correlación de Spearman.

Resultados: La encuesta fue completada por $61 / 70$ (87\%) residentes. La prevalencia de burnout encontrada fue $63.93 \%$, sin diferencia entre sexos. La media del clima educativo fue de 76.49171. EI $65.52 \%$ de los residentes que presentaron burnout percibieron un clima educativo por debajo de la mediana. Se comprobó una correlación negativa estadísticamente significativa entre clima educativo y burnout ( $p: 0.000$ ). No se encontró una diferencia

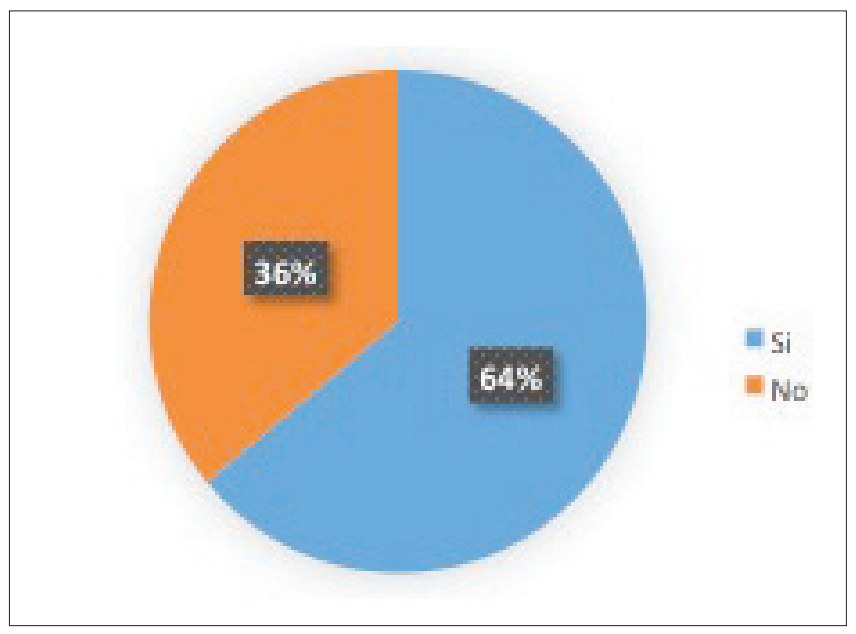

Gráfico 1. Prevalencia de burnout. estadísticamente significativa entre año de residencia y burnout ( $p$ : 0.8871 ), año de residencia y clima educativo ( $p: 0.8359)$ así como tampoco entre trabajo fuera de residencia y burnout ( $p: 0.2462$ ). En el análisis de la correlación entre dimensiones se encontró una relación estadísticamente significativa en las siguientes: correlación negativa entre agotamiento emocional - trabajo adaptado a las competencias del residente $(p=0.000)$; y entre agotamiento emocional - acceso a supervisores $(p=0.000)$. Además se observó una correlación positiva entre realización personal-trabajo adaptado a la competencia del residente $(p=0.0018)$ y realización personal-colaboración entre pares $(p=0.0497)$. No se logró analizar diferencias entre el ámbito público y privado dado el bajo número de residentes en este último.

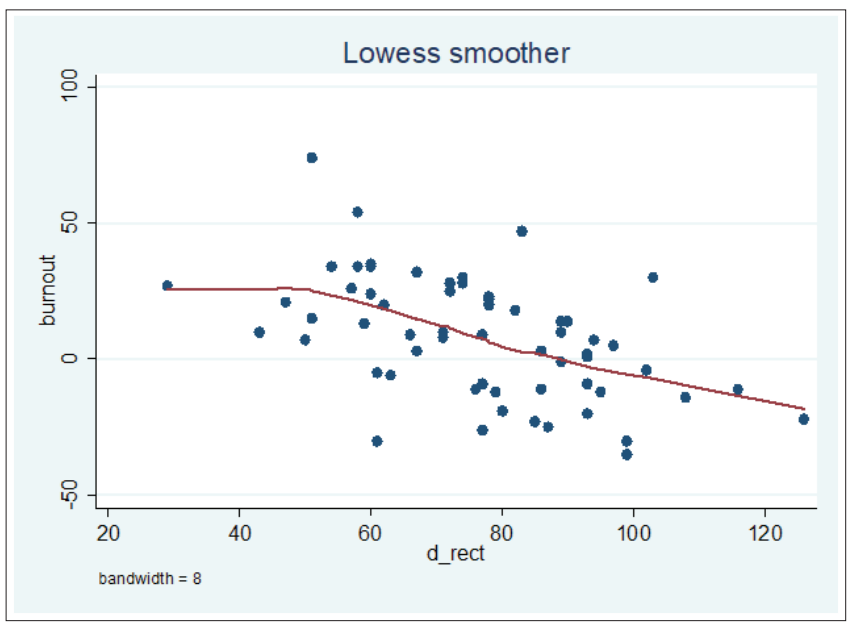

Gráfico 2. Correlación entre clima educativo y burnout.

Tabla 1- Correlación entre dimensiones de Clima Educativo y burnout. Coeficiente de Rho de Spearman.

\begin{tabular}{|c|c|c|c|c|c|c|c|c|}
\hline $\begin{array}{l}\text { BURNOUT / CLIMA } \\
\text { EDUCATIVO }\end{array}$ & $\begin{array}{l}\text { Atmósfera } \\
\text { educacional }\end{array}$ & $\begin{array}{l}\text { Trabajo adapta- } \\
\text { do a competen- } \\
\text { cia de residente }\end{array}$ & $\begin{array}{l}\text { Accesibilidad a } \\
\text { los supervisores }\end{array}$ & $\begin{array}{l}\text { Colaboración } \\
\text { entre pares }\end{array}$ & $\begin{array}{c}\text { Alta y pase de } \\
\text { pacientes }\end{array}$ & $\begin{array}{l}\text { Trabajo } \\
\text { en equipo }\end{array}$ & $\begin{array}{l}\text { Coaching, } \\
\text { evaluación y } \\
\text { asesoramiento }\end{array}$ & $\begin{array}{c}\text { Educación } \\
\text { formal }\end{array}$ \\
\hline $\begin{array}{l}\text { Agotamiento emo- } \\
\text { cional }\end{array}$ & & -0.613 & -0.589 & & -0.305 & & & \\
\hline Despersonalización & & & & & & & & \\
\hline $\begin{array}{l}\text { Realización } \\
\text { personal }\end{array}$ & & 0.3909 & & & & & & 0.3146 \\
\hline
\end{tabular}


Conclusión: Se encontró alta prevalencia de síndrome de burnout y una media de clima educativo que orienta a un clima más negativo que positivo. También se observó una correlación negativa entre percepción de clima educativo y burnout en los residentes de Medicina Interna en Uruguay. El trabajo adaptado a las competencias del residente aparece como un elemento importante favoreciendo la realización personal y en oposición al agotamiento emocional. Identificar fortalezas y debilidades del clima educativo, es una herramienta de utilidad para mejorar la calidad de los programas de formación de postgrados y trabajar en la prevención de burnout en los residentes de medicina interna.

\section{¿Es posible evaluar roles intrínsecos CanMEDS utilizando un Examen Clínico Objetivo Estructurado?}

Garrido Francisco(1), Triviño Ximena (2), Henríquez Hector (3) Cikutovic Pablo(1), Arau Raúl (4), Huete Álvaro (1)

(1) Departamento de Radiología y Diagnóstico por Imágenes, Escuela de Medicina, Pontificia Universidad Católica de Chile. (2)Dirección Académica de Docencia en Salud, Universidad San Sebastián. (3)Departamento de Radiología, Clínica Santa María, Santiago, Chile. (4)Unidad Docente Asociada Complejo Asistencial Dr. Sotero de Río, Escuela de Medicina, Pontificia Universidad Católica de Chile.

Introducción: Los jefes de programas de residencias médicas reportan dificultades en evaluar las competencias CanMEDS diferentes al Médico Experto. La evaluación de todo el espectro de competencias CanMEDS es requisito para optar a la acreditación internacional por parte del Royal College of Physicians and Surgeons of Canada. Nuestro programa de especialidad en Radiología aplica hace más de una década un Examen Clínico Objetivo Estructurado (ECOE) en el $2^{\circ}$ año de residencia, con el objetivo de certificar la adquisición de competencias en Radiología de Urgencias previo a la incorporación de los residentes a los turnos nocturnos como residente único supervisado. El propósito de esta intervención educacional es diseñar e incorporar estaciones al ECOE de Radiología de Urgencias que evalúen roles CanMEDS diferentes al Médico Experto.

Métodos: Se seleccionaron dos competencias CanMEDS (Comunicador y Colaborador) para incorporar al ECOE. A partir de estas competencias, se seleccionaron objetivos específicos que tributan a ellas a través de una encuesta a los docentes del programa. Las estaciones diseñadas fueron incorporadas al instrumento de evaluación aplicado el año 2017. Se calcularon porcentajes de logro por estación y por tipo de pauta de evaluación. Para evaluar la implementación de las estaciones, se diseñó y aplicó una encuesta de percepción a los residentes enviada en formato digital.
Resultados: Se diseñaron tres estaciones, dos para el rol Comunicador y una para el rol Colaborador. La validez de contenido se aseguró a través un panel de expertos que determinó los contenidos específicos para cada estación. Los porcentajes de logro global en las estaciones fluctuaron entre $74,7 \%$ y $95,6 \%$. Los residentes evaluaron satisfactoriamente las nuevas estaciones, además de valorar la incorporación de competencias diferentes a lo cognitivo en el instrumento de evaluación.

\begin{tabular}{|c|c|c|c|}
\hline & Estación Nº1 & Estación $\mathrm{N}^{\circ} 2$ & Estación $\mathrm{N}^{\circ} 3$ \\
\hline Rol CanMEDS & Colaborador & Comunicador & Comunicador \\
\hline $\begin{array}{l}\text { Competencia } \\
\text { clave }\end{array}$ & $\begin{array}{l}\text { Trabajar efectiva- } \\
\text { mente con otros } \\
\text { médicos y profe- } \\
\text { sionales de la salud }\end{array}$ & $\begin{array}{l}\text { Redactar el informe } \\
\text { radiológico demanera } \\
\text { oportuna, priorizando } \\
\text { aquellos exámenes } \\
\text { que contengan ha- } \\
\text { llazgos críticos o que } \\
\text { requieran un manejo } \\
\text { específico o inmediato } \\
\text { por parte de los médi- } \\
\text { cos de urgencia }\end{array}$ & $\begin{array}{l}\text { Compartir con } \\
\text { los pacientes y } \\
\text { sus familias infor- } \\
\text { mación y planes } \\
\text { respecto de la } \\
\text { atención de salud. }\end{array}$ \\
\hline $\begin{array}{l}\text { Actividad a } \\
\text { realizar }\end{array}$ & $\begin{array}{l}\text { Recomendaral clínico } \\
\text { un estudio de imáge- } \\
\text { nes porvía telefónica } \\
\text { para una paciente } \\
\text { embarazada }\end{array}$ & $\begin{array}{l}\text { Producir un informe } \\
\text { radiológico }\end{array}$ & $\begin{array}{l}\text { Explicar a una ma- } \\
\text { dre la condición } \\
\text { clínica de su hijo }\end{array}$ \\
\hline Contenido & $\begin{array}{l}\text { Radiología Abdo- } \\
\text { minal - Apendicitis } \\
\text { Aguda }\end{array}$ & $\begin{array}{l}\text { Radiología Múscu- } \\
\text { lo-esquelética-Com- } \\
\text { plicaciones de una } \\
\text { fractura costal. }\end{array}$ & $\begin{array}{l}\text { Radiología Pediá- } \\
\text { trica - Invagina- } \\
\text { ción intestinal }\end{array}$ \\
\hline $\begin{array}{l}\text { Paciente } \\
\text { estandarizado }\end{array}$ & $\begin{array}{l}\text { Sí (médico emer- } \\
\text { genciólogo) }\end{array}$ & No & Sí (madre) \\
\hline
\end{tabular}

Conclusión: El ECOE es un instrumento de evaluación del tercer nivel de la pirámide de Miller que permite certificar roles CanMEDS diferentes al Médico Experto, como el rol Comunicador y Colaborador. La incorporación de estas estaciones de ECOE amplía el espectro de competencias evaluadas durante la residencia.

\section{Necesidad de supervisión en actividades profesionales confiables al inicio de las residencias médicas: ¿Cuál es la percepción de los residentes y sus docentes?}

Carozza Maria (1)(2), Luiz Milena (1)(2), Silva Carolina (1)(2), Eymann Alfredo (1)(2), Llera Julián (1)(2)

(1) Servicio de Clínica Pediátrica, (2) Instituto Universitario, Hospital Italiano de Buenos Aires, Argentina

Introducción: En 2014 la Asociación Americana de Universidades Médicas, propuso una lista de actividades profesionales confiables (APROC) que los graduados de medicina deberían ser capaces de realizar en su primer dia de residencia sin supervisión directa. Las APROC no están claramente definidas en nuestro país, lo que genera dificultades para establecer niveles de supervisión adecuados 
para los médicos ingresantes al sistema de residencias. Además, no existen datos en nuestro medio sobre la percepción de necesidad de supervisión en estas actividades. El objetivo del presente trabajo fue evaluar y comparar la percepción de residentes y sus docentes acerca del nivel de supervisión que requieren para realizar APROC.

Métodos: Estudio de corte transversal, realizado durante septiembre y octubre de 2018. Se incluyeron residentes de primer año de especialidades clínicas y quirúrgicas y sus docentes. Se enviaron encuestas electrónicas o en papel, con participación voluntaria y anónima. Se preguntó sobre la percepción del nivel de supervisión que requerían los médicos ingresantes duranteel primer mes de formación, para 13 APROC.

Resultados: Se evaluaron las respuestas de 71/87 residentes y 39/68 docentes, de las 5 especialidades: cirugía (24\%), clínica médica (39\%), ginecología (9\%), pediatría (22\%) y medicina familiar (6\%). El $54 \%$ eran varones, y la mediana de edad en años fue de 26 (24-39) entre los residentes y 41 (29-60) para sus docentes. En la Tabla 1, se muestra la percepción de residentes y docentes respecto del nivel de supervisión que requerían para cada APROC los médicos ingresantes. Se observaron diferencias estadísticamente significativas, entre la percepción de residentes y docentes en 11 de las 13 APROC.

Tabla 1: Percepción de nivel de supervisión requerido por residentes y sus docentes

\begin{tabular}{|c|c|c|c|}
\hline \multirow{2}{*}{$\begin{array}{l}\text { Actividades Profesionales } \\
\text { Confiables }\end{array}$} & \multicolumn{2}{|c|}{$\begin{array}{l}\text { Percepción de auto- } \\
\text { nomía del residente }\end{array}$} & \multirow[b]{2}{*}{$\mathbf{p}$} \\
\hline & $\begin{array}{c}\text { Residen- } \\
\text { tes (\%) } \\
n=71\end{array}$ & $\begin{array}{c}\text { Docen- } \\
\text { tes }(\%) \\
n=39\end{array}$ & \\
\hline 1-Realizar una historia clínica y una exploración física & 70 & 33 & $<0.001$ \\
\hline $\begin{array}{l}\text { 2- Priorizar un diagnóstico diferencial después } \\
\text { de una consulta clínica }\end{array}$ & 35 & 13 & 0.012 \\
\hline $\begin{array}{l}\text { 3- Recomendar e interpretar pruebas diagnósticas } \\
\text { y de pesquisa rutinarias }\end{array}$ & 45 & 5 & $<0.001$ \\
\hline $\begin{array}{l}\text { 4- Elaborar y discutir órdenes y prescripciones } \\
\text { médicas }\end{array}$ & 34 & 8 & 0.002 \\
\hline $\begin{array}{l}\text { 5- Documentar la consulta en la historia clínica } \\
\text { del paciente }\end{array}$ & 73 & 44 & 0.002 \\
\hline $\begin{array}{l}\text { 6- Hacer una presentación oral de una consulta } \\
\text { clínica }\end{array}$ & 54 & 21 & 0.001 \\
\hline $\begin{array}{l}\text { 7-Formular interrogantes clínicosy obtener evidencia } \\
\text { para mejorar la atención médica del paciente }\end{array}$ & 61 & 59 & 0.871 \\
\hline $\begin{array}{l}\text { 8-Daro recibirun traspaso de cuidado de un paciente } \\
\text { (pase de guardia) }\end{array}$ & 3 & 13 & 0.005 \\
\hline $\begin{array}{l}\text { 9- Colaborar como miembro de un equipo } \\
\text { interprofesional }\end{array}$ & 69 & 36 & 0.001 \\
\hline $\begin{array}{l}\text { 10-Reconocer a un paciente que necesite atención } \\
\text { de urgencia e iniciar su evaluación y manejo }\end{array}$ & 3 & 5 & 0.003 \\
\hline $\begin{array}{l}\text { 11- Obtener consentimiento informado para } \\
\text { pruebas o procedimientos }\end{array}$ & 75 & 23 & $<0.001$ \\
\hline $\begin{array}{l}\text { 12- Realizar procedimientos médicos generales } \\
\text { ( RCP básica, ventilación con bolsa y máscara, } \\
\text { técnica estéril, venopuntura y colocación de vía } \\
\text { periférica, colocación de sonda vesical) }\end{array}$ & 31 & 0 & $<0.001$ \\
\hline $\begin{array}{l}\text { 13- Identificar las fallas del sistema y contribuir } \\
\text { a una cultura de seguridad y mejora }\end{array}$ & 55 & 41 & 0.163 \\
\hline
\end{tabular}

Conclusión: Los médicos residentes percibieron requerir menor nivel de supervisión en comparación con sus docentes en todas las APROC. Sin embargo solo se sienten capaces de realizar la mitad de ellas de forma autónoma, mientras que sus docentes perciben que requieren supervisión directa en la mayoría. Ambos grupos presentaron percepciones similares sólo en relación a la capacidad de los residentes de identificar errores del sistema, así como de formular interrogantes clínicos y responderlos. Surge la importancia de mejorar la evaluación de las competencias de los recién graduados, para definir niveles de supervisión basados en APROC con mayor precisión.

\section{Evaluación de la Asignatura de Neurociencias por Residentes de Psiquiatría}

Rubí Patricia (1), Bustos Claudio(1), Valdivia Mario(1), VergaraBarra Pablo(1), Rocha Marcela(1).

(1) Departamento de Psiquiatría y Salud Mental, Facultad de Medicina, Universidad de Concepción, Chile

Introducción: La formación en Neurociencias aplicadas a la Psiquiatría es considerada fundamental por los directores de los programas de especialización ${ }^{1-3}$ y residentes ${ }^{4}$. El currículum de los cursos de formación debe adecuarse a los requerimientos de la práctica ${ }^{5}$, para lo cual es necesario conocer la evaluación que de él realizan los estudiantes. En general, la medición de satisfacción y calidad de un programa de formación en neurociencias se realiza respecto de la asignatura en su totalidad ${ }^{6-8}$, siendo menos frecuente la medición de módulos individuales 9 .

Hace 3 años surge la asignatura semestral de Neurociencias para residentes de primer año de los Programas de Especialización en Psiquiatría Adultos y Psiquiatría del Niño y del Adolescente de la Universidad de Concepción (Chile). Ésta pretende entregar conocimientos de Neurociencias aplicados a la clínica psiquiátrica. Por primera vez esta asignatura es sometida a un proceso de evaluación con el objetivo de definir fortalezas y aspectos deficientes susceptibles de mejorar. La asignatura consta de 17 clases que comprenden diversos temas de esta área (Tabla No1), impartidas por especialistas en Neurología, Psiquiatría, Neurofisiología, Psicología, Genética y Neurorradiología.

El objetivo de este estudio fue evaluar las clases de la asignatura de Neurociencias impartida a residentes de Psiquiatría, en cuanto a cantidad de contenidos entregados, metodología de enseñanza y pertinencia de la evaluación. 
Método: Se realizaron encuestas online a los 13 residentes de primer año de los programas de especialización de Psiquiatría de Adultos y del Niño y del Adolescente, que recientemente cursaron la asignatura de Neurociencias (primer semestre de 2018), de manera confidencial. Se consultó, para cada una de las 17 clases de la asignatura en cuanto a: 1) Cantidad de contenidos entregados en clase, 2) Calidad de la metodología de enseñanza, 3) Pertinencia de la evaluación respecto de los contenidos entregados y 4) Preguntas abiertas con comentarios y sugerencias.

Resultados: La evaluación de cantidad de contenidos y calidad de la metodología presenta un nivel de acuerdo interjueces suficiente (kappa >0,4), no así la pertinencia de la evaluación de acuerdo a los contenidos entregados (kappa=0.17) (Figura №1).

Según los residentes que cursaron la asignatura, el $47 \%$ de las clases tiene cantidad de contenidos adecuados. Las clases de neurodesarrollo y envejecimiento/neurodegeneración resultaron con contenidos en cantidad excesiva para el $92 \%$ de los alumnos. La clase de neuroanatomía resultó tener contenidos insuficientes para el $77 \%$ de los residentes (Figura №1).

En relación a la calidad metodológica de las clases, 8 de 17 clases fueron catalogadas con promedios en el rango de adecuadas o completamente adecuadas y 4 clases con promedios en el rango de inadecuadas o completamente inadecuadas (Figura №1).
En relación a la evaluación de contenidos, 1/3 de las clases presenta una evaluación pertinente de acuerdo a los contenidos entregados. Solo dos clases se consideraron no adecuadas a la evaluación (Figura №1).

Tabla 1: Clases impartidas en la asignatura de Neurociencias

\begin{tabular}{l}
\hline Anatomía clínica del Sistema Nervioso Central \\
\hline Funciones cerebrales \\
\hline Neurodesarrollo \\
\hline Neurotransmisión, sistema neuroendócrino, plasticidad neuronal, \\
neuromodulación \\
\hline Evolución del cerebro y la conducta \\
\hline Genética y epigenética y su relación con los trastornos mentales \\
\hline Neurobiología de la depresión \\
\hline Neurobiología del T. Bipolar \\
\hline Neurobiología de la Esquizofrenia y otras psicosis \\
\hline Neurociencia cognitiva \\
\hline Ciclo sueño vigilia y neurobiología de los T. Del sueño \\
\hline Neurobiología de la ansiedad y T. ansiosos \\
\hline Neurobiología del TDAH y trastornos del espectro autista \\
\hline Neurobiología de las adicciones \\
\hline Envejecimientoyneurodegeneración. Bases neurofisiológicas delas demencias \\
\hline Evaluación neuropsicológica \\
\hline Neurorradiología \\
\hline
\end{tabular}

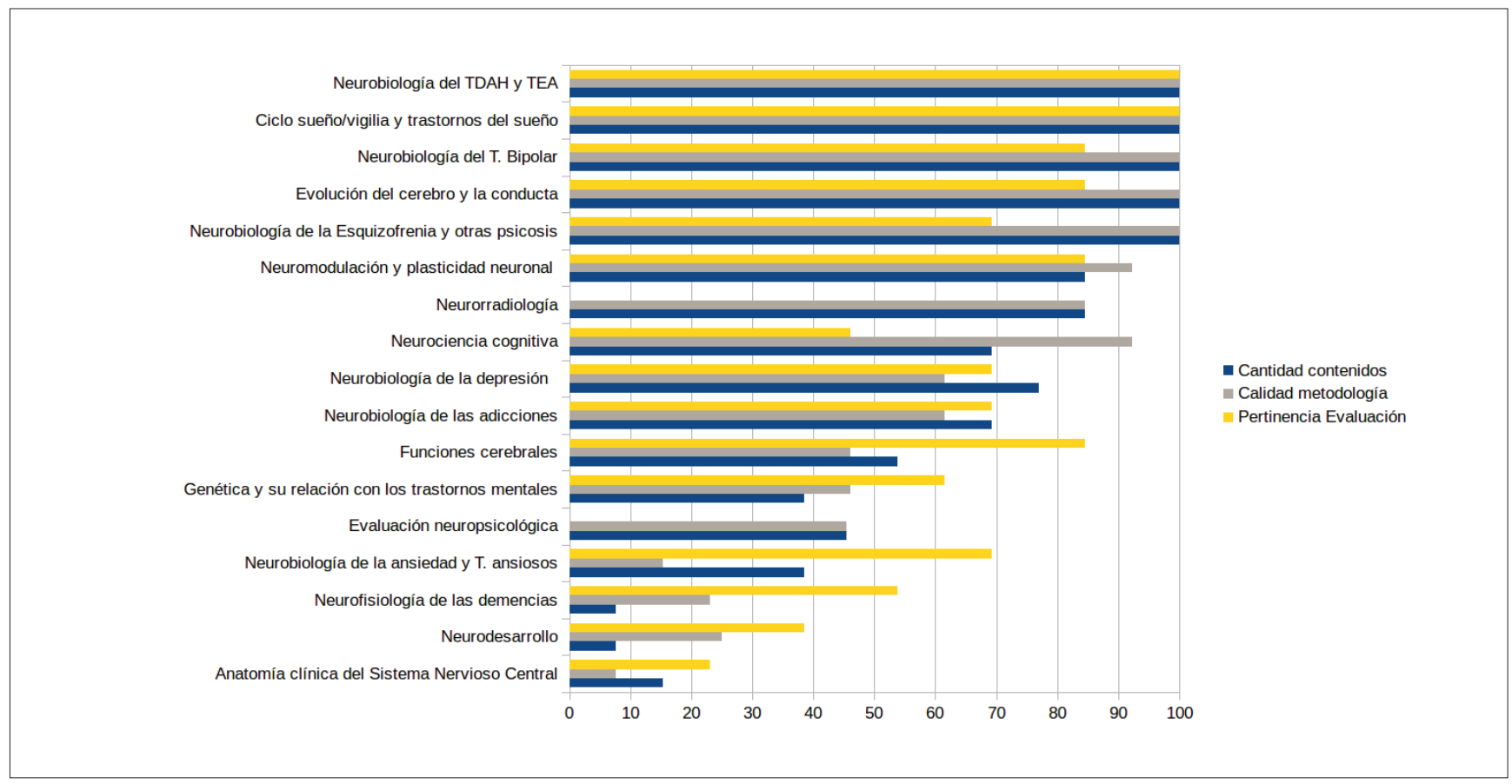

Figura 1: Cantidad de contenidos, calidad metodológica y pertinencia de la evaluación de las clases de la asignatura de Neurociencias 
Conclusión: Es posible evaluar las clases de esta asignatura en sus distintos aspectos de manera confiable, al menos en lo que respecta a cantidad de contenidos y calidad de la metodología de enseñanza. Se requiere mejorar la evaluación de la pertinencia de la evaluación de contenidos.

\section{Referencias:}

Benjamin S. Educating psychiatry residents in neuropsychiatry and neuroscience. Int Rev Psychiatry. 2013; 25(3): 265-275.

Benjamin S, Widge A, Shaw K. Neuropsychiatry an Neuroscience Milestones for General Psychiatry Trainees. Acad Psychiatry. 2014; 38: 275-282.

Chung JY, Insel TR. Mind the Gap: Neuroscience Literacy and the Next Generation of Psychiatrists. Acad Psychiatry. 2014; 38: 121-123.

Fung LK, Akil M, Widge A, Roberts LW, Etkin A. Attitudes Toward Neuroscience Education in Psychiatry: a National Multi-stakeholder Survey. Acad Psychiatry. 2015; 39: 139-146.

Roffman JL, Simon AB, Prasad KM, Truman CJ, Morrison J, Ernst CL. Neuroscience in Psychiatry Training: How Much Do Residents Need to Know?. Am J Psychiatry. 2006; 163: 919-926.

Dunstone DC. A Neurosciences-in-Psychiatry Curriculum Project for Residents in Psychiatry. Acad Psychiatry. 2010; 34: 31-38.

Gopalan P, Azzam PN, Travis MJ, Schlesinger A, Lewis DA. Longitudinal Interdisciplinary Neuroscience Curriculum. Acad Psychiatry. 2014; 38(2): 163-167.

Griffith JL. Neuroscience an Humanistc Psychiatry: a Residency Curriculum. Acad Psychiatry. 2014; 38: 177-184.

Ross DA, Rohrbaugh R. Integrating Nauroscience in the Training of Psychiatrists: A Patient-Centered Didactic Curriculum Based on Adult Learning Principles. Acad Psychiatry. 2014; 38(2): 154-162.

\section{Incorporación y desarrollo de objetivos de formación CanMEDS en la Pontificia Universidad Católica de Chile}

Bravo Muriel(1), Calderón Jorge(1), Abarca Katia (1)

(1) Pontificia Universidad Católica de Chile, Santiago, Chile

Introducción: En la actualidad, la práctica médica exige el desarrollo de diversas competencias en la formación de un médico especialista. Esto implica revisar y actualizar los procesos de en- señanza-aprendizaje a estas nuevas necesidades para garantizar la formación integral y el correcto ejercicio de la profesión de los futuros médicos. Con este propósito, la Pontificia Universidad Católica de Chile ha iniciado un proceso de incorporación y desarrollo de los objetivos de formación del modelo CanMEDS del Royal College of Physicians and Surgeons of Canada (RCPSC) en los Programas de Especialidades Médicas, uno de los modelos más utilizados en el mundo en formación de residentes y un ejemplo en calidad de educación. Debido a lo anterior, el objetivo de este trabajo es desarrollar una metodología que permita la incorporación y desarrollo de estos objetivos de formación en los Programas de Especialidades Médicas dentro de un proceso de mejora continua.

Métodos: Este proyecto ha involucrado un plan de trabajo progresivo en dos programas de especialidad médica: Pediatría y Psiquiatría, con los siguientes procesos comunes:

- Análisis de objetivos, contenidos e indicadores de formación RCPSC

- Revisión de documentación oficial de las actividades académicas

- Distribución de objetivos de formación en base al año del Programa

- Detección y consulta de objetivos y contenidos que necesitan mayor desarrollo

- Ordenamiento de objetivos por actividad académica

- Revisión y edición de Programas de actividades según objetivos de formación

- Rediseño de estrategias, metodologías y evaluación centrado en los procesos de aprendizaje

Resultados: Este proceso ha originado dos metodologías de incorporación y desarrollo de los objetivos del modelo CanMEDS diferentes y centradas en la cobertura total de estos objetivos en las distintas actividades académico-clínicas de los programas y en su desarrollo progresivo en base al año de formación. La diferencia principal entre ellas es su punto de partida, ya que mientras una parte desde los mismos objetivos generales y los distribuye por año de formación, la otra lo hace desde el desglose detallado de los contenidos y habilidades de la competencia Médico Experto y los relaciona con las estrategias de aprendizajes utilizadas en las diversas actividades del programa.

Conclusión: Ambas metodologías implementadas han sido evaluadas positivamente por todos los actores involucrados, quienes lo consideran un aporte y avance significativo en los procesos de enseñanza-aprendizaje de la medicina. La incorporación de objetivos a través de estas metodologías, ha permitido reorientar la formación para cumplir con estándares internacionales, detectar áreas de mejora, asegurar un proceso de enseñanza-aprendizaje gradual y coherente desde los objetivos 
de aprendizaje hasta la evaluación, e innovar en torno a las prácticas educativas que se desarrollan en la UC. Este proceso no sería posible sin el trabajo continuo y comprometido de la comunidad académica. Dentro de los principales desafíos encontrados, están la formación en el modelo de todos los docentes, la cobertura de todos los contenidos, la elección y diseño de instrumentos de aprendizaje y evaluación que permitan realizar un proceso efectivo.

\section{¿Cómo enseñar y evaluar los Roles CanMEDS? Diseño e implementación de un curso dirigido a docentes}

Parra Claudia'; Riquelme Catalina'; Pinedo José'; Hoyl Trinidad'; Cifuentes Lorena'

(1) Dirección de Postgrado, Escuela de Medicina, Pontificia Universidad Católica de Chile, Santiago, Chile

Introducción: Desde el año 2010, la Dirección de Postgrado de la Escuela de Medicina de la Pontificia Universidad Católica de Chile, ha incorporado en la formación de residentes el modelo de competencias CanMEDS del Royal College of Physicians and Surgeons of Canada (RCPSC). Este modelo propone que para la entrega de una atención en salud de alta calidad y segura, los médicos deben desarrollar los siguientes Roles: Médico Experto, Académico, Colaborador, Comunicador, Líder, Promotor de la Salud y Profesional.

En este contexto, se ha detectado la necesidad de promover el faculty development, es decir, llevar a cabo actividades para que los docentes desarrollen las competencias necesarias para enseñar y evaluar el desempeño de los residentes en cada uno de los Roles CanMEDS. Este proceso también permitiría a los docentes capacitarse para desarrollar ellos mismos estas competencias y ser modelos frente a los residentes a través de su propio desempeño. Con este objetivo se ha diseñado, producido e implementado un curso semipresencial para docentes, enfocado en las competencias necesarias para enseñar y evaluar cada uno de los Roles CanMEDS en la formación de residentes de la Escuela de Medicina UC.

Métodos: Para crear el curso en metodología semipresencial se llevó a cabo un proceso continuo y estandarizado que incluyó las siguientes etapas: diseño, producción, implementación y evaluación. Durante la fase de diseño primero se estructuró el diseño instruccional de cada clase online y de los talleres, partir del material desarrollado por el RCPSC y la Universidad de Ottawa. En la fase de producción, en conjunto con profesores expertos en cada uno de los Roles, se grabaron las clases online y se desarrollaron las tareas y materiales que se ocuparían en los talleres. Para la fase de implementación se requirió una tutoría administrativa junto con una tutoría académica. Durante la fase de evaluación se aplicó una encuesta de satisfacción para determinar los cambios que se requerirían aplicar en el curso, dentro de un proceso de mejora continua.

Resultados: El curso se realizó en su primera versión en Septiembre del año 2018, y contó con 26 docentes pertenecientes a los programas de Pediatría y Medicina Interna. El curso consistió en 10 sesiones ( 6 clases online y 4 talleres presenciales). Cada clase online abordó uno de los Roles CanMEDS (a excepción de Médico Experto, ya que está mayormente relacionado con competencias clínicas, y el curso se enfocó en las competencias transversales). En cada video de las clases se describieron los Roles y se entregaron tips para su enseñanza y evaluación. En cada clase los docentes respondieron una tarea online individual de aplicación y reflexión. En los talleres presenciales se realizaron actividades prácticas para aplicar las competencias aprendidas.

Se implementó un sistema de tutoría administrativa, dirigido por dos coordinadoras quienes gestionaron la administración de la plataforma de las clases online. Además, se realizó una tutoría académica, a partir de la cual se realizó una revisión general (no personalizada) de las tareas, para así retomar los principales temas abordados por los docentes en los talleres presenciales.

Al finalizar el curso se aplicó una encuesta de satisfacción, que en total respondieron 22 participantes. La encuesta tipo Likert (1=muy en desacuerdo, 4=muy de acuerdo) consistió en 13 ítems que evaluaron la satisfacción de los docentes con los distintos componentes del curso. Como principales resultados los docentes reportaron que aprendieron sobre estrategias de enseñanza y evaluación de los Roles CanMEDS (18.2\% de acuerdo, 81.8\% muy de acuerdo), señalaron que han logrado reflexionar sobre la importancia de su rol como docentes en el modelaje de cada uno de los Roles (9.1\% de acuerdo, $90.9 \%$ muy de acuerdo), y mencionaron que podrán aplicar lo aprendido en su rol como docentes ( $40.9 \%$ de acuerdo, $59.1 \%$ muy de acuerdo). Por otra parte, los docentes indicaron que la metodología semipresencial fue efectiva para su formación en los contenidos del curso (13.6\% de acuerdo, $86.4 \%$ muy de acuerdo), y que recomendarían el curso a otros colegas ( $9.1 \%$ de acuerdo, $90.9 \%$ muy de acuerdo).

Dentro de los comentarios que escribieron en la encuesta de satisfacción y que manifestaron verbalmente en el último taller, se encuentra nuevamente su alta satisfacción con el curso, pero con algunas sugerencias para mejorar las tareas y su feedback. 
Tabla 1. Resultados Encuesta de Satisfacción del Curso

\begin{tabular}{|c|c|c|c|c|}
\hline $\begin{array}{l}\text { En una escala del } 1 \text { al } 4 \text {, indique el grado de acuerdo respecto a las siguientes } \\
\text { afirmaciones: }\end{array}$ & $\begin{array}{l}\text { Muy en des- } \\
\text { acuerdo }\end{array}$ & $\begin{array}{c}\text { En } \\
\text { desacuerdo }\end{array}$ & $\begin{array}{c}\text { De } \\
\text { acuerdo }\end{array}$ & $\begin{array}{l}\text { Muy de } \\
\text { acuerdo }\end{array}$ \\
\hline $\begin{array}{l}\text { Este curso me ha permitido aprender sobre estrategias para la enseñanza y evaluación } \\
\text { de los Roles CanMEDS en la formación de residentes. }\end{array}$ & & & $18,2 \%$ & $81,8 \%$ \\
\hline $\begin{array}{l}\text { El curso me ha permitido reflexionar sobre la importancia de mi rol como docente } \\
\text { en el modelaje de cada uno de los Roles CanMEDS en la formación de los residentes. }\end{array}$ & & & $9,1 \%$ & $90,9 \%$ \\
\hline Podré aplicar lo aprendido en este curso en mi rol como docente. & & & $40,9 \%$ & $59,1 \%$ \\
\hline $\begin{array}{l}\text { Los contenidos de las clases videadas son relevantes y pertinentes a los objetivos } \\
\text { del curso. }\end{array}$ & & & $27,3 \%$ & $72,7 \%$ \\
\hline La entrega de contenidos por parte de los profesores en las clases videadas es clara. & & & $18,2 \%$ & $81,8 \%$ \\
\hline La duración de cada clase videada, en general es adecuada. & & & $50 \%$ & $50 \%$ \\
\hline $\begin{array}{l}\text { El material complementario es atingente y permite profundizar en cada uno de los } \\
\text { Roles CanMEDS. }\end{array}$ & & & $36,4 \%$ & $63,6 \%$ \\
\hline $\begin{array}{l}\text { Las tareas me han permitido realizar transferencia y aplicación concreta de los } \\
\text { contenidos del curso. }\end{array}$ & & $4,5 \%$ & $54,5 \%$ & $40,9 \%$ \\
\hline $\begin{array}{l}\text { Las actividades de los talleres presenciales me permitieron aplicar lo aprendido } \\
\text { en el curso. }\end{array}$ & & & $22,7 \%$ & $77,3 \%$ \\
\hline $\begin{array}{l}\text { La metodología semipresencial fue una estrategia efectiva para mi formación en } \\
\text { los contenidos del curso. }\end{array}$ & & & $13,6 \%$ & $86,4 \%$ \\
\hline $\begin{array}{l}\text { El tiempo que tuve que invertir para realizar las actividades de este curso fue el } \\
\text { adecuado. }\end{array}$ & & $4,5 \%$ & $27,3 \%$ & $68,2 \%$ \\
\hline La plataforma del curso (WebCursosUC) ha sido una herramienta fácil de utilizar. & & & $31,8 \%$ & $68,2 \%$ \\
\hline Recomendaría el curso de todas maneras a otros colegas. & & & $9,1 \%$ & $90,9 \%$ \\
\hline
\end{tabular}

Conclusión: Durante el proceso de implementación del marco de competencias CanMEDS, se ha hecho evidente la necesidad de que los docentes dominen este modelo para poder enseñarlo y evaluarlo de manera efectiva, y particularmente, para que sean modelos en su desempeño diario frente a los residentes y el resto del equipo de salud.

Los resultados de la primera implementación del curso han evidenciado una alta satisfacción de los docentes con las estrategias aprendidas y la metodología semipresencial. Actualmente, se está realizando una segunda versión para 21 docentes de los programas de Medicina Interna y Medicina Familiar, dentro de la cual se han incorporado cambios en las tareas y su feedback, basados en las sugerencias realizadas por los docentes que participaron en la versión del año 2018.

Se espera que el curso sea dictado nuevamente este año para otros programas, con el objetivo de seguir potenciando el desarrollo docente y facilitar la implementación del modelo CanMEDS en la práctica diaria de todo el equipo de salud.

\section{Impacto en Aprendizaje de un Sistema Electrónico de Validación de Exámenes de Función Pulmonar en Residentes}

Oyarzún I (1), Arandia V (1), Moya A (1), Brockmann PE (1), Adasme $\mathrm{R}(2)$, Caussade S (1), Bertrand P (1)

(1)Departamento Cardiología y Enfermedades Respiratorias Pediátricas, Facultad Medicina, Pontificia Universidad Católica de Chile, Chile. (2)Escuela de Kinesiología, Facultad de ciencias de la rehabilitación, Universidad Andres Bello

Introducción: La residencia de enfermedades respiratorias pediátricas contempla un período limitado de entrenamiento del residente en la interpretación de función pulmonar. Su exposición es variable y dependiente del número de exámenes y continuidad tutorial. Recientemente hemos implementado un sistema en línea de validación de informes de función pulmonar que son corregidos por un docente-tutor. 
Objetivo: Evaluar el impacto de un sistema electrónico de validación de exámenes de función pulmonar como herramienta educativa en la residencia de enfermedades respiratorias pediátricas.

Metodología: Para evaluar el sistema se aplicaron los siguientes instrumentos según los 4 niveles de evaluación de entrenamiento de Kirkpatrick: 1. Reacción: Encuesta Likert a residentes y docentes; 2. Aprendizaje: Rúbrica de concordancia de 12 puntos en informes de Espirometría utilizando al tutor como "gold standard". Se evaluó reconocimiento de normalidad o alteración (obstructiva, restrictiva, reversibilidad), aceptabilidad, esfuerzo en maniobras y formato de infome. Para análisis de concordancia usamos índice Kappa de Fleiss y clasificación de concordancia de Landis \& Koch; 3. Comportamiento: Encuesta de percepción de autonomía y seguridad para residentes y ex residentes; 4. Resultados: Análisis de tiempo de entrega de informes y exposición a exámenes.

Resultados: El sistema ha sido utilizado por 5 residentes y 5 docentes durante 6 meses. Reacción:Fue evaluado de forma positiva por el 100\% de los encuestados, el tiempo para informar fue de 2-3 horas para residentes y 1-2 horas para tutores, el cual fue considerado adecuado. El $60 \%$ consideró que la retroalimentación fue oportuna. Aprendizaje: Se analizaron 304 espirometrías categorizadas como: normal: 73\%, limitación obstructiva: $11 \%$, limitación restrictiva: $2 \%$, reversibilidad: $13 \%$ y no interpretable: $1.6 \%$. La mediana de puntos de concordancia por rúbrica fue 12 (RIC 12;12) y de 2(2;2) puntos para todos los ítems. Promedio de concordancia por binomio independiente residente-tutor 94.6\%. Índices Kappa para espirometría normal, alterada, severidad y reversibilidad fue $0.91,0.90,0.86$ y 0.79 respectivamente. Se encontró diferencia significativa $(p=0,001)$ en corrección de informes entre docentes, no así entre residentes $(p=0,1)$. Se observó caída en concordancia a los 4 y 6 meses de uso del sistema, con mejoría entre periodos. Comportamiento: El 100\% de los residentes consideró que este sistema es un complemento a su formación percibiendo mejoría en interpretación y seguridad en informe. $80 \%$ de los tutores está totalmente de acuerdo en que han reforzado sus propios conocimientos. Tutores y residentes recomendarían este sistema a otros programas. Resultados: En 6 meses cada residente se expuso en promedio a 291 28 DE espirometrías versus $61 \pm 15 \mathrm{DE}$ durante un mes de rotación. El tiempo de entrega de informe se redujo de 5 a 2 días. De 15 ex-residentes encuestados, 80\% le habría gustado un sistema similar.

Conclusiones: Este sistema electrónico de validación de exámenes demuestra satisfacción en residentes y tutores además de utilidad como herramienta de aprendizaje. Residentes no mostraron diferencias significativas en concordancia. Tutores presentan concordancia aceptable pero con variabilidad en el tiempo. Estos datos sugieren la necesidad de reforzar la retroalimentación en periodos definidos durante uso de la herramienta.

\section{Mejoramiento continuo de las competencias para manejar evidencia e investigar en el Programa de Formación de Médicos Especialistas en Medicina Físicay Rehabilitación}

Nahuelhual Paula (1)(2), Rotter Karin (1)(2), Perez,Natalia (2), Salas Inés (2)

(1)Facultad de Medicina CAS-UDD, (2)Dirección de Investigación y Desarrollo, Teletón Chile.

Introducción: El Postítulo de Especialidad Médica en Medicina Física y Rehabilitación (MF\&R) de la Universidad del Desarrollo creado con el impulso y colaboración de Teletón Chile y el Hospital Clínico Mutual de Seguridad, se inició el 2011. Dura 3 años y dentro de su curriculum consideró desde su inicio el desarrollo de competencias para investigar y el manejo de evidencia y, además, desde el punto de vista organizacional, el mejoramiento curricular continuo y un foco en el estudiante. Estas competencias integran el rol académico del modelo CanMeds para médicos.

El objetivo de este trabajo fue describir el mejoramiento contínuo del desarrollo de competencias para investigar y manejar evidencia que ha experimentado el programa de formación de médicos especialistas en MF\&R desde su creación y sus resultados preliminares.

Métodos: El postítulo tiene 4 cupos anuales, un conjunto de asignaturas tipo curso, tipo rotaciones clínicas en variados campos clínicos asistenciales y el proyecto de investigación. El equipo docente principal a cargo del desarrollo de las competencias para investigar y manejar evidencia pertenece a Teletón.

Se realiza evaluación regular de satisfacción de los estudiantes al término de los cursos y rotaciones, realización de grupos focales con estudiantes y docentes, un claustro participativo al término del año académico donde se resumen fortalezas y debilidades del programa y se seleccionan las brechas próximas a abordar.

Se propone la implementación de cursos y actividades regulares que apoyen el desarrollo de las competencias de investigación y aplicación de la evidencia científica disponible para el mejoramiento continuo de la atención médica proporcionada a los pacientes y sus familias.

Resultados: El mejoramiento continuo se realizó en tres etapas: Etapa 1 (2011-2014): Curso de medicina basada en evidencia (MBE): durante el primer año de residencia y realización de Tesis durante el tercer año de la residencia.

Un total de 11 residentes pasaron por sólo por esta etapa: 9/11 finalizaron la tesis; 0/11 presentaron en congresos nacionales/ internacionales; $5 / 11$ publicaron en revistas científicas. 
Etapa 2 (2015-1016): Rediseño de curso MBE orientado a la resolución de problemas clínicos de rehabilitación. Se agrega curso "Investigación en Rehabilitación v1"en segundo año de residencia. Etapa 3 (2017- ): Se agregan talleres de lectura crítica para los residentes, al inicio dirigidos por docentes y luego por los propios residentes en temas relevantes para la especialidad.

Un total de 8 residentes pasaron por las etapas 2 y 3 , de los cuales: $8 / 8$ finalizaron la tesis; $3 / 8$ han presentado en congresos nacionales/ internacionales y $2 / 8$ han publicado en revistas científicas.

Los docentes identifican, en los grupos focales, que la formación en medicina basada en evidencia y metodología de la investigación es un sello distintivo de los residentes del programa de MF\&R de la Universidad del Desarrollo. Además, plantean la necesidad de capacitarse como tutores de tesis y en MBE.

Conclusión: El mejoramiento continuo de las actividades orientadas al desarrollo de competencias para investigar y el manejo de evidencia, mejoraron la comunicación científica de las tesis. Además, el uso y aplicación de la evidencia es un sello distintivo de los residentes.

\section{Acompañamiento en el proceso de formación: Unidad de apoyo al residente de Medicina Familiar.}

Pérez-Wilson Patricia (1), Bustos Andrea(1), Rico Felipe (1), Villaseca Patricia (1), Alvarado Débora (2)

(1)Programa de Salud y Medicina Familiar, (2)Departamento de Salud Pública, Facultad de Medicina, Universidad de Concepción, Concepción, Chile

Introducción: La Unidad de Apoyo al Residente (UAR) se formaliza a partir de una necesidad sentida de los residentes en el marco del proceso de acreditación de la especialidad de medicina familiar el año 2015, con el propósito de apoyar y acompañar a los residentes en la integración y el desarrollo de la especialización, inserción y desarrollo en sus equipos; y para apoyar a residentes que presenten alguna dificultad académica, en el transcurso del proceso formativo.

Métodos: A) Becados: Atención directa en acompañamiento de crisis y facilitación del manejo de dificultades en el proceso formativo; Atención en dificultades de adaptación al requerimiento académico facilitando la movilización de recursos motivacionales, cognitivos y sociales; Evaluación de funciones cognitivas al ingreso de primer año para actuar anticipatoriamente, otorgando herramientas a Residentes y tutores clínicos; Derivación a psicoterapia de apoyo, articulando con prestadores externos cuando esto sea necesario. B) Tutores: Acompañamiento en rol formador: talleres y reuniones de manejo de incidentes críticos y requerimientos académicos del proceso formativo
Resultados: A) Becados: Mayoritariamente atención por solicitud espontánea y en menor medida por derivación de terceros, con incremento de solicitudes desde el 2015 (2) al 2018 (12). Los motivos de consulta han sido personales (crisis) y relacionados con la especialización (adaptación, relación con tutores, desempeño, autoeficacia y motivación). B) Tutores: Talleres en Jornadas de tutores: Incidente Crítico, Modificabilidad Estructural Cognitiva y entrenamiento en Experiencia de Aprendizaje Mediado. Asesoría en relación tutor/ residente en situaciones de crisis, adaptación al proceso formativo y abordaje de perfiles cognitivos de riesgo. Tutores y residentes señalan la UAR como otra de las distinciones de la especialización.

Conclusión: La UAR es un recurso de apoyo en la Beca, que permite canalizar inquietudes y requerimientos de residentes, tutores y equipo académico, en territorio y en oportunidades formativas. La articulación con el equipo de la beca otorga soporte y continuidad para que se constituya en una oportunidad.

\section{Impacto de un programa de formación para docentes de carreras de la salud en el contexto de la implementación del Hospital de simulación USS.}

Silva Marlova (1), Buscaglione, Roxana (1)

(1) Universidad San Sebastián, Santiago, Chile

Introducción: El escenario actual, nos evidencia una oferta académica heterogénea. Esta demanda incrementada por diversas carreras de la salud conlleva a la necesidad de contar con un cuerpo docente que pueda cubrir la formación de los futuros profesionales de los equipos de salud. El proyecto de simulación e innovación en salud de la Universidad San Sebastián contempla 4 sedes, trabajo con al menos 12 carreras del área de la salud y 14.300 estudiantes, iniciando sus funciones el año 2017. La misión es garantizar una educación de calidad en las carreras del área de la salud, a través de la implementación de la educación basada en simulación, la innovación y la investigación con un modelo de excelencia orientado a promover la seguridad de las personas en el quehacer de las diversas disciplinas y en el trabajo interprofesional. En la fase de implementación del hospital de simulación, se inició un programa de capacitación docente impartiendo tres cursos a todos los docentes de la institución, con el objetivo de aumentar sus competencias y mejorar su desempeño en el área de simulación. Fueron 3 cursos: examen clínico objetivo estructurado, paciente estandarizado y curso de instructores de simulación. El objetivo de este trabajo fue evaluar la transferencia o nivel 3 de Kirkpatrick del programa de capacitación docente. 
Métodos: Se realizó un estudio transversal. Se aplicó en mayo 2018 una encuesta en línea al total de la muestra conformada por 126 profesores que hicieron al menos uno de los cursos en enero del 2017. Se cuenta con la licencia del software de encuestas en línea Question PRO. Mediante este software se aplicó una encuesta anónima enviada a correos electrónicos que evaluó el programa de capacitación docente. Mediante Likert se interrogó por diversos aspectos que apuntaran a medir la percepción de los docentes capacitados más una sección pre y post respecto a las habilidades adquiridas, indagando en la transferencia de lo aprendido en los cursos. La encuesta permaneció tres semanas disponible con reiteración semanal.
Resultados: Del total, 126 docentes que efectuaron al menos uno de los cursos impartidos obtuvimos un $67 \%$ de respuestas a la encuesta en línea. La edad promedio fue de 40 años. De los que respondieron, el 97\% indicó que estaban de acuerdo o completamente de acuerdo en que su participación en los cursos les permitió incorporar la simulación clínica en las asignaturas que imparten actualmente. Un 34\% realizó el curso de OSCE y el 94\% de ellos está diseñando e implementando esta metodología. Un 96\% indica que está en un acuerdo moderado o está completamente de acuerdo en que después de su participación en este programa mejoró su desempeño general como docente hoy.
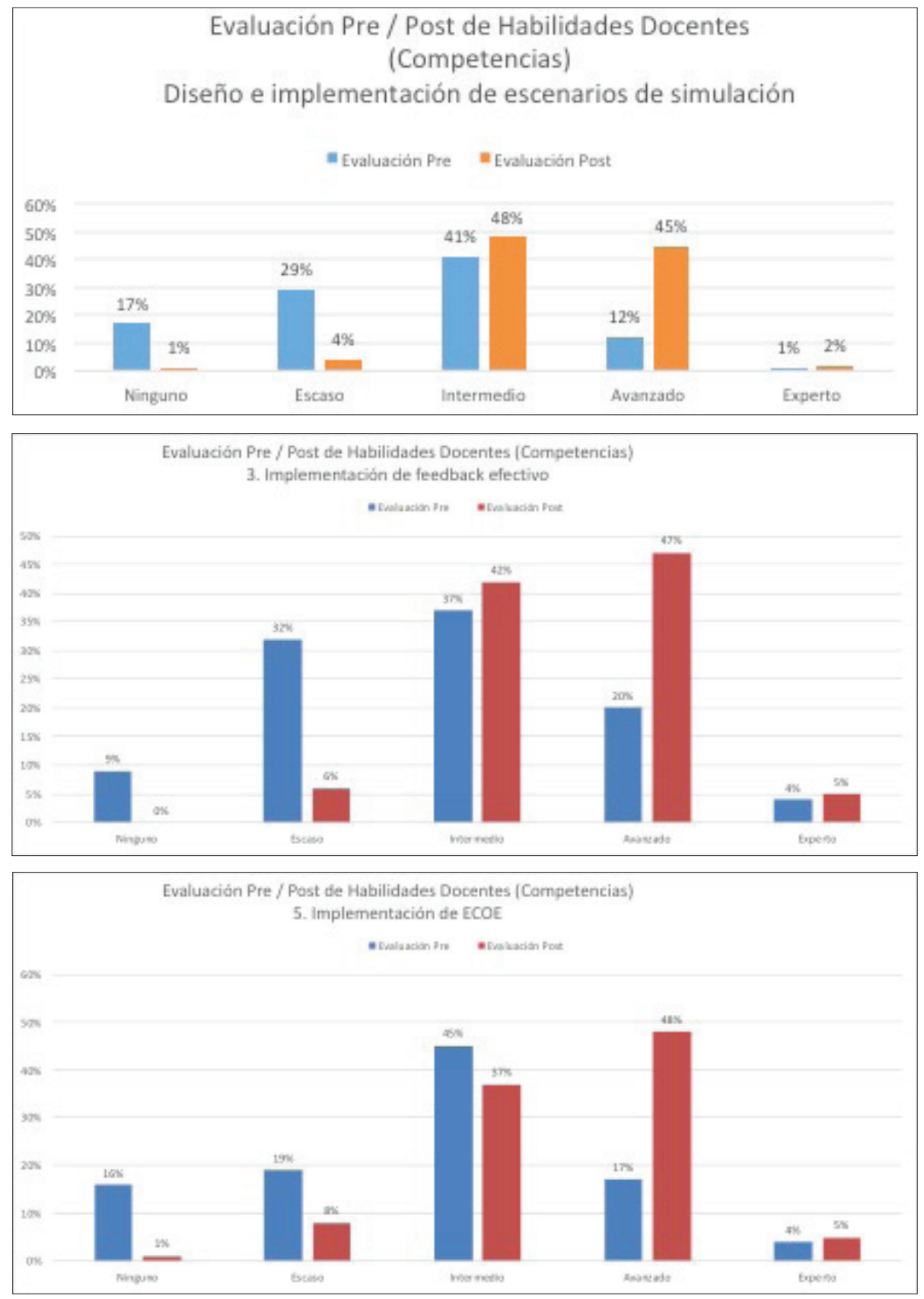

\section{ARS MEDICA Revista de Ciencias Médicas}




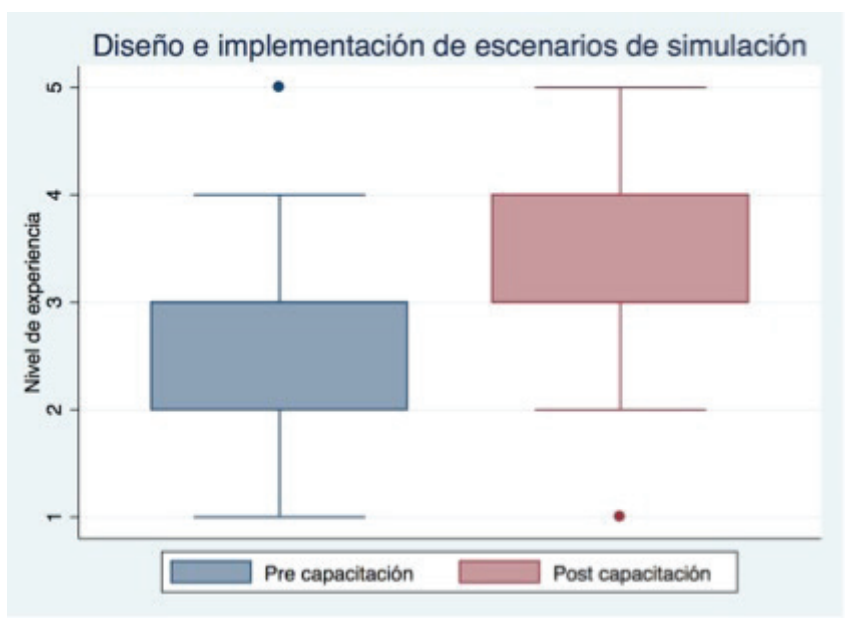

Conclusión: La capacitación docente no solo se relaciona con el desarrollo profesional en términos de aumentar las habilidades profesionales de quienes enseñan en la institución, sino que, es un valor institucional porque tiene la capacidad de movilizar todos sus recursos y equipos en una actualización continua y en la innovación de la enseñanza.Mejora el vínculo entre los docentes y su percepción y motivación en la carrera academica.

\section{Evaluación de necesidades para la implementación de un módulo de enseñanza de ética clínica como parte de los roles canmeds profesional y comunicador en un programa de residencia de neonatología}

Escalante María (1) Fabres Jorge (1)

(1) Departamento de Neonatología. División de Pediatría. Facultad de Medicina. Pontificia Universidad Católica de Chile, Santiago, Chile

Introducción: La ética es un componente crítico de los currículos en la enseñanza de postgrado y la necesidad de un entrenamiento formal en esta área es reconocida por los residentes. El modelo CanMEDS reconoce que el aprendizaje del razonamiento ético no puede ser un objetivo aislado, sino que es parte de las competencias a desarrollar en los roles Profesional y Comunicador. En la especialidad de Neonatología, esto es crucial, debido al enfrentamiento diario de temas como pronóstico incierto, tecnología avanzada para soporte vital y altas expectativas de los padres. Publicaciones señalan que se logra mayor motivación y compromiso cuando su enseñanza ser realiza aplicada al contexto específico de los residentes y del programa, por lo que se debe identificar las necesidades locales para la implementación de cualquier iniciativa. El objetivo es comunicar el resultado de la evaluación de necesidades para la implementación de un módulo de enseñanza de ética clínica desde tres miradas: residentes, egresados y académicos del programa.

Métodos: Se diseñan encuestas para residentes, egresados y académicos del Programa de Neonatología de la Pontifica Universidad Católica de Chile, de acuerdo con los aspectos sobre la enseñanza ética en esta especialidad reportados en la literatura. Se sondea cómo se enseña ética actualmente y las características de un módulo futuro en cuanto a temas a abordar, metodologías, tiempo dedicado, evaluación y participantes.

Resultados: Se envían las encuestas a la totalidad de residentes, egresados y académicos del programa. La recuperación de encuestas fue $30 \%$ para residentes y $50 \%$ para egresados y académicos. La totalidad de los residentes señala que sólo ocasionalmente se abordan aspectos éticos en su feedback de rotación. El 25\% de los egresados declara que su formación en ética durante el programa le permite un desempeño adecuado actualmente. El $40 \%$ de los académicos declara tener entrenamiento formal en ética clínica. Los temas a incluir en un programa futuro con más consenso son: consideraciones éticas en el proceso de toma de decisiones clínicas, entrenamiento en habilidades comunicacionales para la entrega de noticias difíciles y acompañamiento del paciente en proceso de muerte y su familia. Las metodologías propuestas son la discusión de casos en grupo pequeño y presentación en visitas y reuniones clínicas. La estimación del tiempo destinado al aprendizaje de ética durante la residencia fue menos de 5 horas semanales para todos los grupos.

Conclusión: Se detecta la necesidad de sistematizar la enseñanza ética en este programa. La menor tasa de respuesta se dio en el grupo de residentes. Algunos académicos cuentan con entrenamiento formal en ética. Existe consenso sobre los temas mínimos a incluir y sobre la metodología a utilizar, apuntando a un enfoque práctico, con énfasis en habilidades comunicacionales. Sin embargo, el entrenamiento de éstas requiere un enfrentamiento transprofesional, de acuerdo con lo reportado en la literatura, lo cual es un desafío futuro para el programa. 


\section{Implementación de Podcasts como metodología de enseñanza para la Residencia de Psiquiatría Infantojuvenil de la Pontificia Universidad Católica de Chile (PUC): Evaluación de Necesidades Específicas}

\author{
Cortés Camila (1), Valdés Francisca (2), Pacheco Bernardo (3), \\ Inzunza Carla (1), Bedregal Paula (2), Triviño Ximena (3)
}

(1)Departamento de Psiquiatría, (2)Departamento de Salud Pública, (3)Departamento de Educación Médica, Escuela de Medicina, Pontificia Universidad católica de Chile, Santiago, Chile

Introducción: La formación de recursos humanos en salud es un aspecto primordial para la OMS; las metodologías efectivas están basadas en los principios de educación de adultos. La actual generación de residentes utiliza continuamente la tecnología y la tendencia internacional la integra a los currículums educativos; proponemos utilizar podcasts (episodios de audio/video difundidos por internet) como metodología de enseñanza. El objetivo del trabajo es evaluar las necesidades de aprendizaje de los residentes y su disposición al uso de podcast como metodología de enseñanza.

Método: Estudio descriptivo, transversal, de carácter cuantitativo, queexplora necesidades dirigidas de los residentes mediante un cuestionario en línea.

Resultados: Los residentes utilizan principalmente medios de texto para complementar su aprendizaje (83\%), mayormente por motivación propia. En general, desean incorporar a futuro un mayor uso de medios de audio $(66,7 \%)$ y video $(50 \%)$; en particular, videos de entrevistas a un experto sobre un tema seleccionado (75\%). Refieren falta de costumbre en acceder a contenidos de la especialidad vía podcast (58,3\%). Demuestran gran interés en incluir temas de psicopatología en un eventual podcast diseñado para le especialidad, donde 5 temas obtienen la votación máxima (TEPT, EQZ,TDP, TEA y TCA). El 100\% valora especialmente que se incluyan recomendaciones para el enfrentamiento de pacientes. La mayoría (66.7\%) optó por un formato de video de entre 15 y 30 minutos de duración, que fuera evaluado con pruebas de selección múltiple pre y post - podcast (50\%). La mitad de los residentes quiere participar como entrevistador en la elaboración de un podcast para la residencia.

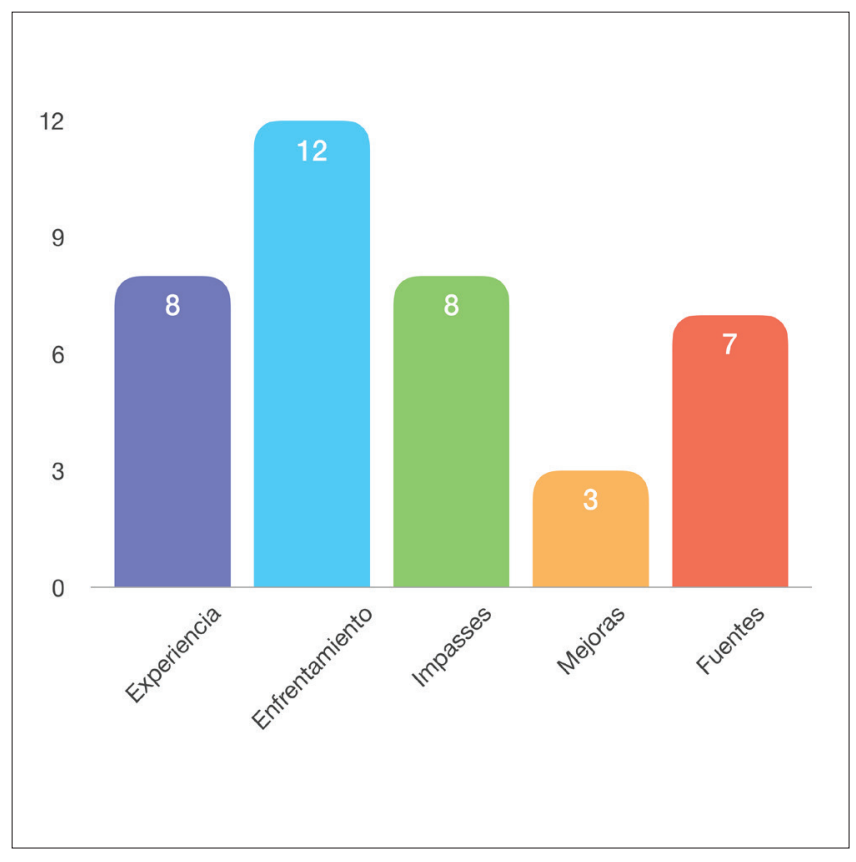

Figura 1. Elementos que debería incluir un podcast de la especialidad.

Eje Y: No de votos. Eje X: Elementos que debería incluir un podcast. Experiencia: Una conversación con un experto sobre su experiencia en el tema seleccionado. Enfrentamiento: Recomendaciones de un experto para enfrentar pacientes del tema seleccionado. Impasses: Impasses a los cuales se haya enfrentado un experto y forma en que los resolvió. Mejoras: Opinión de un experto respecto de qué faltaría por hacer para facilitar el aprendizaje del contenido seleccionado en el programa de residencia. Fuentes: Fuentes de consulta de un experto respecto del tema seleccionado.

Conclusión: La relevancia del presente trabajo es que caracteriza el uso de tecnologías de la información de los residentes de psiquiatría del niño y del adolescente de la UC y sus necesidades en cuanto a recursos electrónicos como metodología de enseñanza; y que permite, en base a nuestros resultados, diseñar dos episodios de entrevista a expertos en formato podcast a partir de los temas seleccionados en conjunto con los residentes, considerando sus preferencias de contenidos y formato e incorporándolos en su elaboración. Su implementación y evaluación, actualmente en curso, serán objeto de una investigación futura. 


\section{Implementación de un taller de elaboración de preguntas estructuradas para evaluación de residentes de Pediatría: una iniciativa para el desarrollo docente.}

\author{
Escalante María (1), Abarca Katia (1), Lizama Macarena (1), Luco \\ Matías (1), Medina Mariana (1), Karzulovic Lorena (1)
}

(1) División de Pediatría. Facultad de Medicina. Pontificia Universidad Católica de Chile, Santiago, Chile.

Introducción: La profesionalización de la docencia en Educación Médica considera la constante capacitación de los docentes para la adquisición de competencias para un mejor desempeño en su labor. Dentro de los 12 roles del docente clínico identificados por Harden y Crosby, se encuentra el rol de evaluador del aprendizaje. A nivel de postgrado y en relación con un currículo basado en competencias, existen instrumentos de evaluación que son más adecuados según el tipo de competencia a evaluar. En el modelo CanMEDS, una de las opciones para la evaluación del rol Médico Experto son las pruebas escritas con preguntas estructuradas. El principal problema se genera en las preguntas de opción múltiple, donde una de las desventajas es la dificultad para escribir buenas preguntas, lo que puede llevar a un stock crítico de las mismas dentro de un programa de residencia. El objetivo es comunicar la experiencia de implementación de un taller de elaboración de preguntas estructuradas para la evaluación de residentes de Pediatría como una experiencia de desarrollo docente en el programa.

Métodos: Se planifica una jornada de 3 horas de capacitación para docentes del programa de residencia de Pediatría de la Pontificia Universidad Católica, cuyos principales objetivos fueron: Identificar los elementos de las preguntas de pruebas escritas con un diseño adecuado y elaborar preguntas para pruebas escritas. Se convocó a los encargados de cada rotación para fomentar la participación de todas las especialidades pediátricas. La estructura del taller fue una clase de 15 minutos donde se abordaron los principales puntos teóricos del tema. Posteriormente, se desarrolló un trabajo colaborativo entre los docentes de las diferentes rotaciones para la revisión y elaboración de nuevas preguntas de forma supervisada. Posterior al taller, se envió a los participantes una encuesta para evaluar el taller y necesidades de formación en educación.

Resultados: Asistieron un total de 18 docentes, con buena representatividad de las diferentes rotaciones. Se recuperó un 55\% de encuestas. El 50\% de los asistentes se desempeña como docente por más de 10 años. Los aspectos más valorados del taller fueron el tener un tiempo protegido para mejorar sus evaluaciones y el trabajo colaborativo con otros docentes. El aspecto por mejorar fue destinar más tiempo para esta capacitación. La principal dificultad reconocida frente a las preguntas de opción múltiple es la construcción de alternativas similares en longitud. La totalidad de los docentes participaría en un nuevo taller de desarrollo docente, con énfasis en otros instrumentos de evaluación. El $75 \%$ de los docentes declara tener formación en Educación Médica a través de diplomado.

Conclusión: Iniciativas de desarrollo docente dentro del programa de Residencia de Pediatría son factibles, con una adecuada participación de académicos. Aunque la mayoría de los participantes contaba con formación docente, todos los académicos manifiestan interés para asistir a este tipo de jornadas. Esto se transforma en un impulso de iniciativas de desarrollo docente más sistematizadas al interior del programa.

\section{Perfil y elecciones de los postulantes a la residencia de Medicina General y/o Familiar del Examen Único Médico desde 2012 a 2018}

Dursi Carolina (1), L`Hopital Cintia (1), Ladenheim Roberta (1), da Cruz Ferreira Heloisa (2), Citate Julieta (1), Duré María Isabel (3), García Marcelo (1)

(1)Dirección Nacional de Capital Humano, Secretaría de Gobierno de SaludMinisterio de Salud y Desarrollo Social. Buenos Aires, Argentina. (2)Dirección Nacional de Epidemiología y Análisis de Situación de Salud, Secretaría de Gobierno de Salud- Ministerio de Salud y Desarrollo Social. Buenos Aires, Argentina. (3)Secretaría de Gobierno de Salud- Ministerio de Salud y Desarrollo Social. Buenos Aires, Argentina.

Introducción: En los últimos años, Argentina ha ampliado la oferta de formación en Medicina General y/o Familiar, sin que haya redundado en un crecimiento de la cobertura de dichos cargos por parte de los postulantes. La especialidad se encuentra en el puesto 37 de preferencia en la elección de residencias, considerando la relación médicos que rindieron examen/cargo. Asimismo, estudios anteriores muestran que una gran proporción de estos especialistas realizan subespecialidades luego de la formación.

El objetivo de este trabajo es describir el perfil de los postulantes a la residencia de Medicina General y/o Familiar de los cargos concursados en el Examen Único (EU) de Ingreso a Residencias Médicas, en todas sus ediciones (2012 a 2018), y analizar las razones de elección de la especialidad y su proyección laboral futura. 
Métodos: Estudio descriptivo de corte transversal. La fuente de datos fue la base de postulantes al Examen Único Médico (EU), que consta de 2283 registros de postulantes a la especialidad de Medicina General y/o Familiar (entre 2012 y 2018). Se relevaron las siguientes variables: sexo, nacionalidad, lugar de residencia, institución de formación de grado, estado civil, promedio de la carrera, lugar de concurso, desempeño en el examen y adjudicación de cargos. Asimismo analizo una encuesta electrónica autoadministrada completada durante la preinscripción al EU, sobre los condicionamientos y preferencias para la elección de la especialidad, la cual fue completada por 1967 postulantes.

Resultados: De los 2283 registros, el $74 \%$ de los postulantes son mujeres, frente al $62 \%$ en el universo de todas las especialidades. El $97,4 \%$ son argentinas/os. El $85,2 \%$ es egresado/a de una universidad pública nacional, el $11 \%$ de una privada y el 3,7\% de una universidad extranjera. En relación con el estado civil, el $88 \%$ son solteros/as frente a un 10,8\% de casados/as. Considerado globalmente, de los 2283 postulantes, solo 1009 adjudicaron un cargo (44\%) a pesar de quedar plazas vacantes.

Entre los motivos principales para la elección de la especialidad encontramos en primer lugar el compromiso social y comunitario y la vocación de trabajo en terreno, y en segundo lugar la compatibilidad con su expectativa de distribución del tiempo entre trabajo, familia y recreación.

En relación a las expectativas futuras, el 53,6\% de los postulantes preferiría trabajar en el subsector público de salud, mientras que al 39,8 le resulta indistinto. En los próximos 8 años, casi el $65 \%$ se imagina trabajando en la especialidad, el $17,6 \%$ se ve realizando una subespecialidad, y solo un $1,8 \%$ se visualiza en cargos de gestión.

Conclusión: El análisis muestra que las preferencias y expectativas antes de iniciar la formación como residentes (ejercer la especialidad y en el ámbito público preferentemente) no se corresponden con el desempeño profesional que efectivamente desarrollan luego de la misma, como se puede ver en investigaciones anteriores sobre la inserción profesional de las y los médicos generalistas y/o de familia.

\section{Elaboración de un instrumento para evaluar profesionalismos en residentes de Medicina Familiar en la Universidad de Concepción}

Alvarado-Figueroa Débora (1), Bustos-Guíñez Andrea (2), PérezWilson Patricia (2), Rico-Soto Felipe (2), Villaseca-Silva Patricia (2)

(1)Departamento de Educación Médica, (2)Programa de Salud y Medicina Familiar, Universidad de Concepción, Concepción, Chile.
Introducción: La evaluación de múltiple fuente o evaluación en $360^{\circ}$ es un tipo de evaluación basada en el lugar de trabajo, que permite evaluar el desempeño real. En el ámbito de la educación médica se ha usado con éxito en pre y postgrado. Consiste en una recopilación sistemática de datos de desempeño durante un periodo en particular, y la entrega de retroalimentación para un estudiante, para algún ámbito en particular, medido mediante cuestionarios estructurados completados por personas involucradas en el quehacer del/la evaluado/a. Por otra parte, el profesionalismo resulta ser un ámbito complejo de evaluar, existiendo evidencia de la utilidad de este tipo de herramientas. Por lo anterior, no existiendo instrumentos de este tipo orientados a evaluar el profesionalismo en este programa de especialización, se decide construirlo participativamente, considerando a las partes involucradas.

Métodos: En junio de 2016 se inicia el proceso de construcción del instrumento con 25 Médicos de Familia docentes en forma presencial, quienes mediante métodos participativos, proponen una serie de conductas profesionales que los residentes deberían evidenciar. Estas fueron agrupadas en las siguientes categorías: Relación con usuarios y familias-Relación con comunidad-Trabajo en equipo-Gestión-Relación con sí mismo-Adaptación a normas institucionales.

Posteriormente, a través de encuestas aplicadas directamente o vía correo electrónico, se solicitó a usuarios, integrantes del equipo de salud, directivos y dirigentes comunitarios, de los distintos territorios en que se desarrolla este programa formativo, que propusieran indicadores de profesionalismo para residentes de medicina familiar. Una vez consolidados estos indicadores, se confeccionó un instrumento preliminar constituido por 74 indicadores, el que fue aplicado en septiembre de 2016 a residentes de primer año, generando retroalimentación y compromisos de mejora de su desempeño. Adicionalmente, los residentes respondieron un cuestionario de evaluación de este proceso. Con estos insumos se reestructuró el instrumento.

Para realizar validez de contenido, el 2017 el cuestionario se envía a evaluación por jueces, solicitando aportes de expertos tanto del ámbito académico como no académico.

Resultados: Se recibió la opinión experta de 6 usuarias/os de los Cesfam que son atendidas por Médicos de Familia, 3 expertos en educación médica, 6 docentes del Programa de Especialización (médicos de familia y otros profesionales del staff). Con estos insumos se mejoraron indicadores y se construyeron 3 cuestionarios, destinados el primero a equipo de salud, pares y autoevaluación; el segundo a usuarias/os, y el tercero a directivos. Con éstos se evalúa actualmente a los residentes de este programa formativo. 
Conclusión: La experiencia participativa de construcción de este instrumento ha permitido considerar la opinión de agentes clave para la formación de médicos de familia (usuarias/os, equipo de salud, directivos) en la evaluación del desempeño del residente, además de construir un perfil de su profesionalismo, por estos mismos agentes. Junto con lo anterior, contar con un instrumento de evaluación auténtica de desempeño, que permite el aprendizaje y mejora continua del profesionalismo en los futuros médicos de familia. Este puede llegar a ser un aporte a otros programas en la medida de que se avance en evidencias de validez y confiabilidad a través de posteriores estudios.

\section{Profundizando la Experiencia Clínica en Procesos Humanos de Ayuda en Medicina}

Cordero B., Rodrigo (1), Pérez-Wilson Patricia (1), Pedraza L., Marcela (1)

(1) Universidad de Concepción, Concepción, Chile.

Introducción: La relación de ayuda se ve influida por una serie de características del usuario, del tratante y de la interacción de ambos. En el Programa de Especialización en Medicina Familiar UdeC se implementó desde el año 2017 la asignatura "Aproximación al rol del terapeuta: desarrollo de herramientas y habilidades para los procesos humanos de ayuda" cuyo propósito es aproximar a los residentes a la experiencia del rol del terapeuta, facilitando el desarrollo de herramientas que permitan alcanzar un nivel de intervención familiar en el nivel 4 (Doherty y Baird), para el trabajo con personas, parejas y familias. Actualmente, existe evidencia suficiente que permite comprender la importancia de "la persona del tratante" como instrumento relevante en el contacto y vinculación con los usuarios, y en la eficacia de los procesos de ayuda. Es por ello, que uno de los aspectos fundamentales de la asignatura es la autoobservación reflexiva de "la persona del médico" respecto de los recursos propios y dificultades para el rol de tratante. Así, como la incorporación de un cuerpo teórico-técnico y entrenamiento que brinden mayor profundidad y alcance a las intervenciones, mejorando la experiencia clínica y los procesos de ayuda. El objetivo de este trabajo ha sido desarrollar habilidades que potencien y profundicen la experiencia clínica otorgando mejores resultados en las relaciones humanas de ayuda.

Métodos: La asignatura tiene una duración de un año y se desarrolla mediante las siguientes actividades: clases en aula con modalidad de educación participativa de adultos, talleres experienciales, reuniones clínicas grupales, y entrevistas individuales de acompañamiento con los residentes orientadas a acompañar la autoexploración de la persona del médico. Los contenidos de la asignatura apuntan a: a) profundidad y alcance de intervenciones, b) desarrollo y efectividad de los procesos de ayuda, c) autoconocimiento d) vínculo médico/usuario, e) acuerdo mutuo de tareas y metas, f) énfasis en los recursos de las personas, parejas y familias, g) entre otros.

Resultados: Los residentes han valorado la asignatura como significativa para su desarrollo de habilidades en la relación de ayuda. $Y$ existen evidencias cualitativas y en algunos casos cuantitativas de: aumento de flexibilidad y apertura frente a los usuarios y sus problemáticas, mayor nivel de vinculación médico/usuario, mayor facilidad en pesquisa y exploración en aspectos biopsicosociales implicados en procesos salud- enfermedad, aumento del impacto de las intervenciones, mayor facilidad en abordaje de pacientes de manejo difícil, disminución de prescripción farmacológica, aumento del autoconocimiento, mayor profundidad y manejo de teorías y técnicas de ayuda, mayor foco en los recursos de los usuarios, parejas y familia, entre otros. Todo esto mejora, a su vez, la adherencia a tratamientos, la resolutividad y la satisfacción usuaria.

Conclusión: Es importante que los médicos en formación presten especial atención a la manera cómo desarrollan la relación con sus usuarios y con sí mismos, entendiendo que estos elementos son centrales en la vinculación, adherencia y efectividad de los tratamientos. Por ello, es necesario que validen y potencien su autoconocimiento y que incorporen un cuerpo teórico-técnico y entrenamiento suficiente que faciliten el desarrollo de herramientas y habilidades que le permitan profundizar y potenciar la relación de ayuda. Siendo el principal aporte al ejercicio profesional del médico, el aumento del repertorio y alcance en sus intervenciones para un tratamiento más integral y efectivo.

\section{Diseño e implementación de un instrumento de evaluación del desempeño para Anatomía Patológica:Mini Pathological Examination Exercise (Mini-PEX).}

Teo Feuerhake (1), Isabel Leiva (2), Sergio González (1), Ximena Triviño (3), Antonieta Solar (1)

(1) Departamento de Anatomía Patológica, (2) Departamento de Enfermedades Respiratorias, Escuela de Medicina, Pontificia Universidad Católica de Chile, (3) Universidad San Sebastián, Santiago, Chile.

Introducción: La calidad y la seguridad asociadas a la atención de salud requieren de la certificación de competencias en médicos especialistas. Modelos de educación médica basada en competencias, como el modelo CanMEDS, tienen por objetivo 
desarrollar y certificar estas competencias en residentes. Estos modelos han hecho énfasis en la evaluación por observación directa en el sitio de trabajo. Por otro lado, la evaluación formativa y el feedback permiten a los residentes desarrollar estas competencias. El objetivo de este proyecto fue diseñar e implementar un instrumento de evaluación por observación directa, basado en el modelo CanMEDS, para la presentación de casos clínicos en microscopios multicabezales en el programa de especialidad en Anatomía Patológica.

Metodología: Se utilizó metodología mixta. Se preseleccionaron competencias CanMEDS que tributasen a la etapa de presentación de casos clínicos. Luego, se seleccionaron aquellas que pudiesen ser evaluadas por observación directa mediante metodología Delphi modificada. Además, se llevaron a cabo sesiones de capacitación docente. El instrumento fue diseñado y se realizó su aplicación piloto. La aplicación fue evaluada y los resultados fueron analizados.

Resultados: Las competencias seleccionadas tributan a los roles de Médico Experto, Comunicador y Profesional del modelo CanMEDS. Las competencias fueron agrupadas y se diseñó un instumento de seis dimensiones que considera la entrega de feedback inmediato. Se confeccionó una escala de calificación cualitativa de siete niveles de logro. El diseño del instrumento asegura validez de contenido para sus resultados. Los resultados y la entrega de feedback con el instrumento fueron bien recibidos y la satisfacción fue alta tanto en residentes como docentes.

Conclusión: Se diseñó un instrumento de evaluación por observación directa basado en el modelo CanMEDS que considera la entrega de feedback inmediato. El desarrollo de un instrumento de evaluación por observación directa es una innovación en Anatomía Patológica y la validez de contenido de sus resultados se encuentra asegurada por su diseño. El instrumento ayuda a estructurar la presentación de casos clínicos y resalta la importancia de competencias tanto cognitivas como actitudinales. El consenso de expertos limitado a un contexto local es la mayor limitación de este proyecto.

\section{Cómo enseñar procedimientos simulados para la adquisición de habilidades técnicas. Experiencia inicial de un curso para residentes}

Jarry Cristián (1), Abbott Eduardo (2), Letelier Luz María (2), Poblete Rodrigo (2), Zamorano Elga (1), Varas, Julián (1)

(1)Centro de Simulación y Cirugía Experimental, (2)Departamento de Medicina Interna, Pontificia Universidad Católica de Chile, Santiago, Chile.
Introducción: La simulación de procedimientos en medicina es una herramienta aceptada para enseñar y entrenar a alumnos en distintos niveles de formación. Históricamente, los residentes han asumido un rol docente respecto de alumnos de pregrado y de pares con menor formación, en especial en la supervisión de procedimientos. Se espera de ellos que desarrollen la capacidad de enseñar a otros (scholar) y, si bien existen cursos formales en algunas malla de especialidad que permiten cumplir con este objetivo no se han desarrollado cursos que permitan perfeccionar la docencia mediante simulación. El presente trabajo busca describir y evaluar la percepción sobre un curso transversal de formación de tutores en procedimientos simulados, orientado a residentes.

Métodos: Estudio descriptivo. Durante los años 2017 y 2018, se impartió el curso "Como enseñar procedimientos simulados para la adquisición de habilidades técnicas" en el Centro de Simulación de la Universidad Católica, a residentes de diversas especialidades. Su objetivo consistió en empoderar a residentes respecto a la realización de procedimientos y su enseñanza. El curso se estructuró en base a un módulo teórico y uno práctico. El primero consistió en 4 sesiones presenciales donde se revisaron conceptos de educación médica, teoría del aprendizaje y simulación, además de la evidencia disponible respecto a estos temas. El segundo, consistió en una sesión de entrenamiento para conocer modelos simulados y 2 sesiones prácticas, donde se desempeñaron como tutores de talleres de Simulación en Paracentesis y Toracocentesis orientados a pregrado. Se envió encuesta a residentes para evaluar su percepción sobre la utilidad del curso en su formación como docentes de procedimientos.

Resultados: Un total de 25 residentes que completaron el curso respondieron la encuesta. El $40 \%(n=10)$ correspondió a residentes de especialidades quirúrgicas, el $20 \%(n=5)$ a residentes del área de medicina interna y el $40 \%(n=10)$ a residentes de otras especialidades. La moda de años de residencia fue primer año $(40 \%, n=10)$. El 52\%(n=13) contaba con experiencia previa de docencia en procedimientos. Respecto a la evaluación del curso, el 100\%(n=25) calificó como relevante o fundamental el aporte en su posterior desempeño como tutores y el $96 \%(n=24)$ declaró acuerdo respecto a que son necesarias instancias de este tipo para formar a quienes se desempeñaran como docentes de procedimientos. Respecto a las herramientas entregadas, se destacó el conocimiento respecto a: Pautas de evaluación $(80 \%, n=20)$, entrega de feedback $(80 \%$, $\mathrm{n}=20)$ y bases teóricas de la simulación $52 \%(\mathrm{n}=13)$. La instancia fue evaluada positivamente, obteniendo una calificación 6 o 7 en el $96 \%(n=24)$ de los casos, usando escala habitual (notas 1 a 7$)$.

Conclusión: Cursos abreviados en los cuales los residentes adquieran herramientas docentes en procedimientos son evaluados 
positivamente y reconocidos como relevantes para obtener un buen desempeño como tutores de alumnos de pregrado. Estas instancias no solo permiten obtener docentes calificados, sino que empoderan a los residentes a ser críticos en la realización de sus propios procedimientos y de sus pares.

\section{Cambios implementados en los programas de especialidad y subespecialidad médica luego de conocer los resultados de la Encuesta Anual de la Docencia de Postgrado.}

Silva María Paz (1)

(1) Pontificia Universidad Católica de Chile, Santiago, Chile

Introducción: Anualmente, la Dirección de Postgrado de la Facultad de Medicina de la Pontificia Universidad Católica de Chile aplica una Encuesta a los residentes de los distintos programas de especialidad y subespecialidad, que evalúa la docencia, el clima educacional al interior de los programas, síndrome de burnout, cuestionario de maltrato, entre otros. El objetivo de este estudio es conocer el impacto de los resultados de esta encuesta en los programas específicos en términos de cambios implementados o a implementar, luego de recibir feedback de los resultados generales obtenidos en ella.

Métodos: Como estrategia de recolección de datos, se utilizó una encuesta estructurada de metodología mixta (cuali-cuanti) en formato digital, que fue enviada a los jefes de cada programa de especialidad o subespecialidad, para que evaluaran el proceso de la Encuesta Anual de la Docencia, su relevancia y repercusiones al interior de sus programas.

Resultados: El 77\% ( $n=20)$ de los jefes de programa encuestados señala haber hecho o que hará cambios en su programa luego de recibir los resultados de la encuesta. Los cambios más frecuentemente realizados fueron hechos en: metodologías docentes (67\%), prácticas docentes (71\%) y en ambiente educacional (86\%). El 69\% señala además que hará cambios en los sistemas de evaluación.

Conclusión: Este estudio permite hacer seguimiento de la Encuesta de la Docencia de Postgrado y destaca su importancia ante los jefes de cada programa, incentivando la implementación de mejoras basadas en las opiniones vertidas por los residentes acerca de su programa de formación.

\section{Uso de TICs en Educación Médica. Experiencia NIRVE en Chile}

Rocha Diego (1), Idiáquez Juan (1), Acosta Ignacio (1), Gros Priti (2)

(1) Servicio de Neurología, Departamento de Neurología y Psiquiatría, Facultad de Medicina, Clínica Alemana. Santiago, Chile, (2)Division of Neurology, University of Toronto. Ontario, Canada.

Introducción: La educación e investigación médica están en continuo cambio. El uso de las tecnologías de la información y la comunicación (TIC), han impulsado el desarrollo científico-tecnológico, siendo una herramienta de apoyo para el desarrollo de los procesos de enseñanza - aprendizaje, así como han permitido la creación de nuevos escenarios docentes y el empleo de nuevos medios de enseñanza. La internacionalización es reconocida como una de las acciones con mayor impacto en la calidad académica de las instituciones de educación superior. El uso de videoconferencia es una forma comprobada de complementar la educación médica, permitiendo el aprendizaje y la interacción entre pares a nivel mundial. The Neurology International Residents Videoconference and Exchange (NIRVE) es una iniciativa dirigida por residentes, fundada en 2009 por la División de Neurología de la Universidad de Toronto para promover la educación a través de la colaboración internacional. NIRVE fue diseñado para desarrollar habilidades de liderazgo y crear oportunidades para que los residentes de todos los niveles participen en la educación médica, aumenten la conciencia de los conceptos de salud global en neurología, refuercen la colaboración internacional y nacional entre los residentes de neurología, y actuar como puerta de entrada para organizar intercambios. Los participantes internacionales son: Hospital St Michael's (Toronto, Canada), Hospital Sick Kids (Toronto, Canada), Universidad de Ottawa (Canada), Universidad Federal de São Paulo (Sao Paolo, Brazil), First Saint-Petersburg Pavlov State Medical University (Russia), Bashkir State Medical University (Ufa, Russia) y Universidad del Desarrollo (Santiago, Chile). El objetivo propuesto fue desarrollar un modelo innovador de teleeducación, utilizando plataformas en línea y videoconferencia vía web, que permitan fortalecer los procesos educativos en la formación de residentes de Neurología.

Métodos: Las reuniones médicas se llevan a cabo el primer jueves de cada mes entre septiembre y junio. Comienzan a las 8 am, hora del este, con una presentación de 30 minutos y un "desafío de imagen" de 15 minutos. Enfocado en un diagnóstico radiológico o patológico, acompañado de neuroimágenes. Las reuniones médicas incluyen tiempo suficiente para involucrar a los residentes en discusiones sobre pasos de diagnóstico y manejo terapéutico en los diferentes sitios internacionales. 
Resultados: El programa de postítulo en Neurología de la Universidad del Desarrollo se unió a la iniciativa NIRVE desde el año 2017, desde entonces 8 residentes de neurología, 3 fellow de subespecialidad, 9 neurólogos y 2 neuroradiólogos han asistido a las reuniones médicas de NIRVE. Se designaron dos residentes para promocionar, difundir, y participar en intercambio y reuniones presenciales en la World Federation of Neurology. Se identificaron como dificultades: Programación de horarios ( $n=3)$; barreras idiomáticas $(n=2)$, inestabilidad de la señal web durante la videoconferencia $(n=2)$.

Conclusión: El uso de TICs facilita la gestión del conocimiento, la experiencia NIRVE en Chile ha complementado la educación médica de los residentes de neurología y puede representar una solución sostenible para el aprendizaje entre iguales a nivel mundial.

\section{Research Fundamentals: Instrumentalization of Residentes}

Souza Regina (1), Saito Katya (2), Vieira Ricardo (3), Santos Audry

(1) Nursing, Núcleo de Novos Conhecimentos. (2)Coordinator, Núcleo de Novos Conhecimentos. (3)Nursing, Núcleo de Novos Conhecimentos. (4) Nursing Manager, Núcleo de Novos Conhecimentos. Sirio Libanes Hospital, Sao Paulo, Brazil.

Introduction: In the legal aspect, the Brazilian Constitution of 1988, through article 207, dictates that higher education should guide the formation of its students in the tripod teaching, research and extension ${ }^{1}$. Although undergraduate students in the health field are interested in research, there is a gap in the educational system that does not encourage them to investigate and produce knowledge ${ }^{2}$. Residency is the opportunity to acquire technical, institutional and relational experience, being a path for those seeking a more solid professional formation ${ }^{3}$. It is also an essential moment for the improvement of the practice of evidence-based medicine with a multiprofessional vision. Thus, it contributes to the integration of knowledge and the promotion of a broader, more effective and effective assistance ${ }^{4}$.

So, was observed that the residents presented a difficulty in the construction and consumption of evidence derived from studies, actions were implemented in the institution focused on the purpose of fomenting evidence-based practice. The purpose of the report is to describe how the research fundamentals discipline was developed and implemented.

Method: Experience report on the development of the discipline "Research Fundamentals", held from August to December 2018 for residents of the uniprofessional and multiprofessional programs
(Biomedicine, Nursing, Pharmacy, Medical Physics, Physiotherapy, Nutrition and Psychology) of the first year, with a workload of 32 hours, being 28 hours in person and four hours at a distance.

The competences contemplated in the discipline were planning of scientific research with adequate structure the guiding question of the study, elaboration of careful argumentation and with research proposals grounded, data collection that can be discussed with other professionals for elaboration and the reelaboration of the knowledge and realization of search for judicious literature in electronic databases.

For the development of these competences, active methodologies were used, with the division of the residents into four classes. The content of the course included notions of research, database research, sampling techniques, ethical aspects, literature reviews, observational drawings, intervention research and research submission platforms.

Results: A hundred residents were instrumental, of which 66 answered the sociodemographic profile questionnaire.

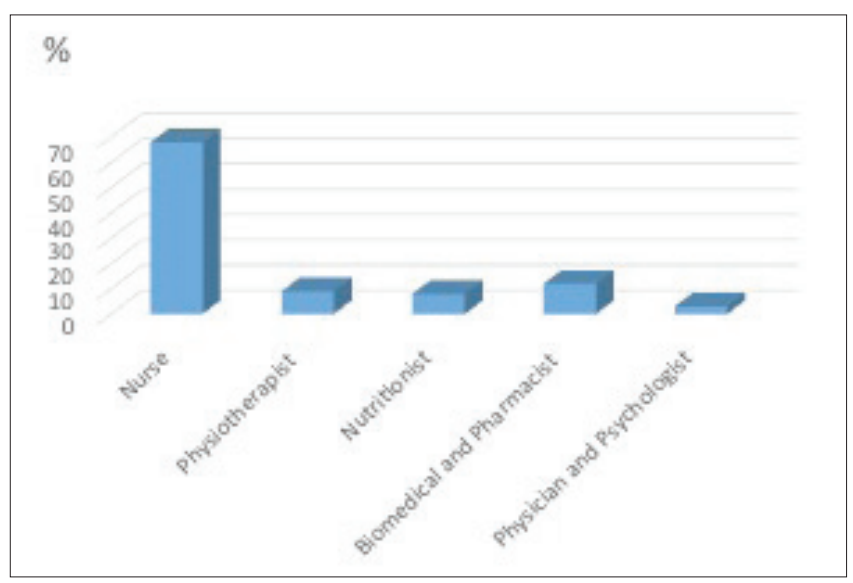

Graph 1. Distribution of the professional categories of the residents. São Paulo, 2018.

Table 1. Distribution of residents according to program. São Paulo, 2018.

\begin{tabular}{lc}
\hline Residence Program & $\%$ \\
\hline Critical Care Patient Care Residency Program & 23 \\
Oncology Patient Care Residency Program & 18 \\
Child Health Care Residency Program & 10.6 \\
Clinical-Surgical Nursing Residency Program & 10.6 \\
Nursing in the Surgical Center and Sterilized Material Center & 10.6 \\
Residency Program & 9 \\
Emergency and Emergency Nursing Residency Program & 7.5 \\
Cardiology Nursing Residency Program & 6 \\
Biomedicine in Diagnostic Imaging Residency Program, & 3 \\
HealthCareManagementandHealthCareNetworks ResidencyProgram & 3.5 \\
\hline
\end{tabular}




\section{Conclusion:}

It is hoped that this discipline may contribute to the development of scientific research and to the implementation of evidence-based practice, which are fundamental aspects for reflection on professional and educational practice and improvement of the quality of care. A greater number of nurses is observed because the number of places for this professional category in the programs is predominant.

\section{Capacidad de Empatía en Docentes de la Facultad de Medicina de la Pontificia Universidad Católica del Ecuador-Quito, desde Octubre del 2017 a Diciembre del 2017}

Carrión Freddy (1), Camacho Gustavo (1), Rodas Irene (1)

\section{(1) Pontificia Universidad Católica del Ecuador}

Introducción: La empatía es un factor primordial en el desarrollo de las relaciones interpersonales para entender los problemas, logros, ilusiones y todos los sentimientos de un individuo. Es por esto que la empatía, en el nivel educativo, juega un papel primordial. Estudiantes que tienen maestros empáticos van a tener un mejor desarrollo y se interesarán por las actividades propuestas. Por esto, la empatía es un punto clave para una correcta relación entre docente y estudiante.

El objetivo fue determinar la capacidad de empatía de los docentes de la Facultad de Medicina de la Pontificia Universidad Católica del Ecuador (PUCE) en los meses de octubre a diciembre del 2017

Métodos: Se realizó un estudio descriptivo mediante encuestas transversales, test de Baron Cohen de forma virtual y física. Un total de 97 docentes respondieron a nuestra encuesta. Se compararon los resultados con variables de edad, género, años de docencia y asignatura impartida.

Resultados: Se observó que el 91,8\% de los docentes encuestados tienen una alta capacidad de empatía, mientras que el 8,2\% tuvo una baja capacidad de empatía. Los docentes más jóvenes poseen mayor capacidad de empatía con respecto a docentes de mayor edad; además, los docentes con menos tiempo de servicio como profesores de la Facultad de Medicina tuvieron mejor capacidad de empatía que los docentes con más años.
Tabla 1. Características generales de la población

\begin{tabular}{|c|c|c|c|}
\hline VARIABLE & & FRECUENCIA & PORCENTAJE \\
\hline \multirow{4}{*}{ EDAD } & 30 a 40 & 21 & 21.6 \\
\hline & 40 a 50 & 30 & 30.9 \\
\hline & 50 a 60 & 32 & 33.0 \\
\hline & $>\mathrm{A} 60$ & 14 & 14.4 \\
\hline \multirow{2}{*}{ SEXO } & MASCULINO & 48 & 49,5 \\
\hline & FEMENINO & 49 & 50,5 \\
\hline \multirow{4}{*}{$\begin{array}{l}\text { TIEMPO DE } \\
\text { DOCENCIA }\end{array}$} & MENOR A 10 & 58 & 59,8 \\
\hline & DE 10 A 20 & 27 & 27,8 \\
\hline & DE 20 A 30 & 10 & 10,3 \\
\hline & MAYOR DE 30 & 2 & 2,1 \\
\hline \multirow{3}{*}{$\begin{array}{l}\text { ESPECIALI- } \\
\text { DAD }\end{array}$} & CLÍNICO & 70 & 72,2 \\
\hline & QUIRÚRGICO & 12 & 12,4 \\
\hline & COMPLEMENTARIA & 15 & 15,5 \\
\hline \multirow{7}{*}{$\begin{array}{l}\text { A S I G N A - } \\
\text { TURA QUE } \\
\text { IMAPRTE } \\
\text { EN LA FA- } \\
\text { CULTAD }\end{array}$} & $A B P$ & 34 & 35,1 \\
\hline & $\mathrm{PH}$ & 9 & 9,3 \\
\hline & APS & 9 & 9,3 \\
\hline & MORFOFUNCION & 35 & 36,1 \\
\hline & ADSAN & 5 & 5,2 \\
\hline & PSICOSOCIAL & 4 & 4,1 \\
\hline & OTRO & 1 & 1 \\
\hline \multirow{3}{*}{$\begin{array}{l}\text { TIEMPO DE } \\
\text { DEDICACIÓN }\end{array}$} & TIEMPO COMPLETO & 51 & 52,6 \\
\hline & MEDO TIEMPO & 16 & 16,5 \\
\hline & TIEMPO PARCIAL & 30 & 30,9 \\
\hline \multirow{3}{*}{$\begin{array}{l}\text { LUGAR DE } \\
\text { DESARRO- } \\
\text { LLO DE LA } \\
\text { MATERIA }\end{array}$} & AULA & 71 & 73,2 \\
\hline & HOSPITAL & 15 & 15,5 \\
\hline & COMUNIDAD & 11 & 11,3 \\
\hline
\end{tabular}

Tabla 2. Resultados de empatía

\begin{tabular}{c|c|c}
\hline \multicolumn{3}{c}{ EMPATÍA } \\
\hline EMPATÍA & Frecuencia & Porcentaje \\
\hline BAJA & 8 & 8,2 \\
\hline ALTA & 89 & 91,8 \\
\hline Total & 97 & 100,0 \\
\hline
\end{tabular}


Volumen 44 suplemento 1

Tabla 3: Relación de edad con capacidad de empatía

\begin{tabular}{|c|c|c|c|}
\hline \multirow{2}{*}{ VARIABLE } & \multicolumn{3}{|c|}{ EMAPTÍA } \\
\cline { 2 - 4 } & FRECUENCIA & BAJA & ALTA \\
\hline $\mathbf{3 0}$ a $\mathbf{4 0}$ & 21 & $0,00 \%$ & $100,00 \%$ \\
\hline $\mathbf{4 0}$ a $\mathbf{5 0}$ & 30 & $6,70 \%$ & $93,30 \%$ \\
\hline $\mathbf{5 0}$ a $\mathbf{6 0}$ & 32 & $9,40 \%$ & $90,60 \%$ \\
\hline Mayor a 60 & 14 & $21,40 \%$ & $78,60 \%$ \\
\hline
\end{tabular}

CHI CUADRADO 5,255; P $<0,5$

Tabla 4: Tiempo de docencia en la facultad en relación a la capacidad de empatía

\begin{tabular}{|c|c|c|c|}
\hline \multirow{2}{*}{ VARIABLE } & \multicolumn{3}{|c|}{ EMAPTÍA } \\
\cline { 2 - 4 } & FRECUENCIA & BAJA & ALTA \\
\hline Menor a 10 & 58 & $3,40 \%$ & $96,60 \%$ \\
\hline De 10 a 20 & 26 & $11,10 \%$ & $88,90 \%$ \\
\hline De 20 a 30 & 10 & $20,00 \%$ & $80,00 \%$ \\
\hline Mayor de 30 & 2 & $50,00 \%$ & $50,00 \%$ \\
\hline
\end{tabular}

CHI CUADRADO 8,491; P $<0,05$

Tabla 5: Especialidad del docente en relación a la capacidad de empatía

\begin{tabular}{|c|c|c|c|}
\hline \multirow{2}{*}{ VARIABLE } & \multicolumn{3}{|l|}{ EMAPTÍA } \\
\hline & FRECUENCIA & BAJA & ALTA \\
\hline CLÍNICO & 70 & $7,10 \%$ & $92,90 \%$ \\
\hline QUIRÚRGICO & 12 & $8,30 \%$ & $91,70 \%$ \\
\hline COMPLEMENTARIO & 15 & $13,30 \%$ & $86,70 \%$ \\
\hline
\end{tabular}

CHI CUADRADO 0,626; P >0,05

Tabla 6: Tiempo de dedicación horaria en relación a la capacidad de empatía

\begin{tabular}{|l|l|l|l|}
\hline \multirow{2}{*}{ VARIABLE } & \multicolumn{2}{|l|}{ EMAPTÍA } \\
\cline { 2 - 4 } & FRECUENCIA & BAJA & ALTA \\
\hline TIEMPO COMPLETO & 51 & $9,80 \%$ & $90,20 \%$ \\
\hline MEDIO TIEMPO & 16 & $6,30 \%$ & $93,80 \%$ \\
\hline TIEMPO PARCIAL & 30 & $6,70 \%$ & $93,30 \%$ \\
\hline
\end{tabular}

CHI CUADRADO 0,347; P > 0,05
Tabla 7: Lugar de desarrollo de la materia en relación a la capacidad empatía

\begin{tabular}{|c|c|c|c|}
\hline \multirow{2}{*}{ VARIABLE } & \multicolumn{3}{|c|}{ EMPATÍA } \\
\cline { 2 - 4 } & FRECUENCIA & BAJA & ALTA \\
\hline AULA & 51 & $9,80 \%$ & $90,20 \%$ \\
\hline HOSPITAL & 16 & $6,30 \%$ & $93,80 \%$ \\
\hline COMUNIDAD & 30 & $6,70 \%$ & $93,30 \%$ \\
\hline
\end{tabular}

CHI CUADRADO 0,659; P >0,05

Tabla 8: Asignatura impartida en relación a la capacidad de empatía

\begin{tabular}{|c|c|c|c|}
\hline \multirow{2}{*}{ VARIABLE } & \multicolumn{3}{|c|}{ EMAPTÍA } \\
\cline { 2 - 4 } & FRECUENCIA & BAJA & ALTA \\
\hline ABP & 33 & $8,80 \%$ & $91,20 \%$ \\
\hline PH & 9 & $11,10 \%$ & $88,90 \%$ \\
\hline APS & 9 & $0,00 \%$ & $100,00 \%$ \\
\hline MORFOFUNCIÓN & 35 & $5,70 \%$ & $94,30 \%$ \\
\hline ADSAN & 5 & $0,00 \%$ & $100,00 \%$ \\
\hline PSICOSOCIAL & 4 & $50,00 \%$ & $50,00 \%$ \\
\hline OTRO & 1 & $0,00 \%$ & $100,00 \%$ \\
\hline
\end{tabular}

CHI CUADRADO 10,972; P >0,05

Tabla 9: Sexo en relación a la capacidad de empatía

\begin{tabular}{|c|c|c|c|}
\hline \multirow{2}{*}{ VARIABLE } & \multicolumn{3}{|c|}{ EMAPTÍA } \\
\cline { 2 - 4 } & FRECUENCIA & BAJA & ALTA \\
\hline MASCULINO & 48 & $10,40 \%$ & $89,60 \%$ \\
\hline FEMENINO & 49 & $6,10 \%$ & $93,95 \%$ \\
\hline
\end{tabular}

CHI CUADRADO 0,591; $\mathrm{P}<0,05$

Conclusión: La mayoría de docentes de la facultad de Medicina de la PUCE tienen alta capacidad de empatía. La edad de los docentes tiene una fuerte relación con la capacidad de empatía. 